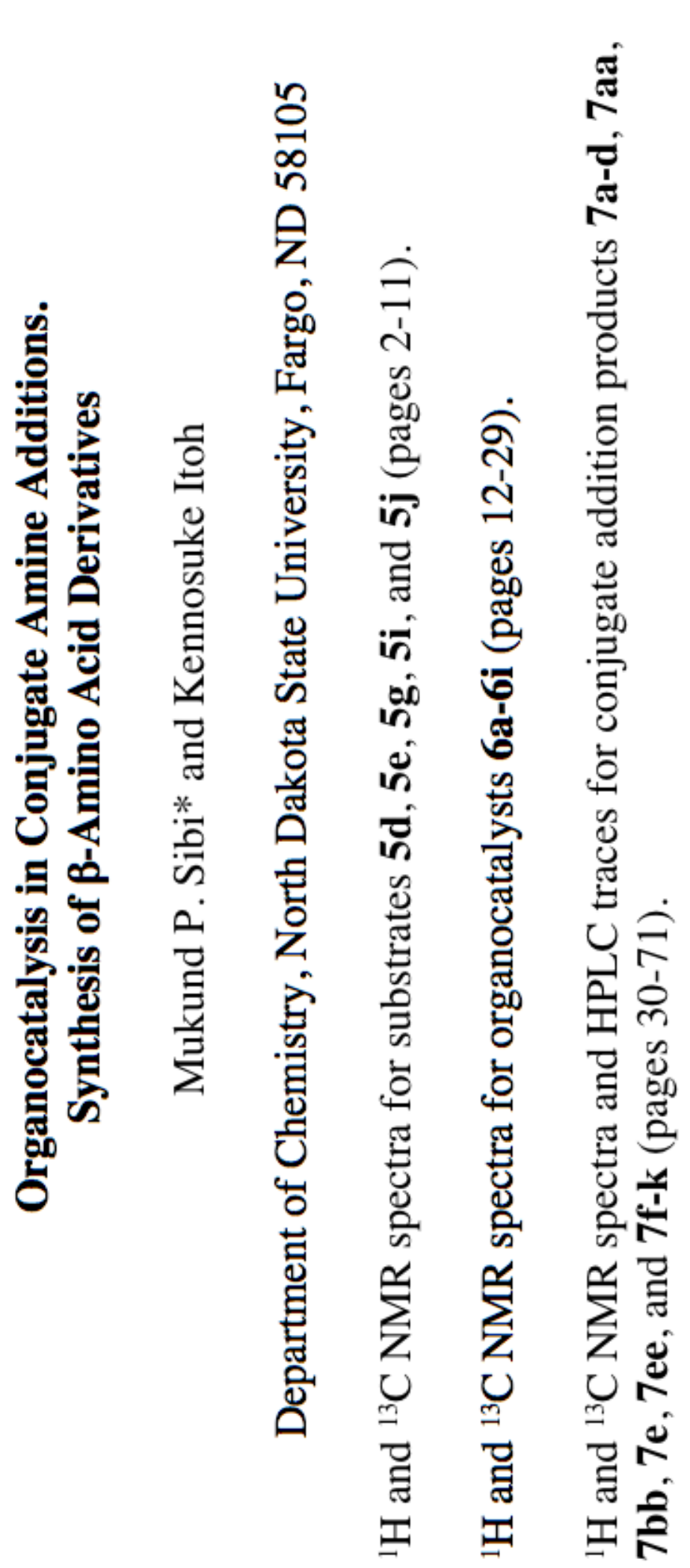




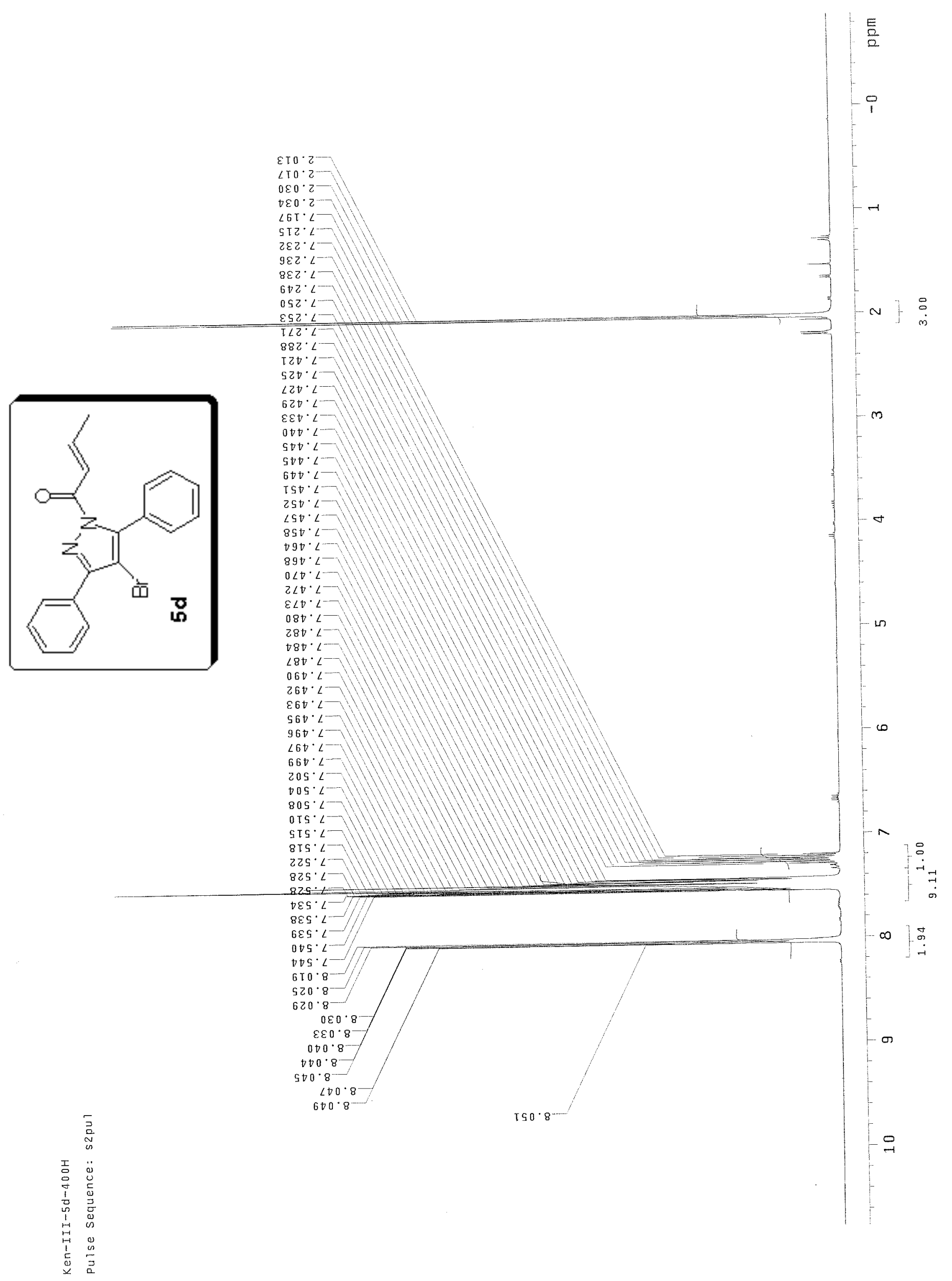




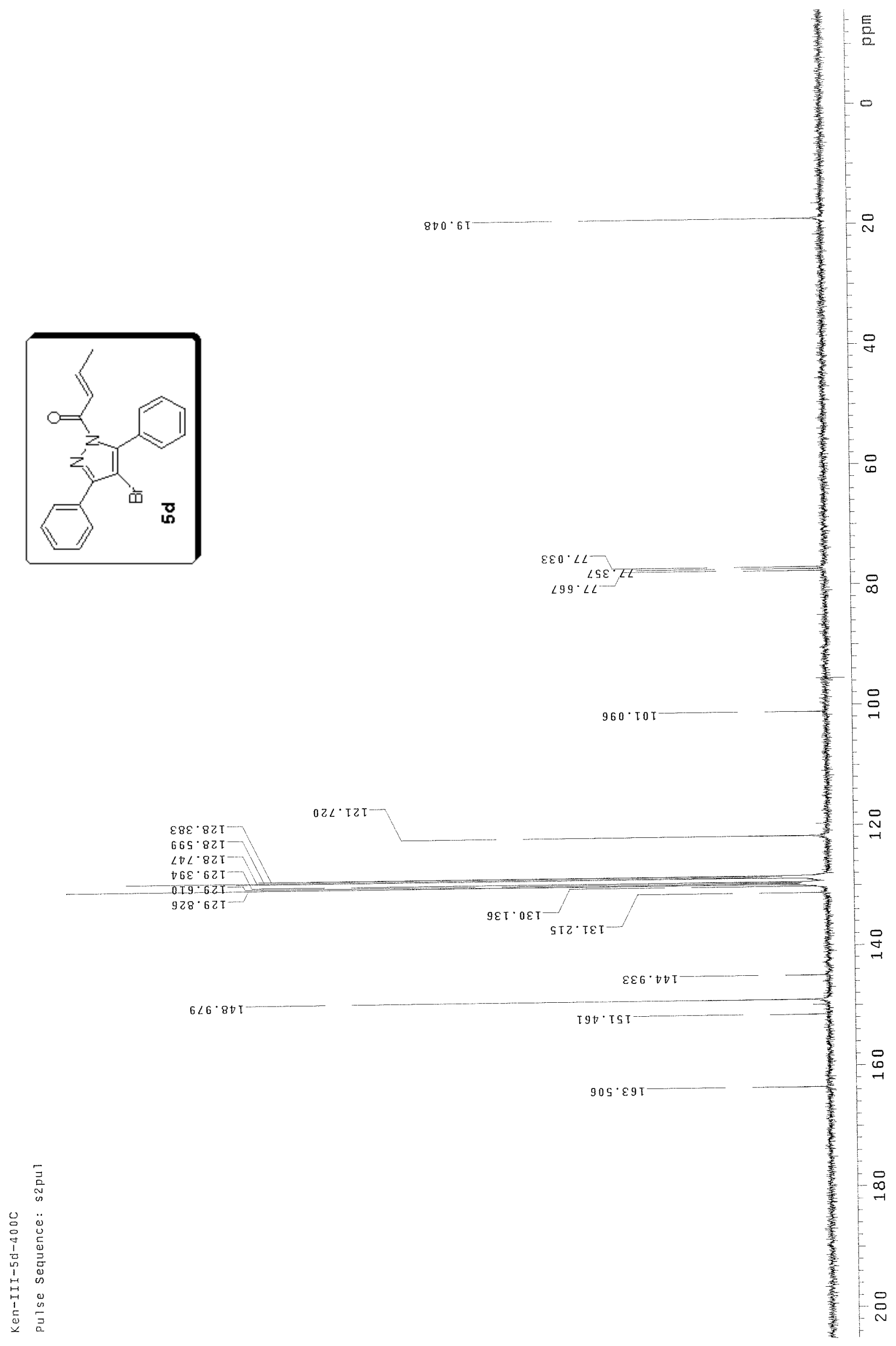




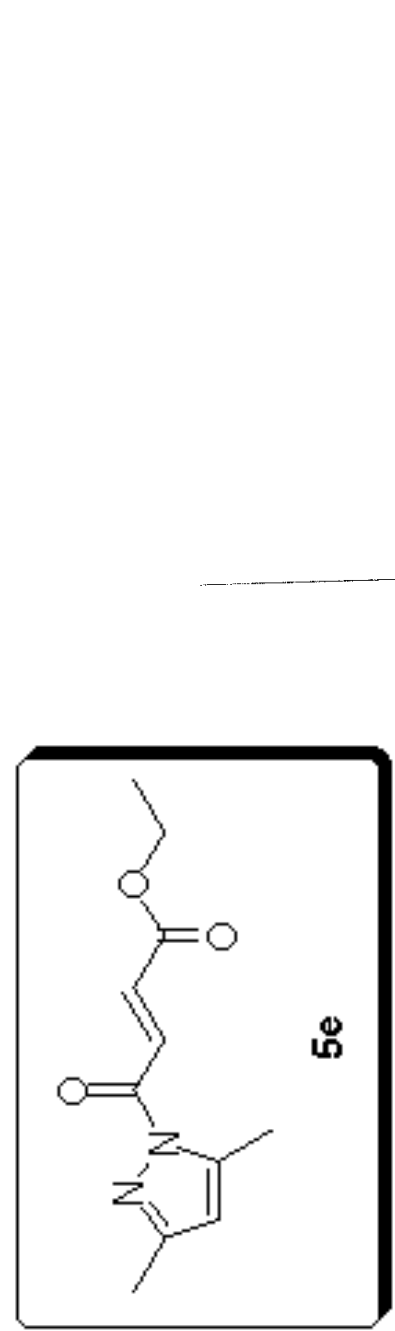

$090 \cdot \frac{2}{290}$
$880 \cdot 2$

$92 z \cdot t$

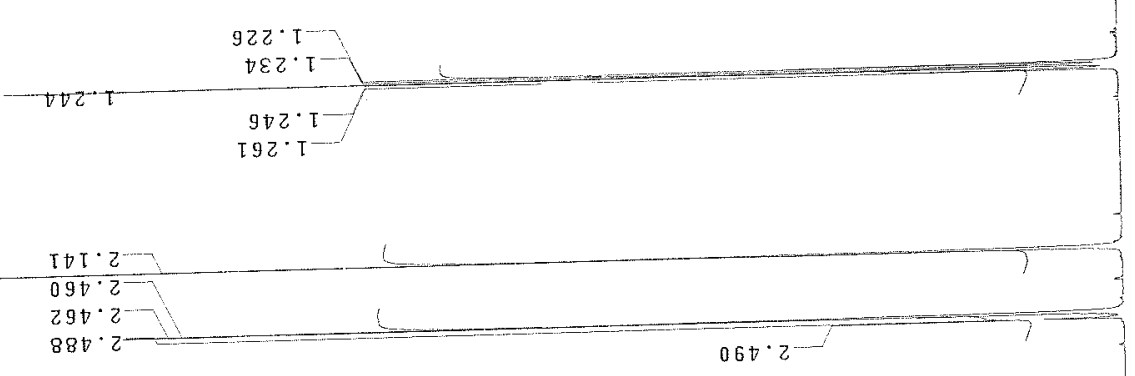

$I_{\infty}^{\infty}$

N

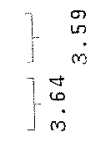

$m$

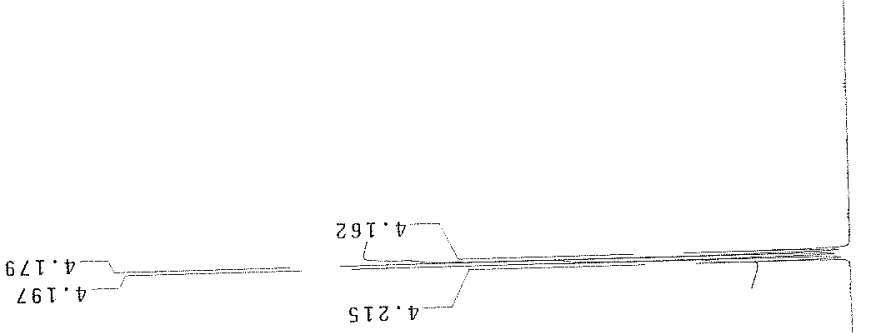

$\leftarrow$

$\stackrel{\infty}{\stackrel{-}{\sim}}$

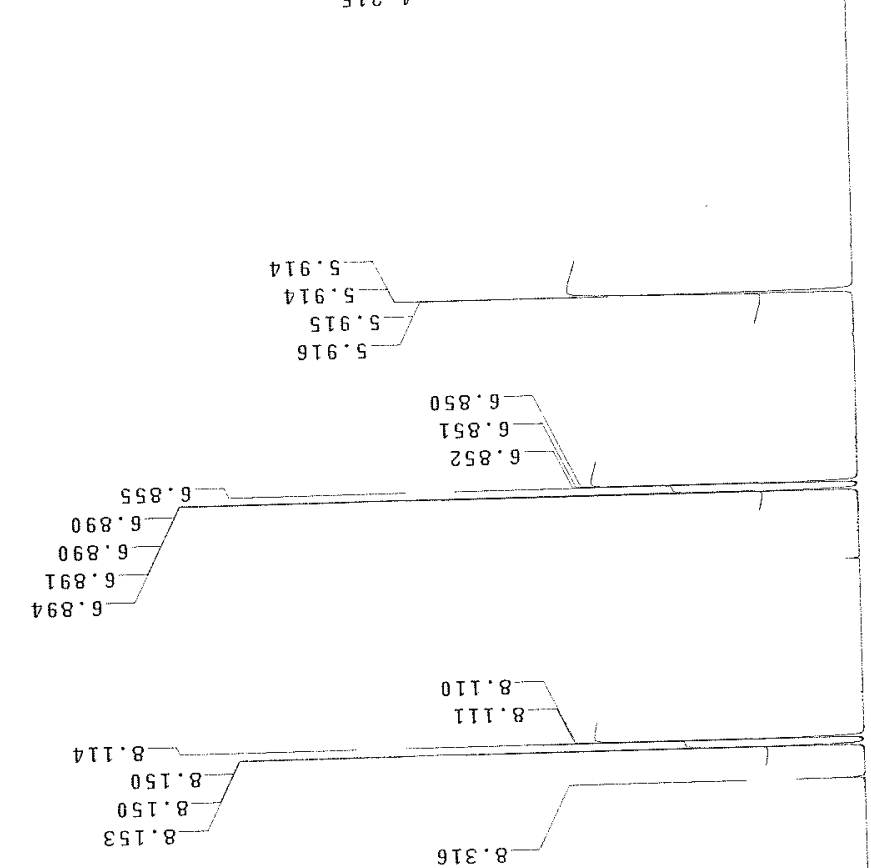

1

$\infty \quad \begin{aligned} & 0 \\ & 0\end{aligned}$

$-\quad \overrightarrow{0}$

渵

5 T. 8

$9 T \varepsilon \cdot 8-$

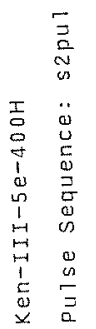




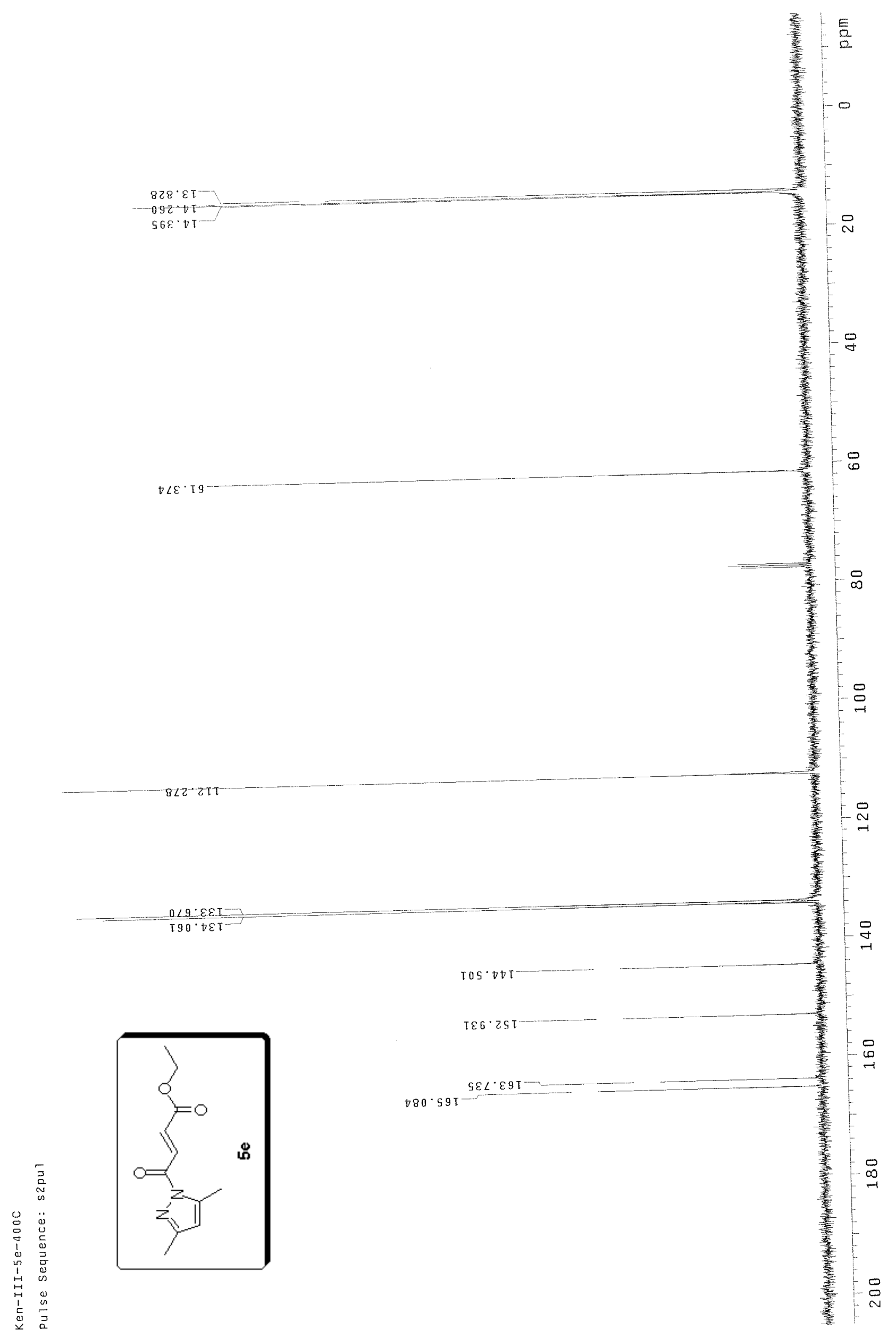





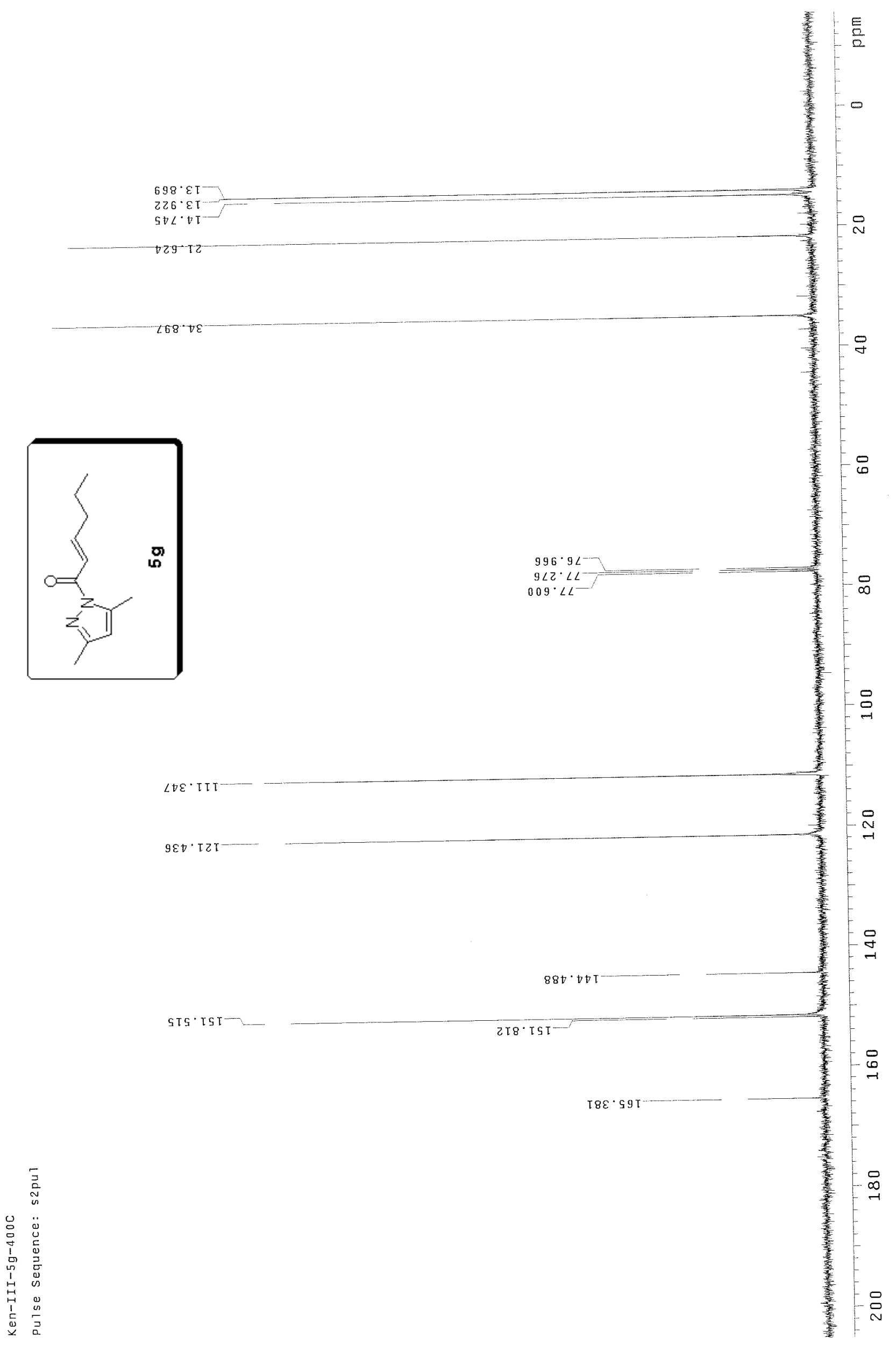



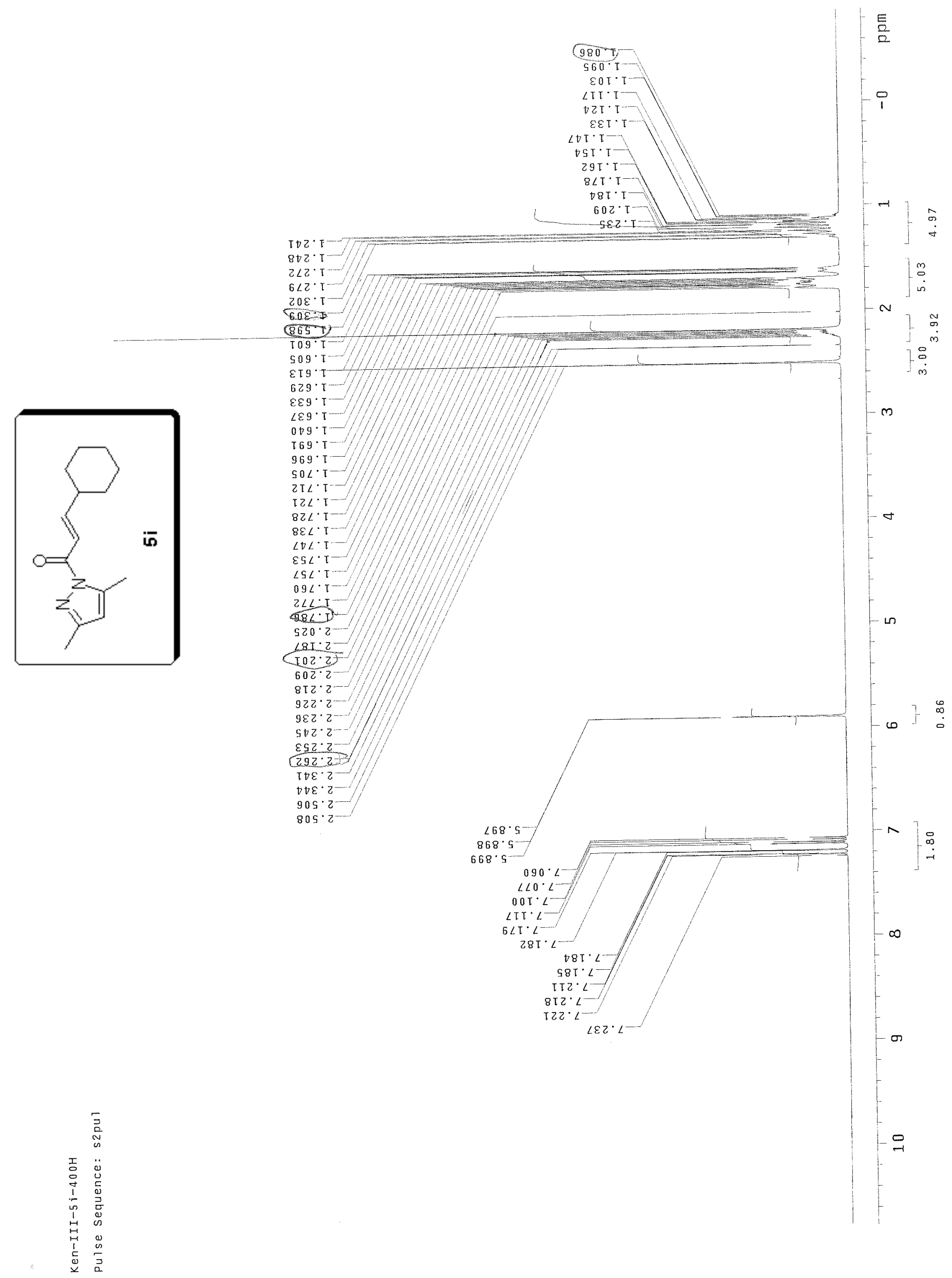


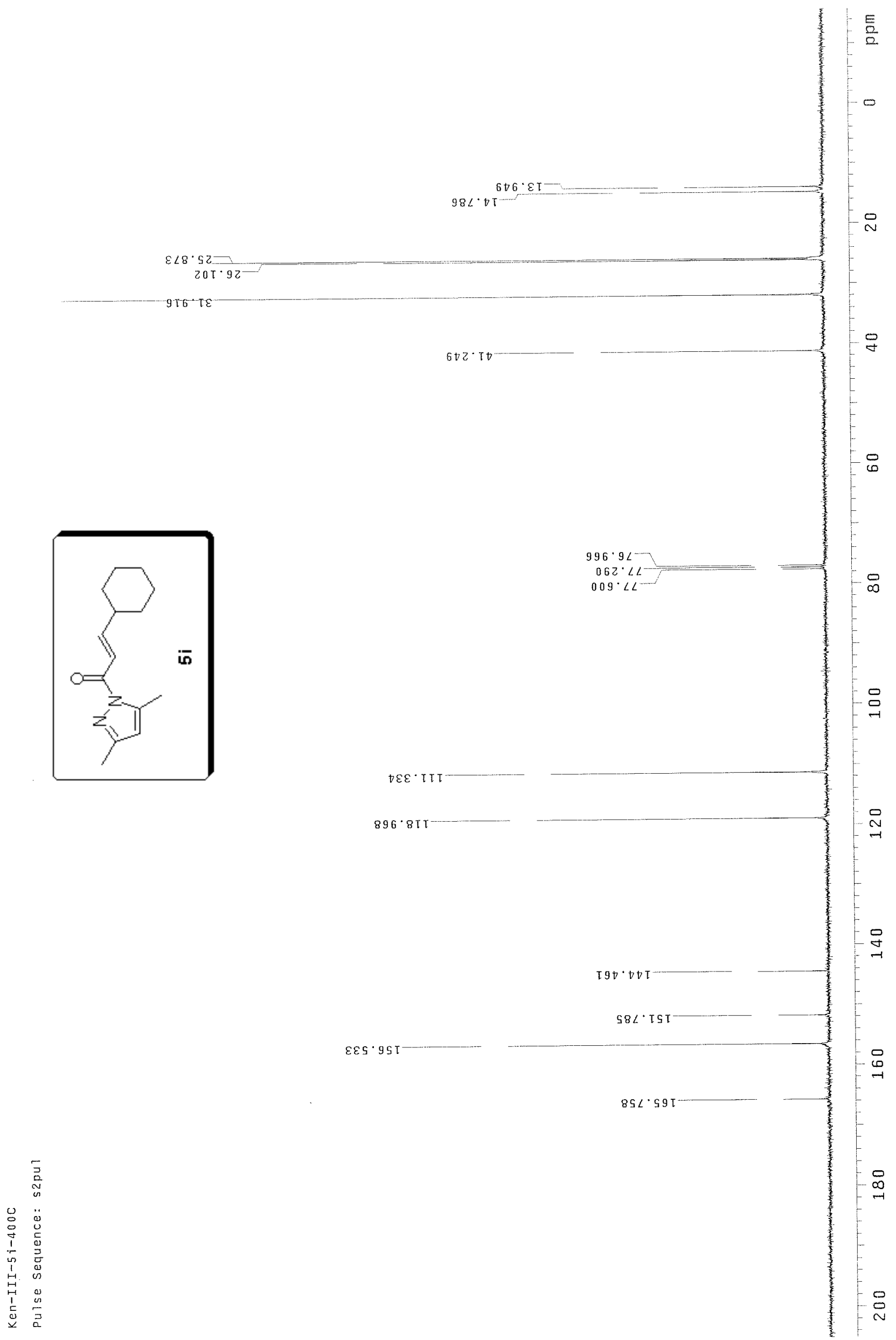


N

655.
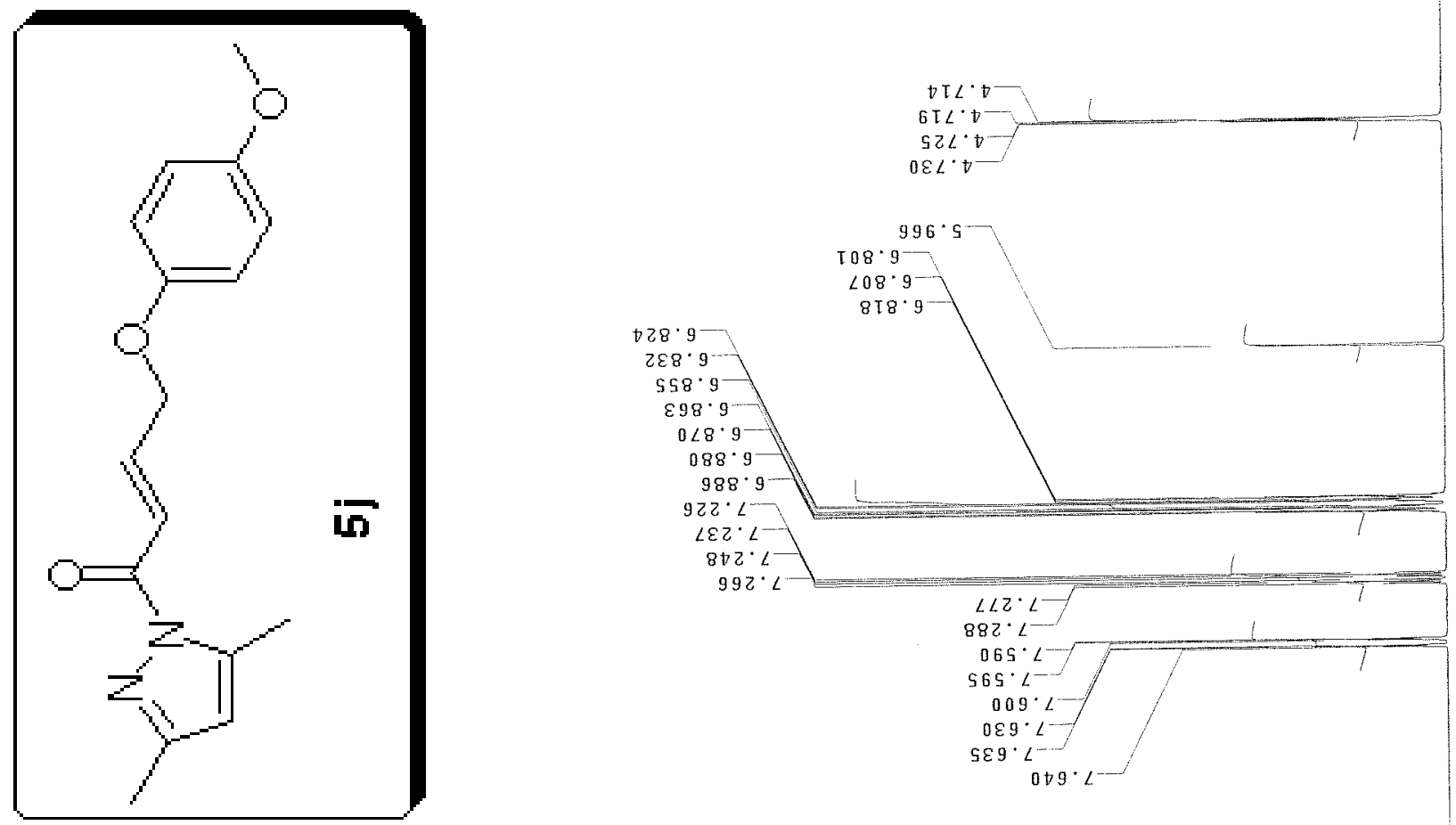

$\sigma$

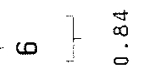

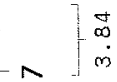

J

0
$-\infty$
0

$\infty$

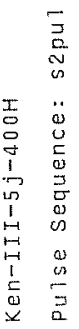

$\stackrel{9}{-1}$ 


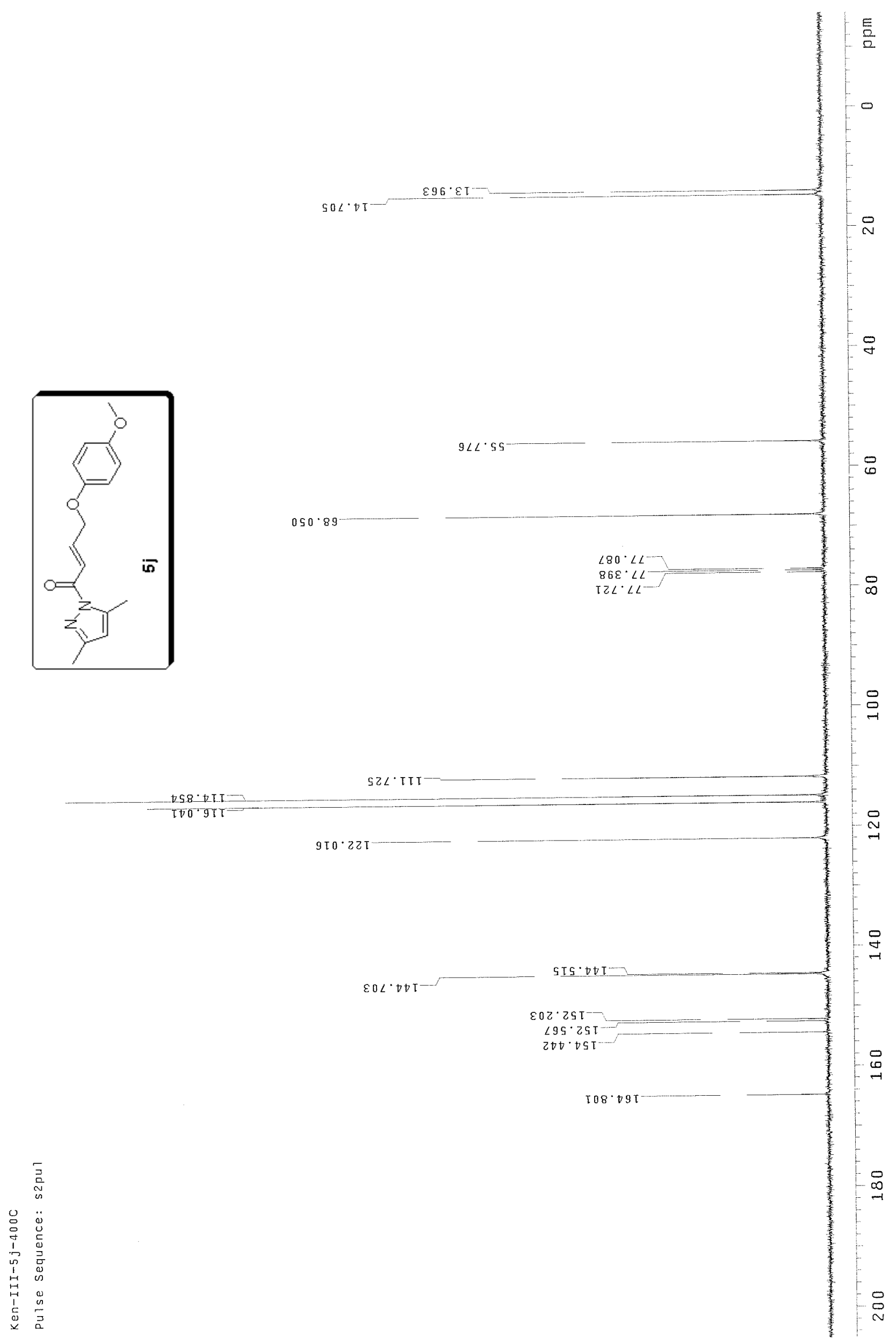



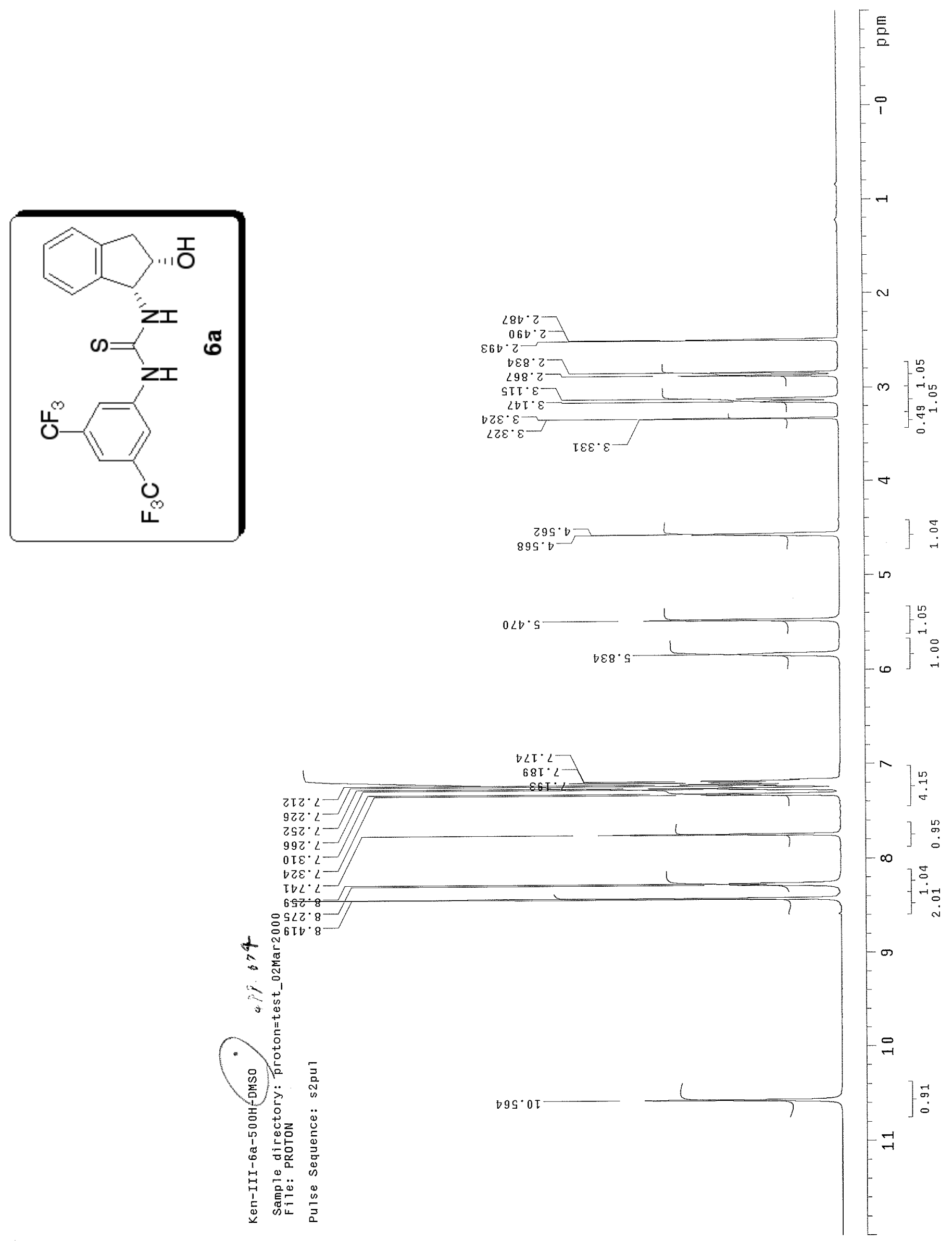


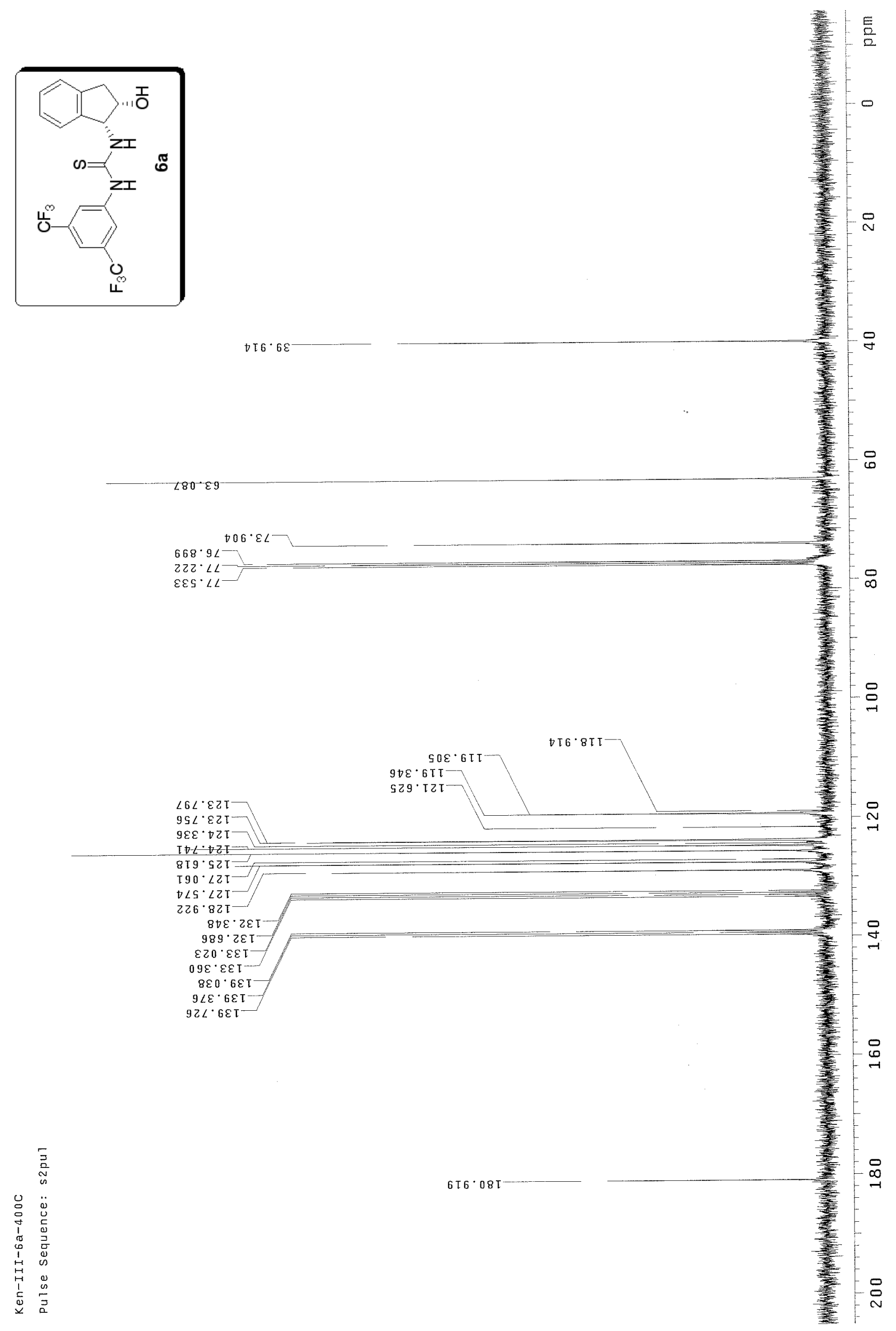



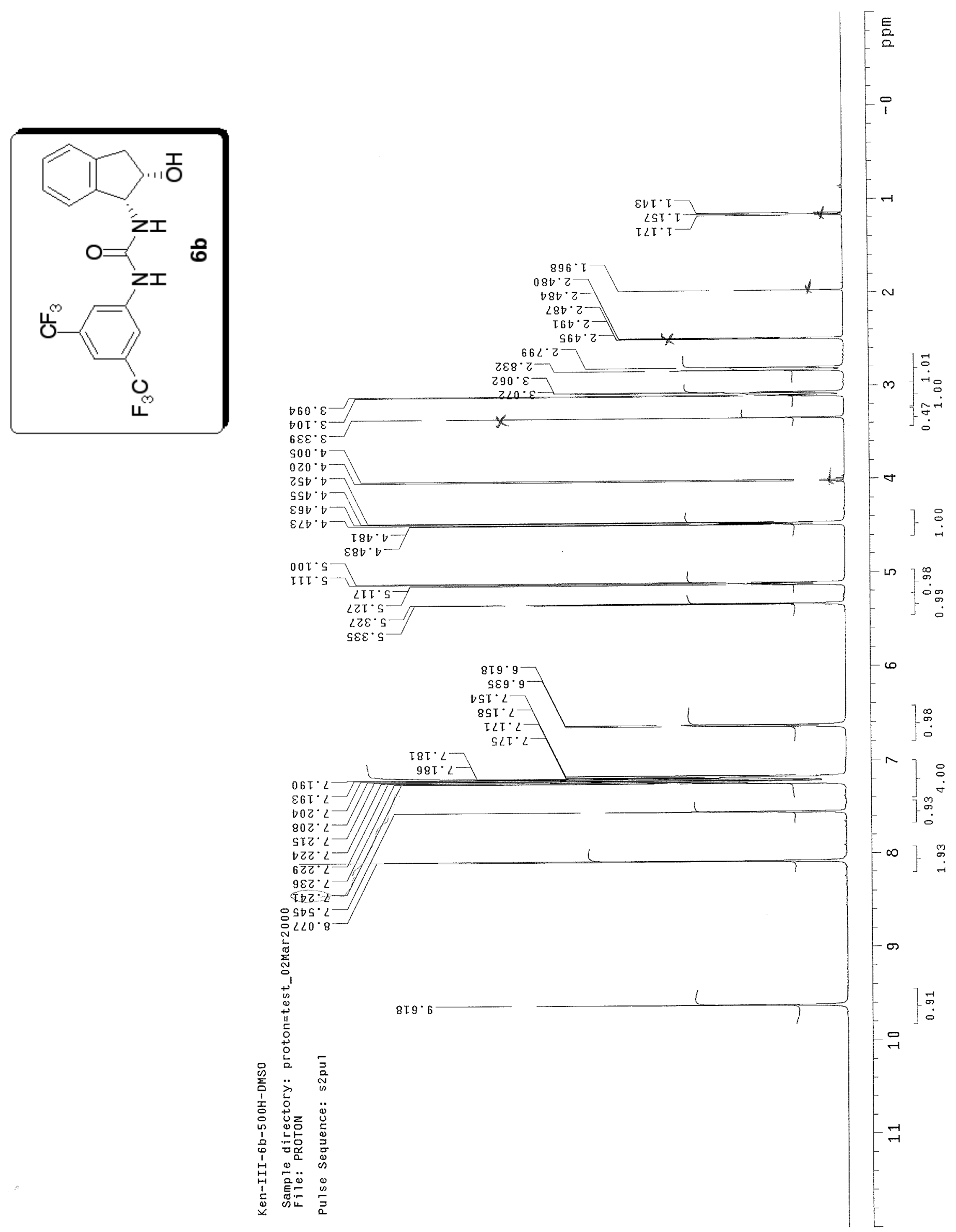

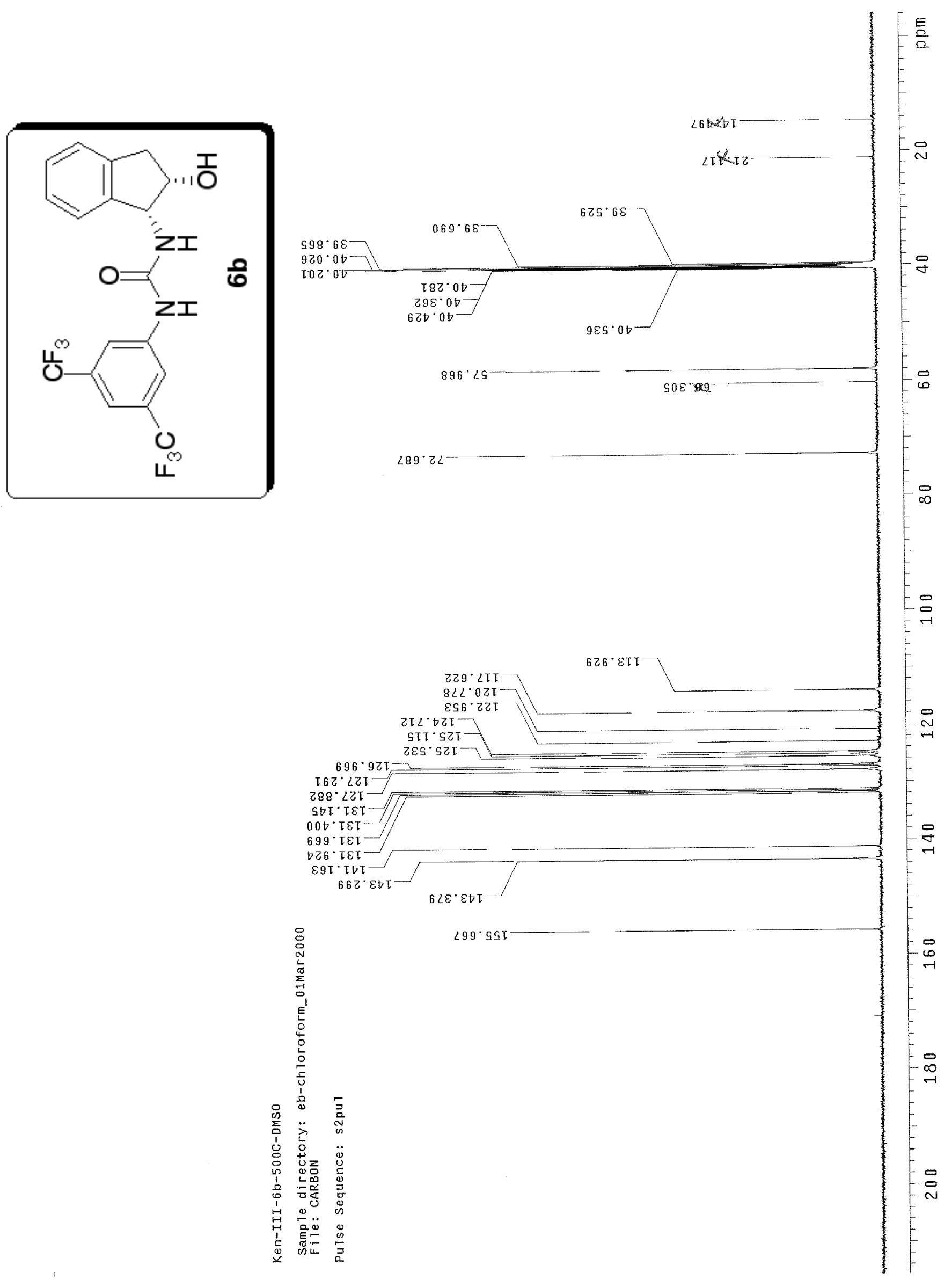

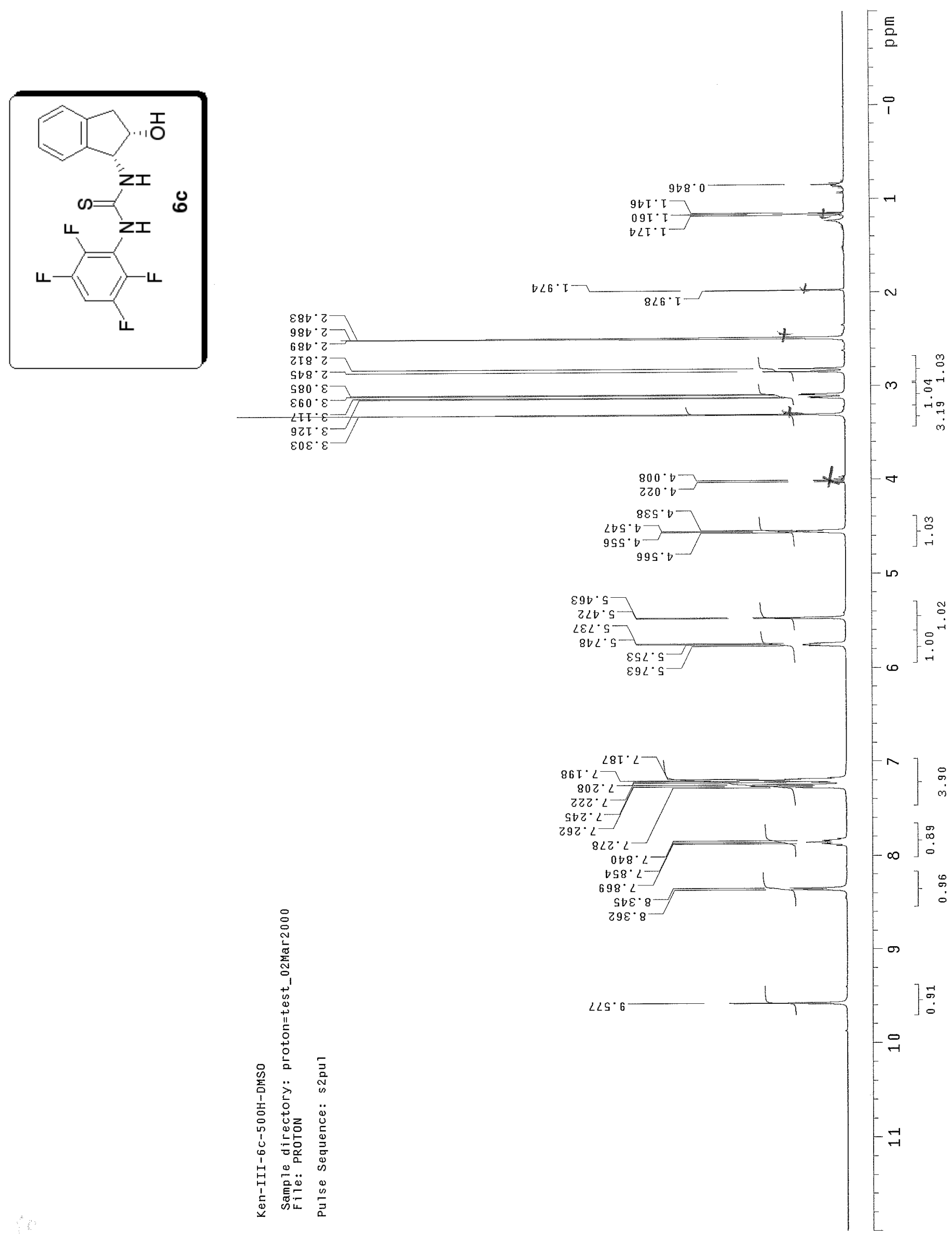

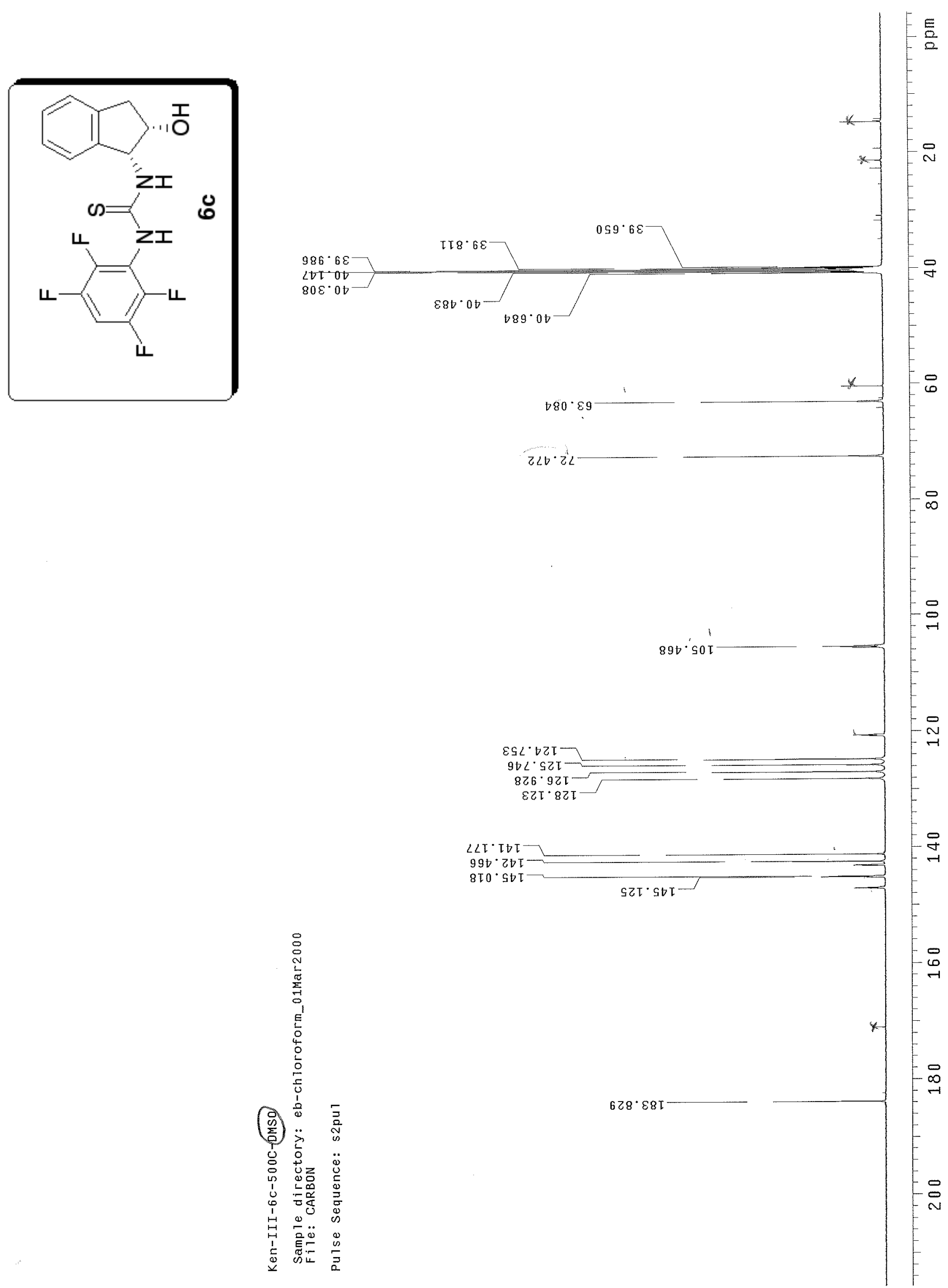


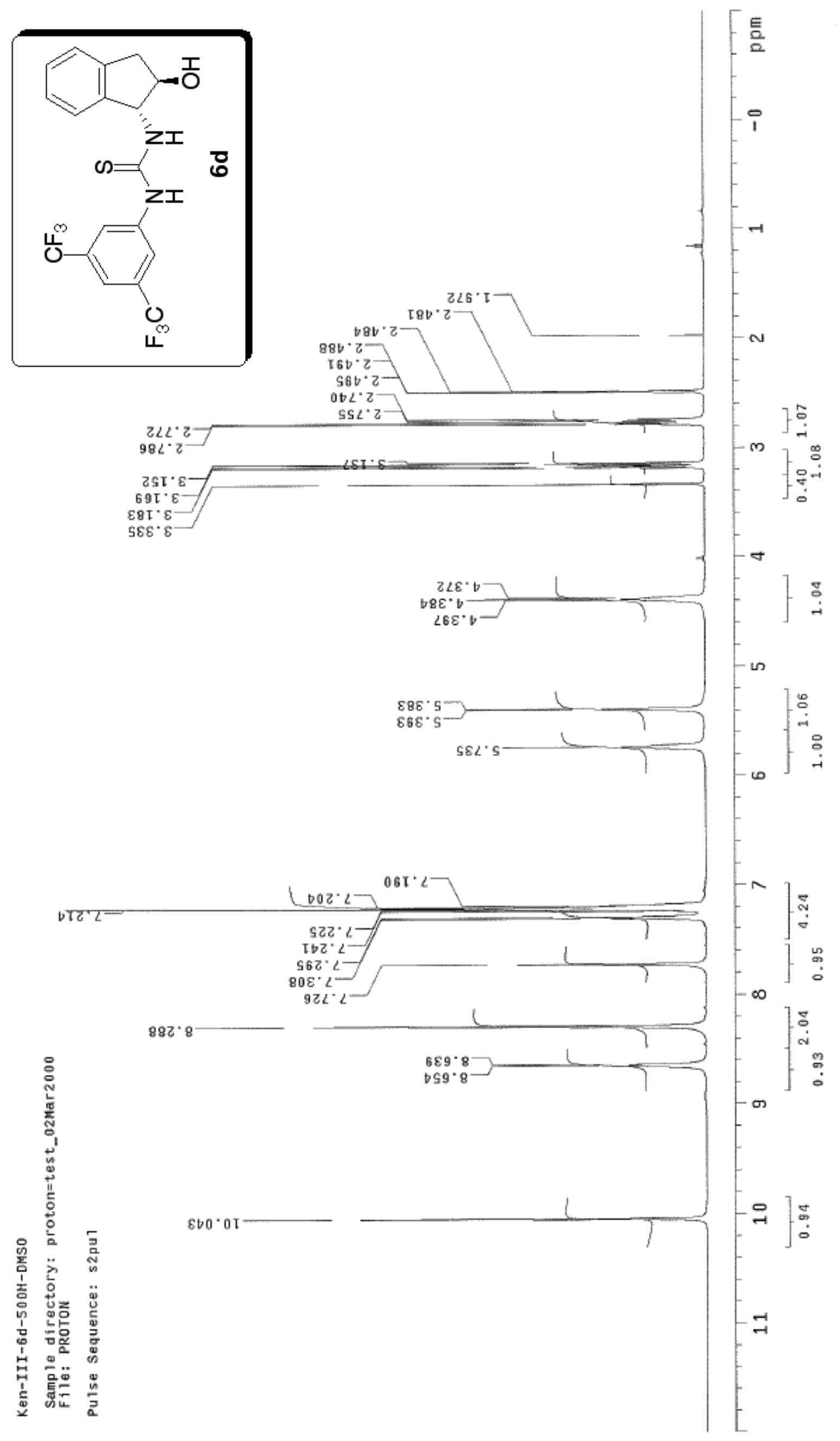



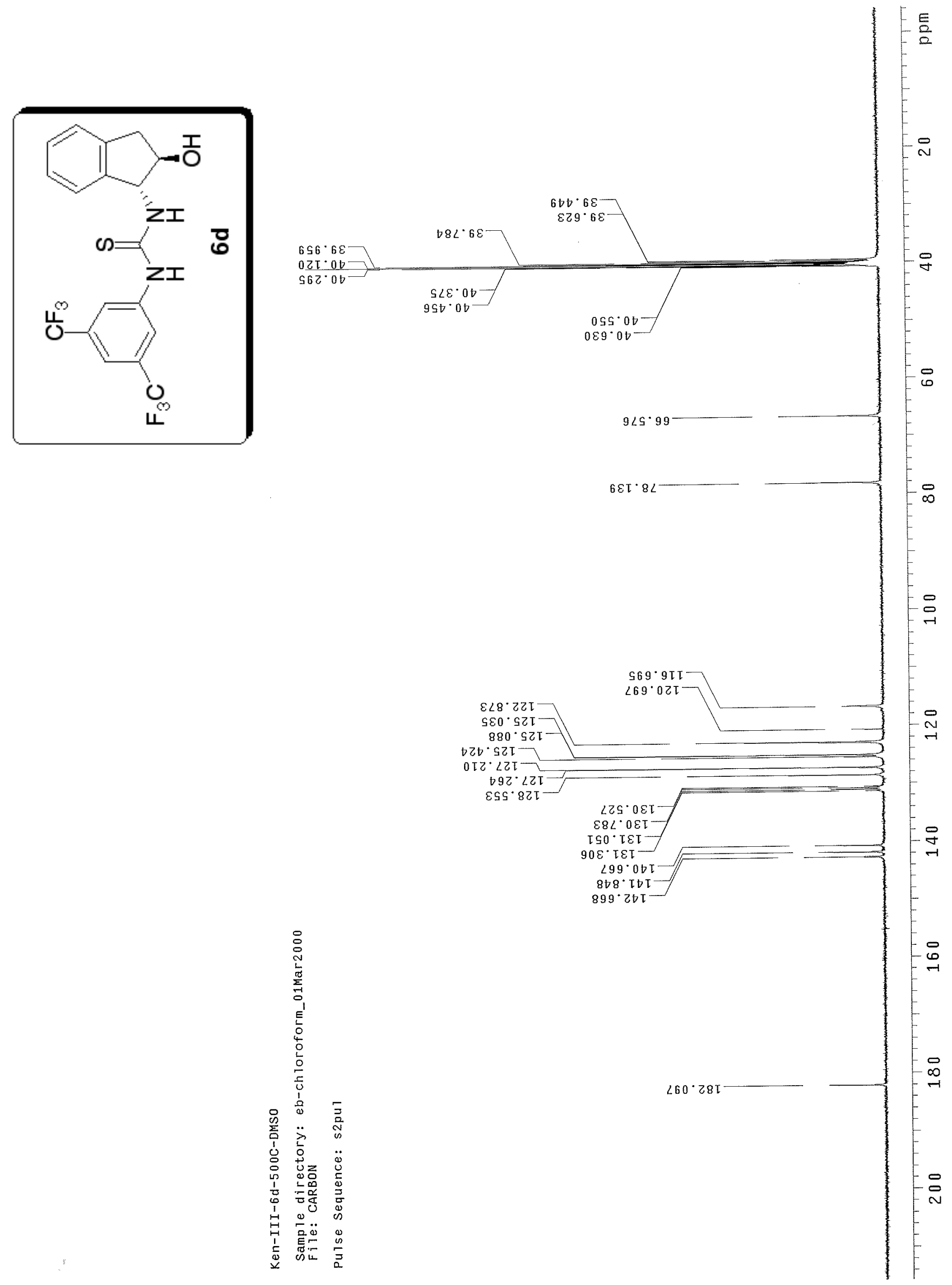

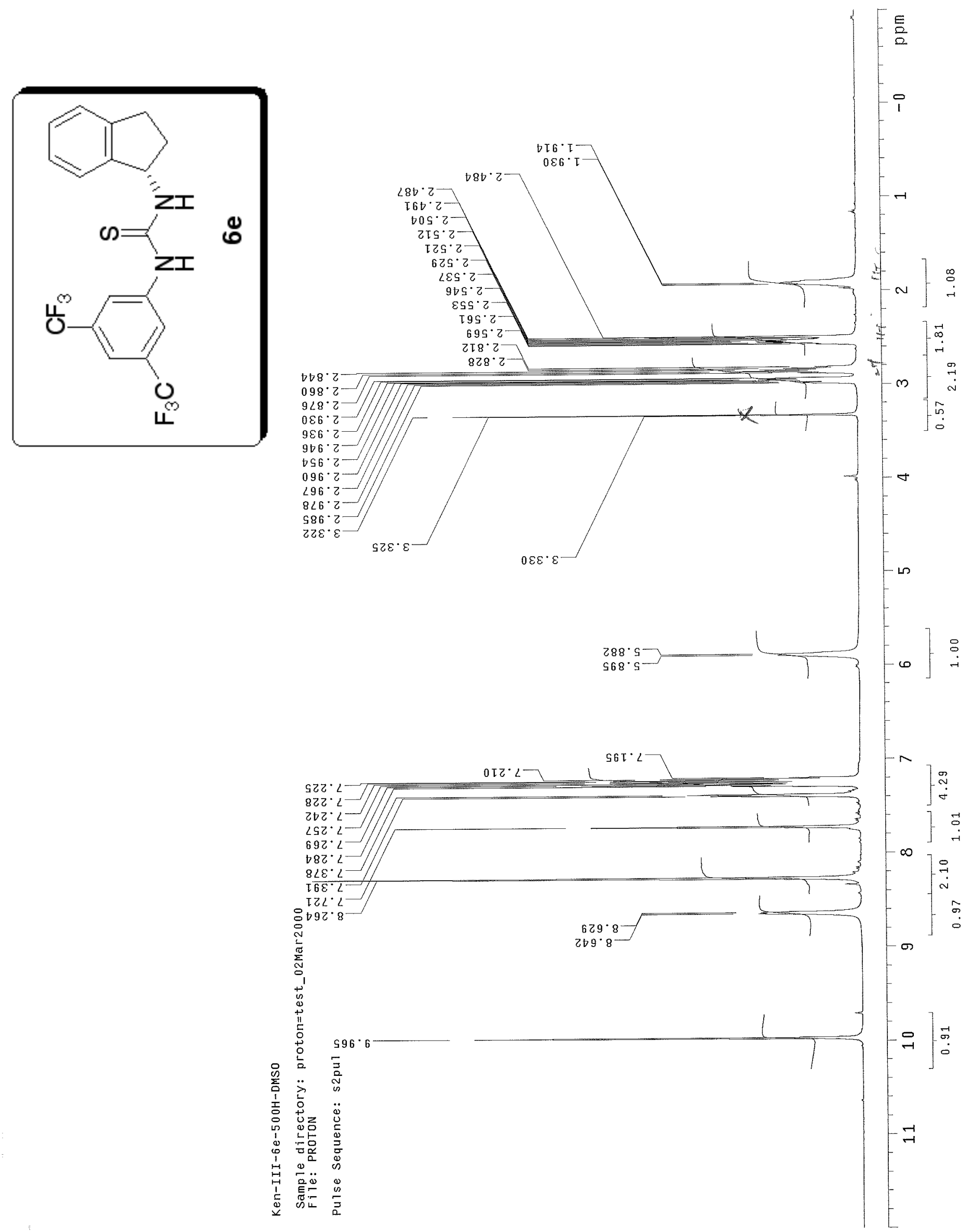

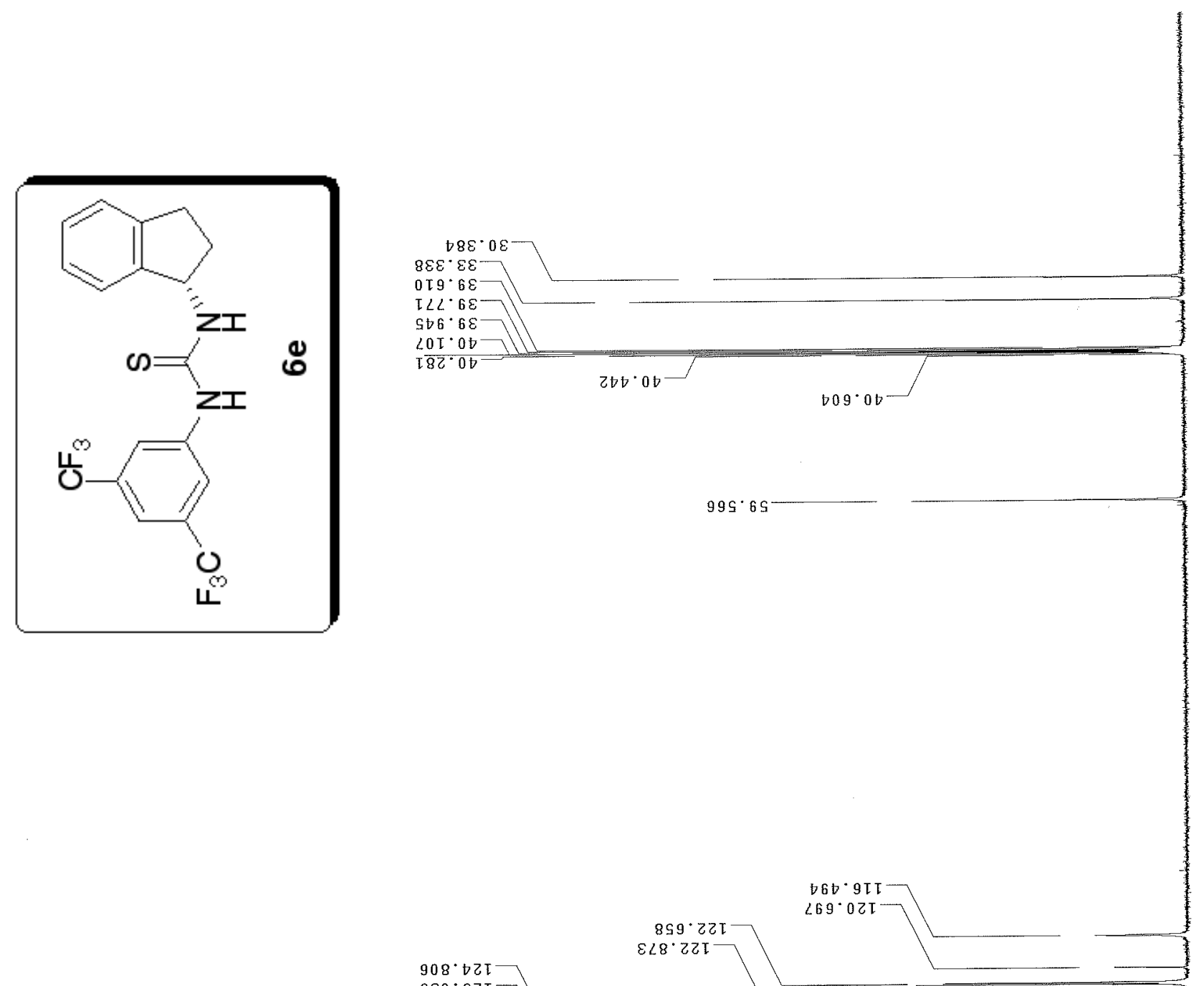

垔

$\stackrel{\sim}{\sim}$
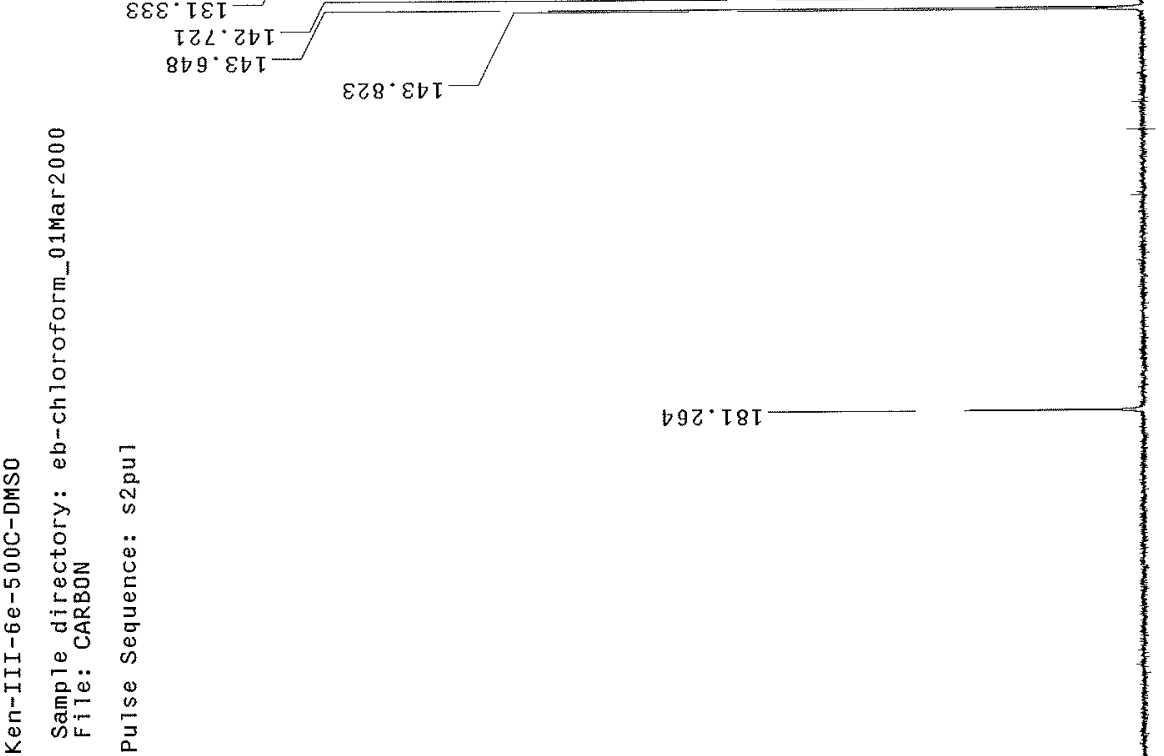

음 

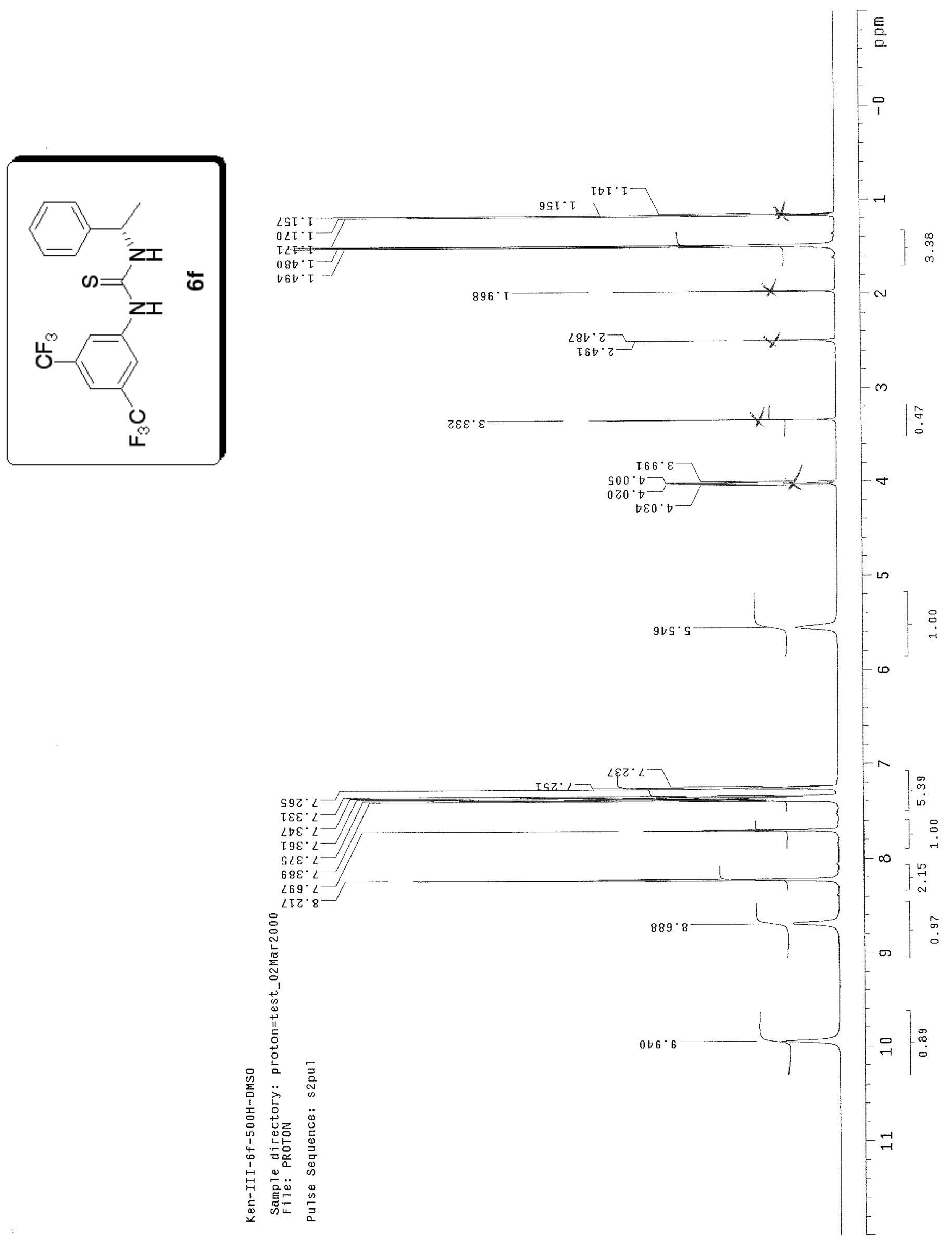

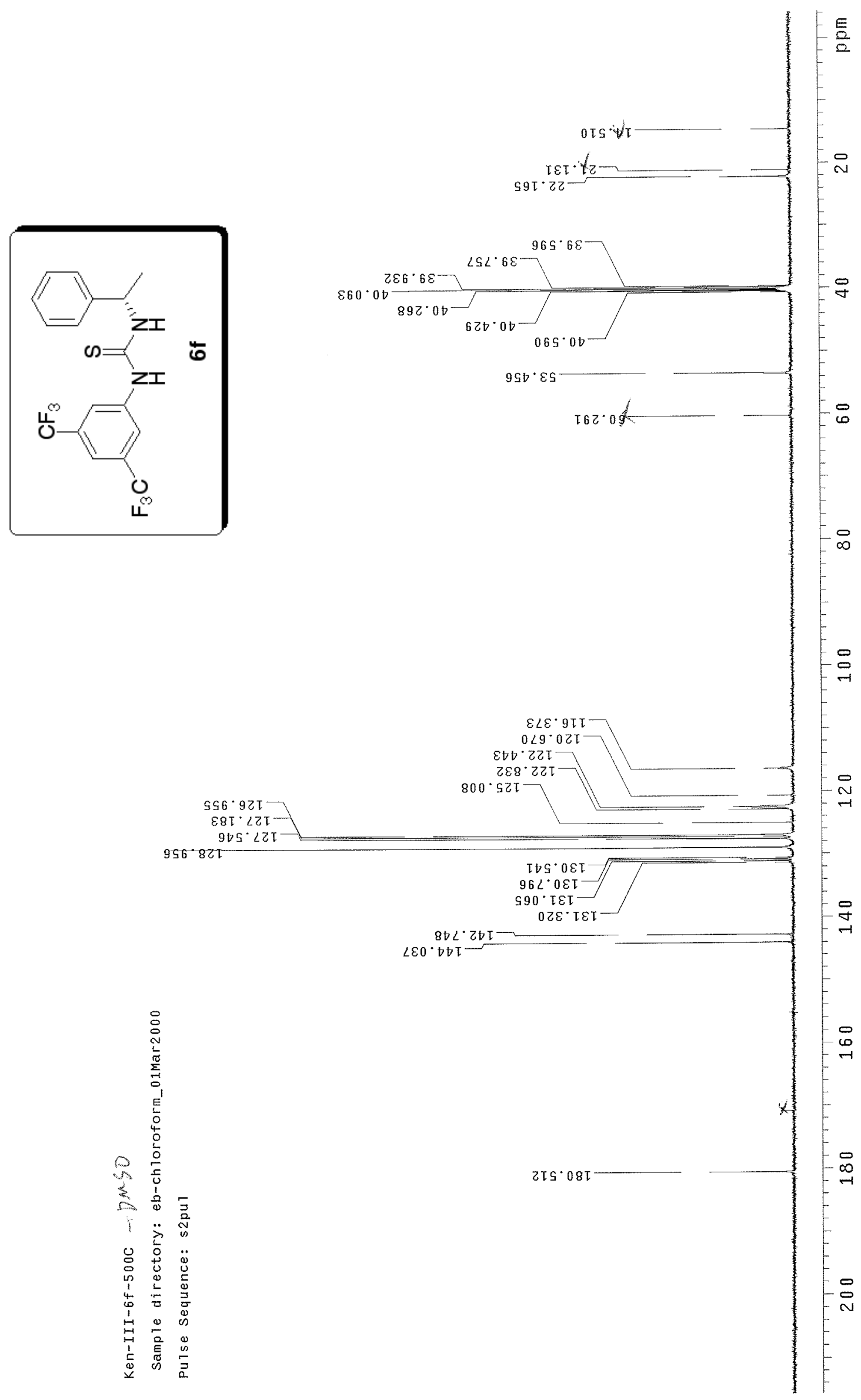

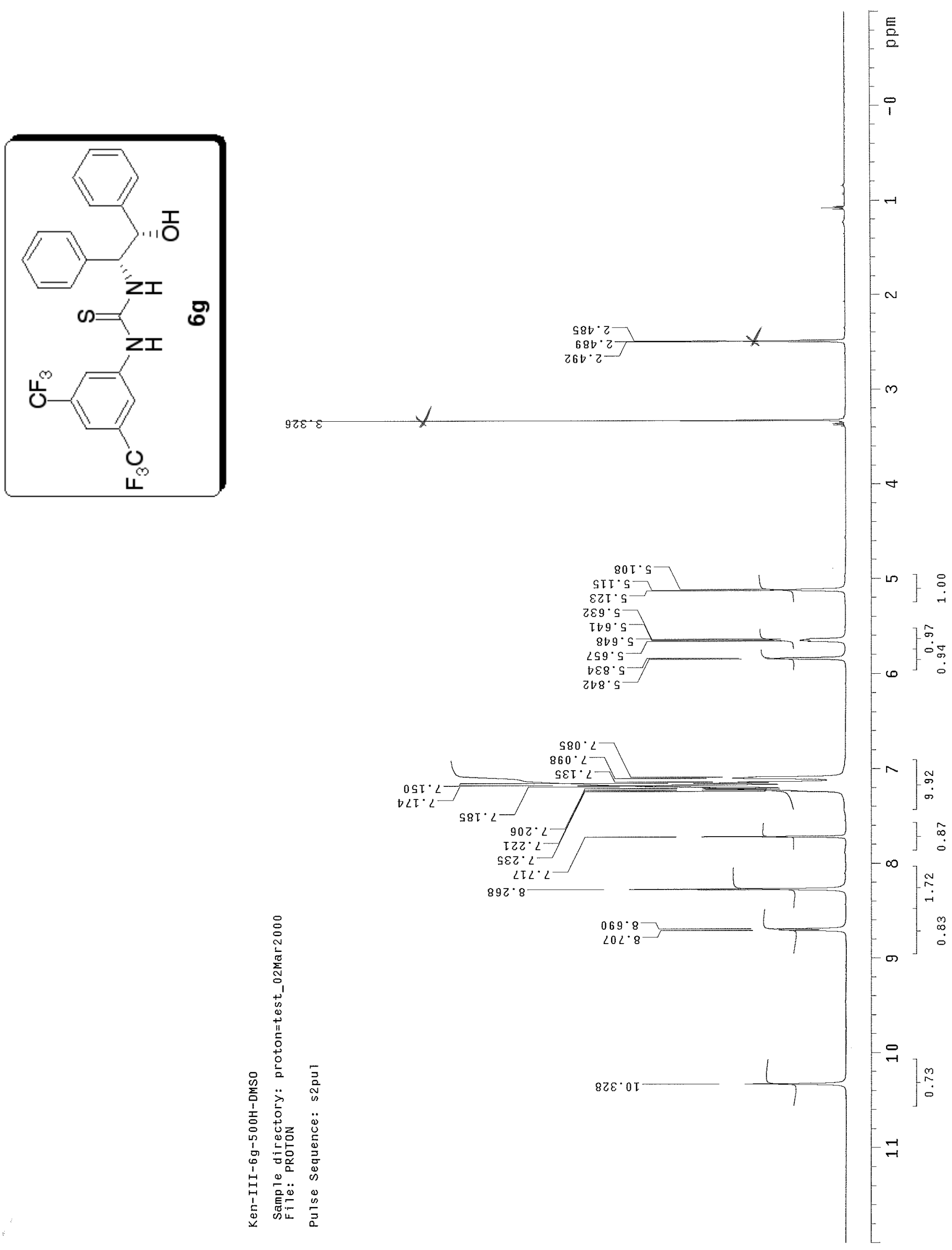

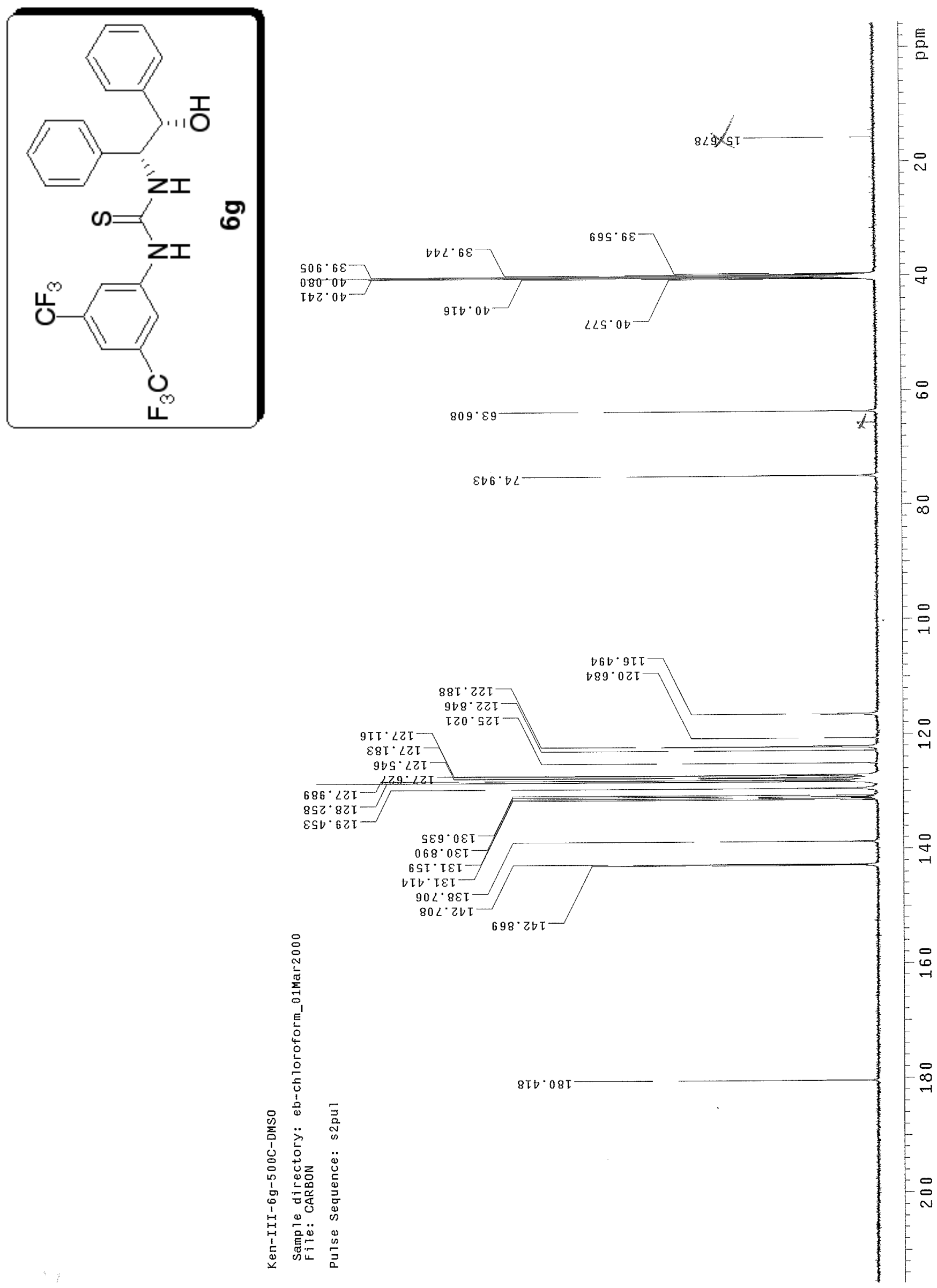

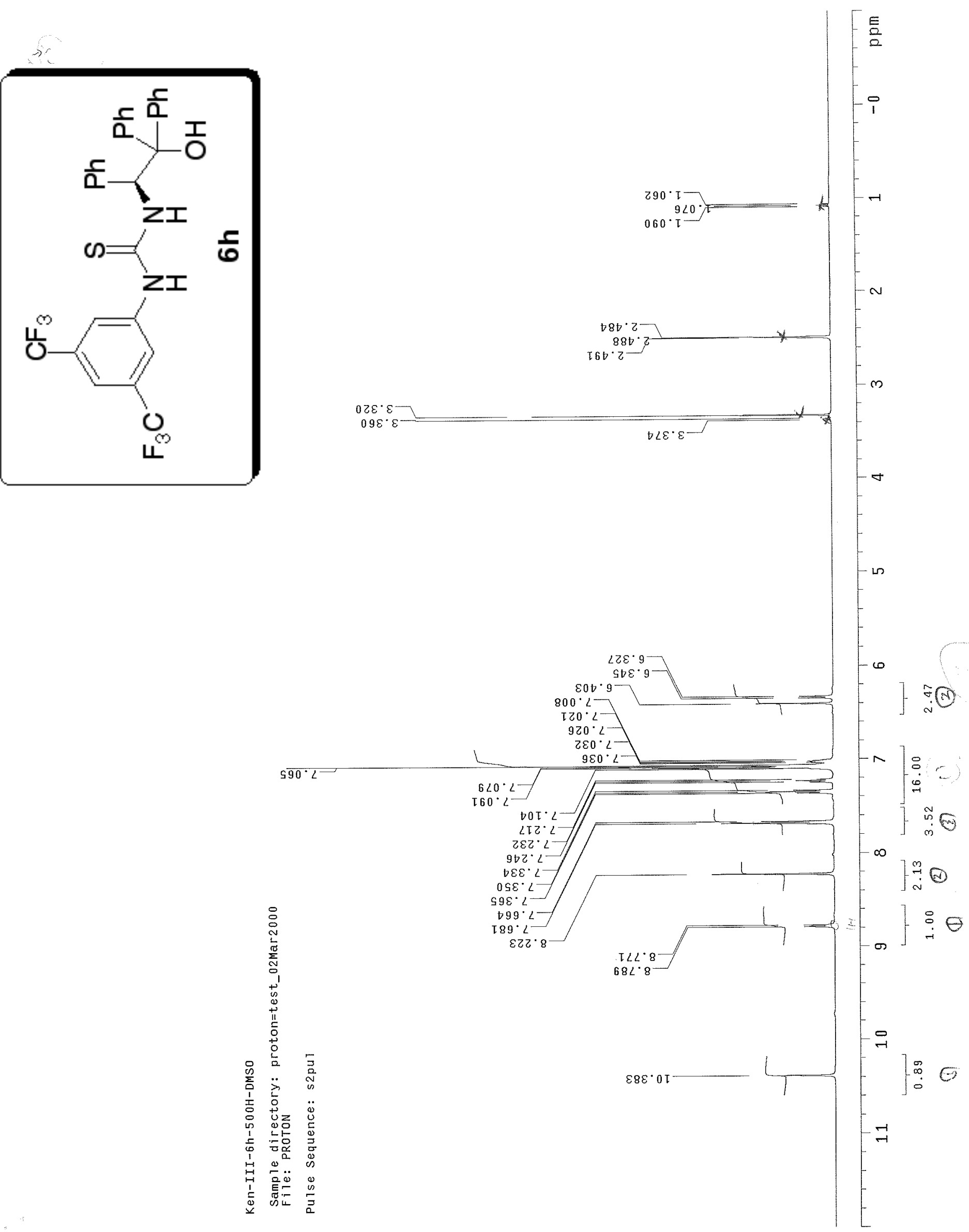

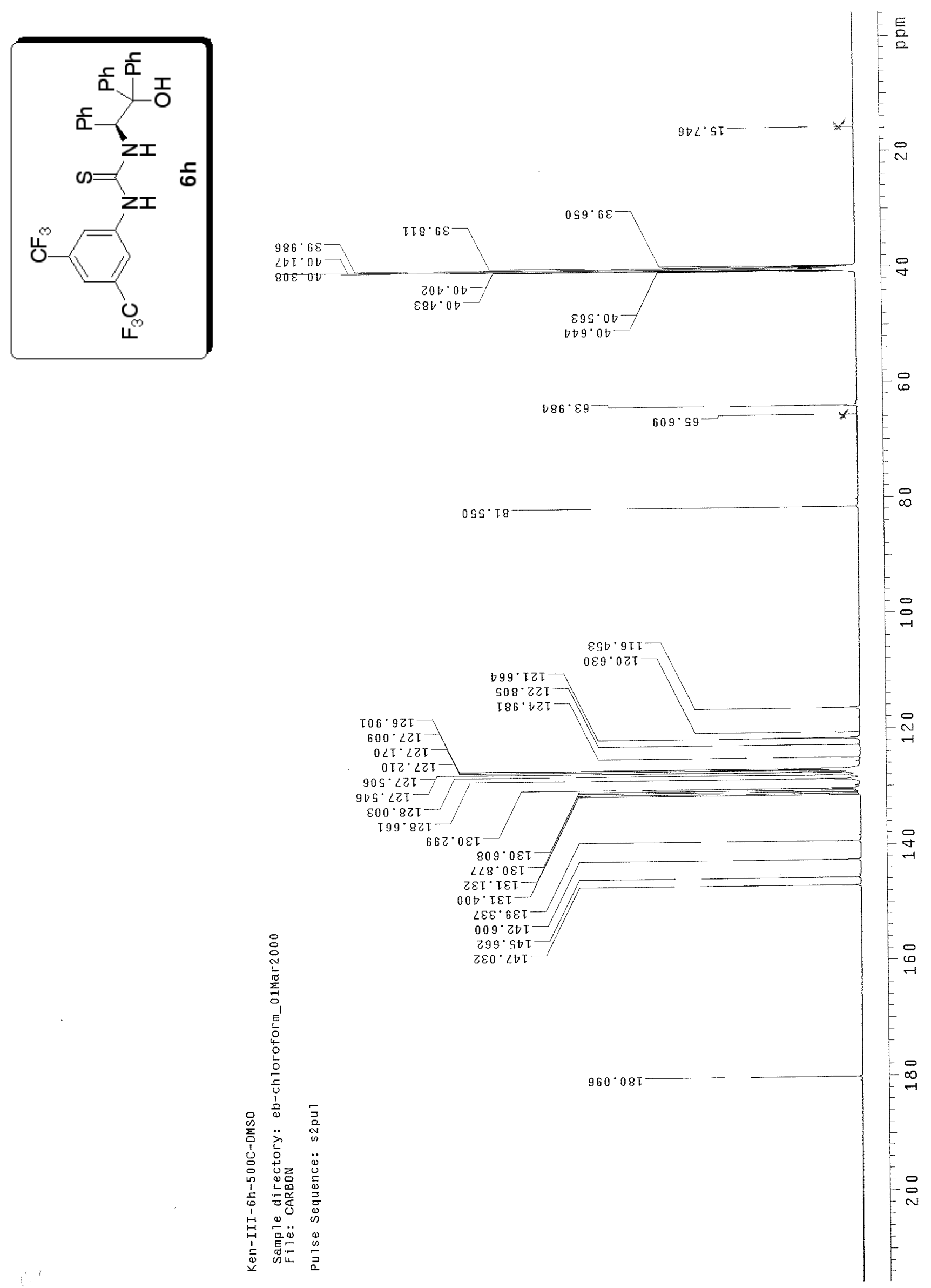

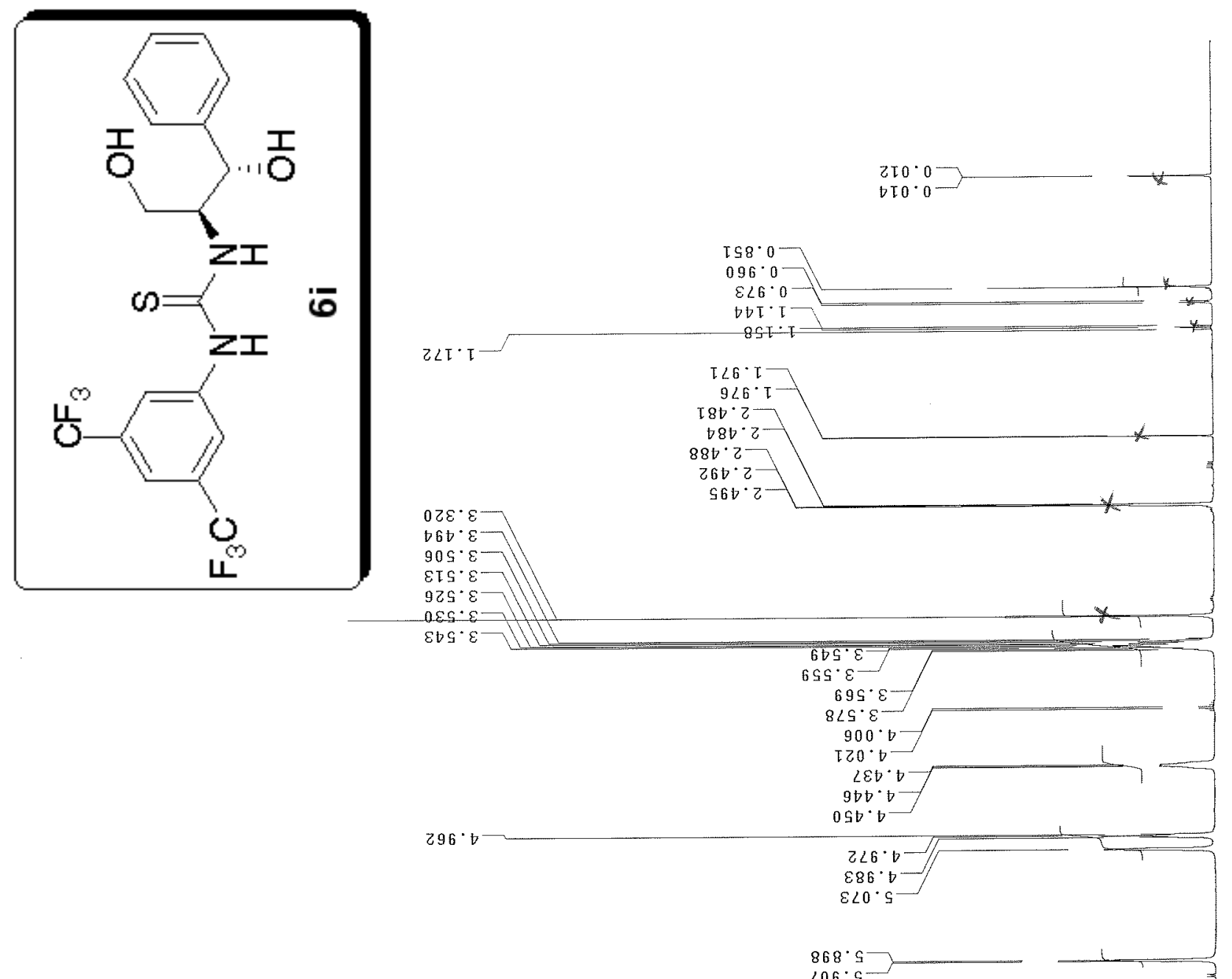

i

5 के

$N$

m

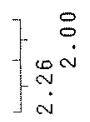

$-\nabla$

]

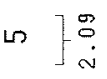

$-\infty] \stackrel{5}{0}$

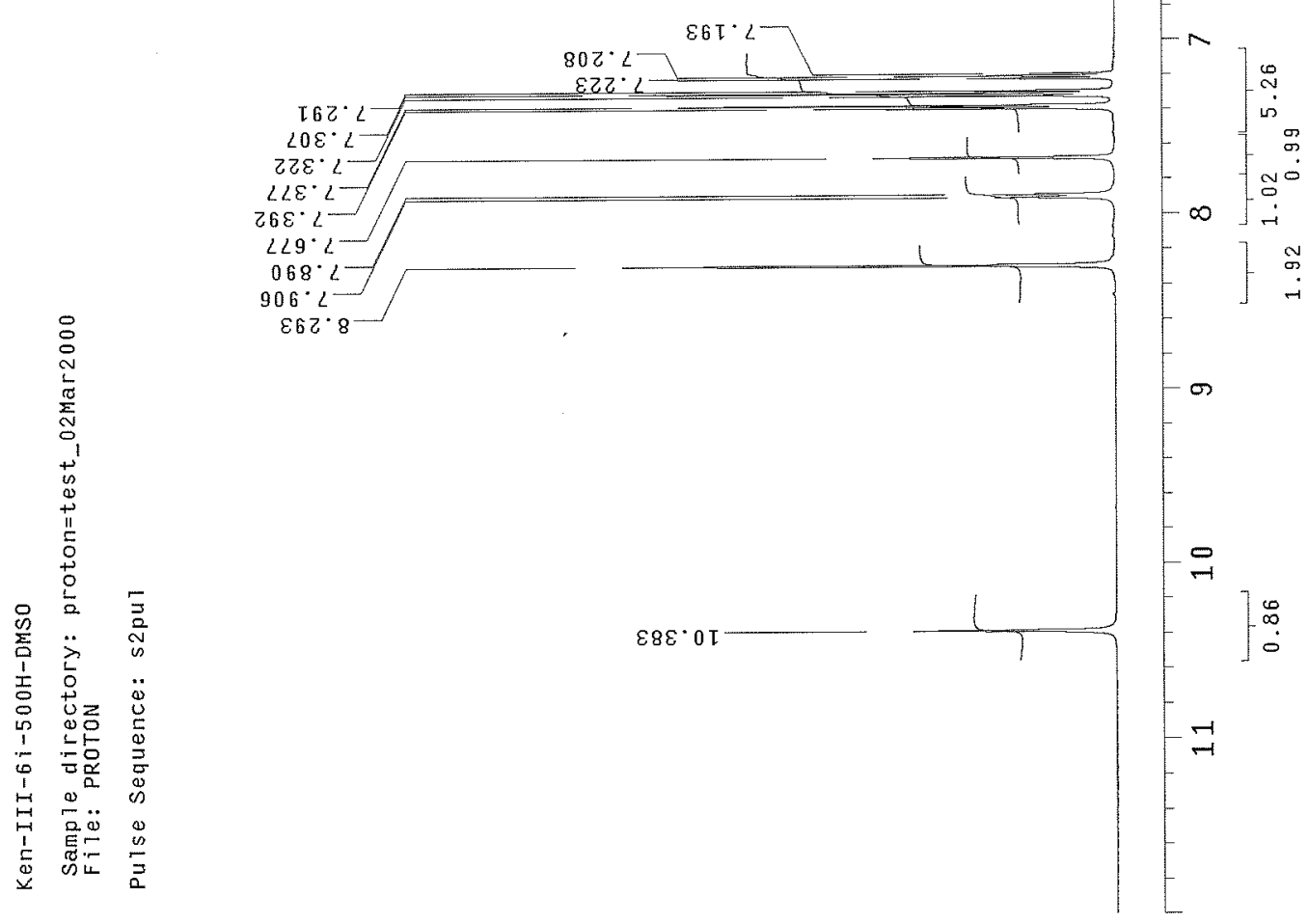



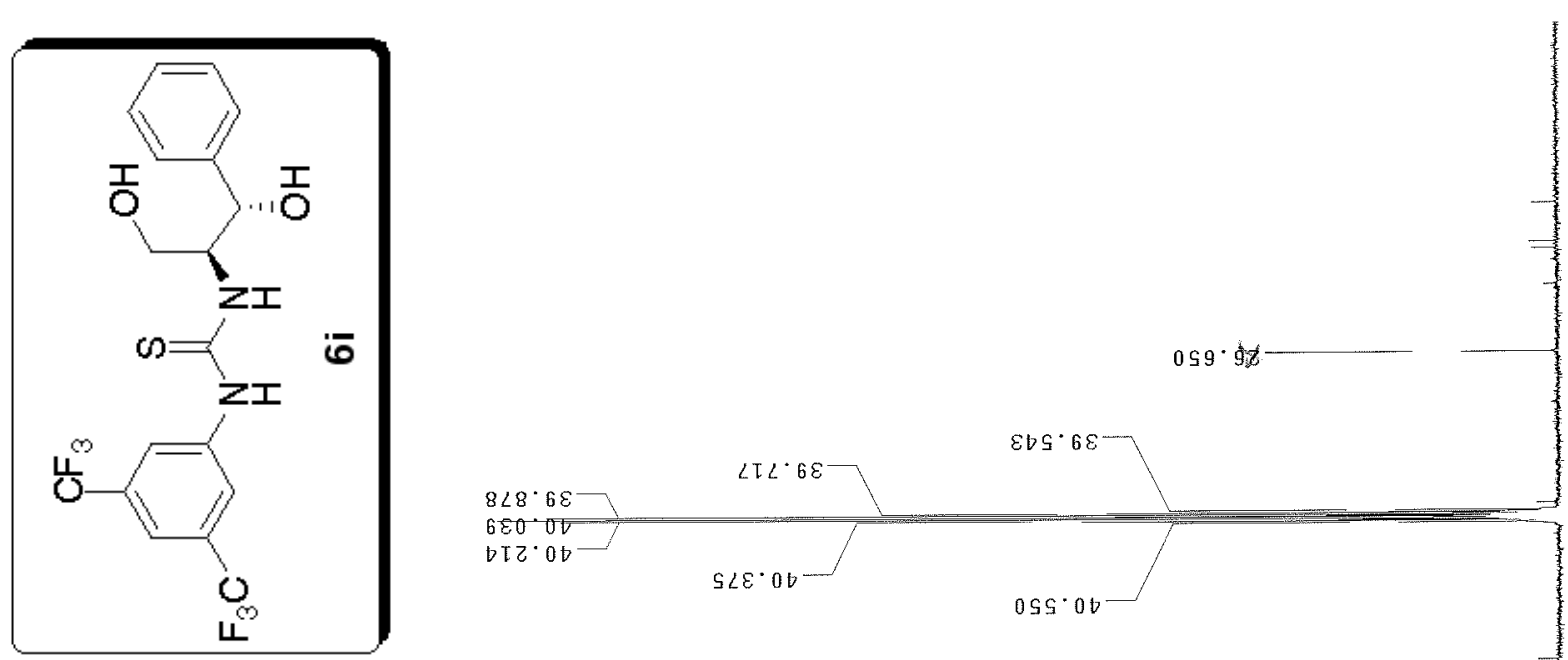
Tseco 9 $I \angle Z^{\circ} T 9$

$\varepsilon 9 D^{\circ} 69$

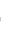

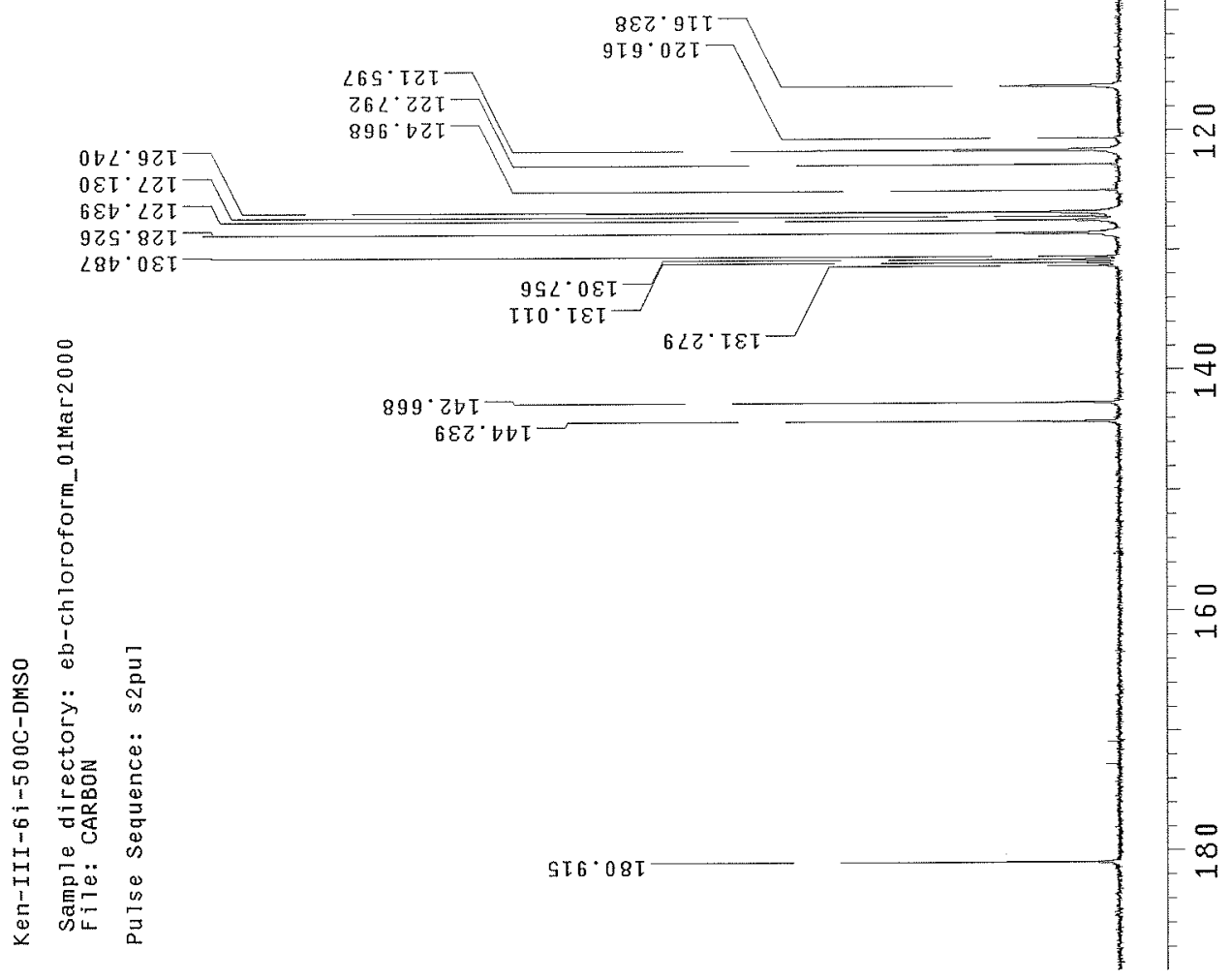




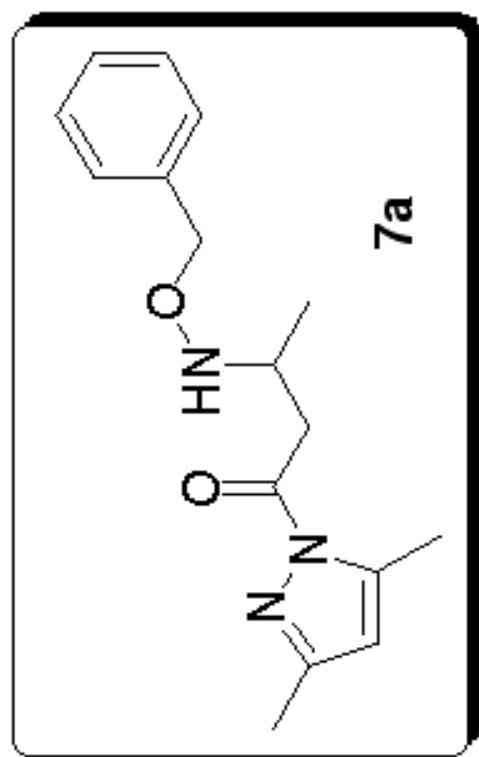

$28 \mathrm{~T} \cdot \mathrm{T}-$

$202 \cdot 1-$

$6 \varepsilon 2$

$56 \mathrm{~T} \cdot 2$

Eoz.

$112 \cdot 2-$

$\angle \angle D^{\circ} \mathrm{Z}$

$080^{\circ}$
$6 \angle 0^{\circ}$

$260^{\circ} \varepsilon$

โ乙

$\varepsilon \varepsilon T \cdot$

$\tau \angle \varepsilon^{\prime} \varepsilon$

$56 \varepsilon \cdot \varepsilon$

จT० 8

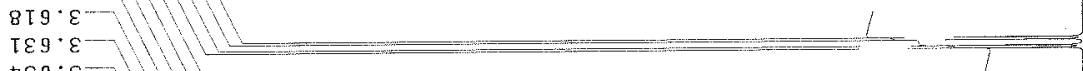

$\nabla \varepsilon 9 \cdot \varepsilon$

$\angle 09 \cdot \varepsilon^{-}$

Tร9.

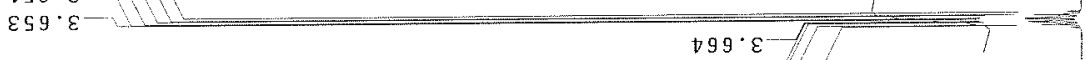

i

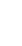

$-1$

9
0
0

$N$

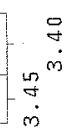

$m$

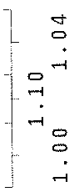

$199 \cdot 6$

$\stackrel{\circ}{\sim}$

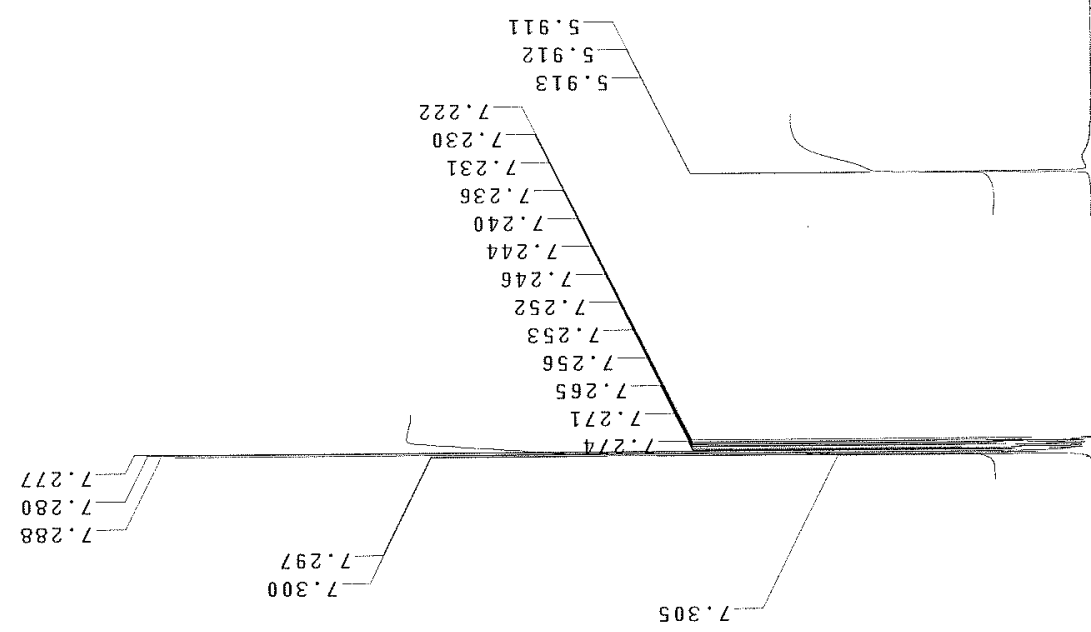

เ

- $\left[\begin{array}{l}0 \\ 0 \\ 0 \\ -1\end{array}\right.$

$N$ $\int \frac{8}{8}$ 


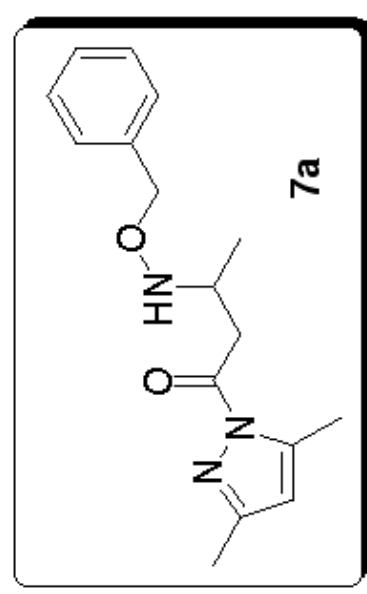

$869 \cdot 68$

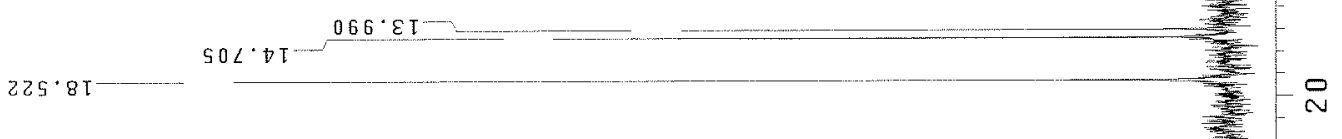

I $6 \angle{ }^{\circ} 9 L^{-}$

$926 \cdot 9 L$

$602 \cdot \angle L$

$\angle 0 \varepsilon^{\circ} \operatorname{TT}$

$088^{\circ} \angle Z T$ $8 T 5 \cdot 8 Z T$

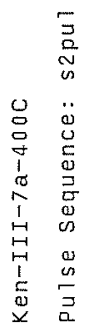

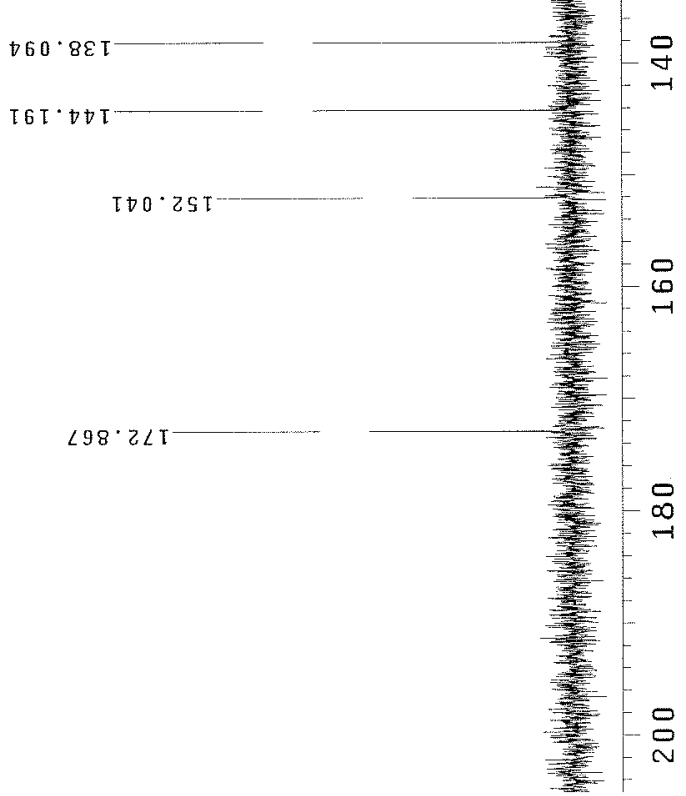




\section{Sample Information}

$\begin{array}{llll}\text { SampleName } & \text { Kll5280J10\%1m } & \text { Sample Type } & \text { Unknown } \\ \text { Vial } & 1 & \text { Date Acquired } & \text { 8/30/2004 5:59:26 PM } \\ \text { Injection } & 2 & \text { Acq Method Set } & \text { 10\%iPrOH 1mpm } \\ \text { Injection Volume } & 10.00 \mathrm{ul} & \text { Processing Method Kll5280J10\%1m } \\ \text { Channel } & \text { 2487Channel 1 } & \text { Date Processed } & \text { 8/30/2004 7:35:08 PM } \\ \text { Run Time } & \text { 240.0 Minutes } & \end{array}$

Auto-Scaled Chromatogram

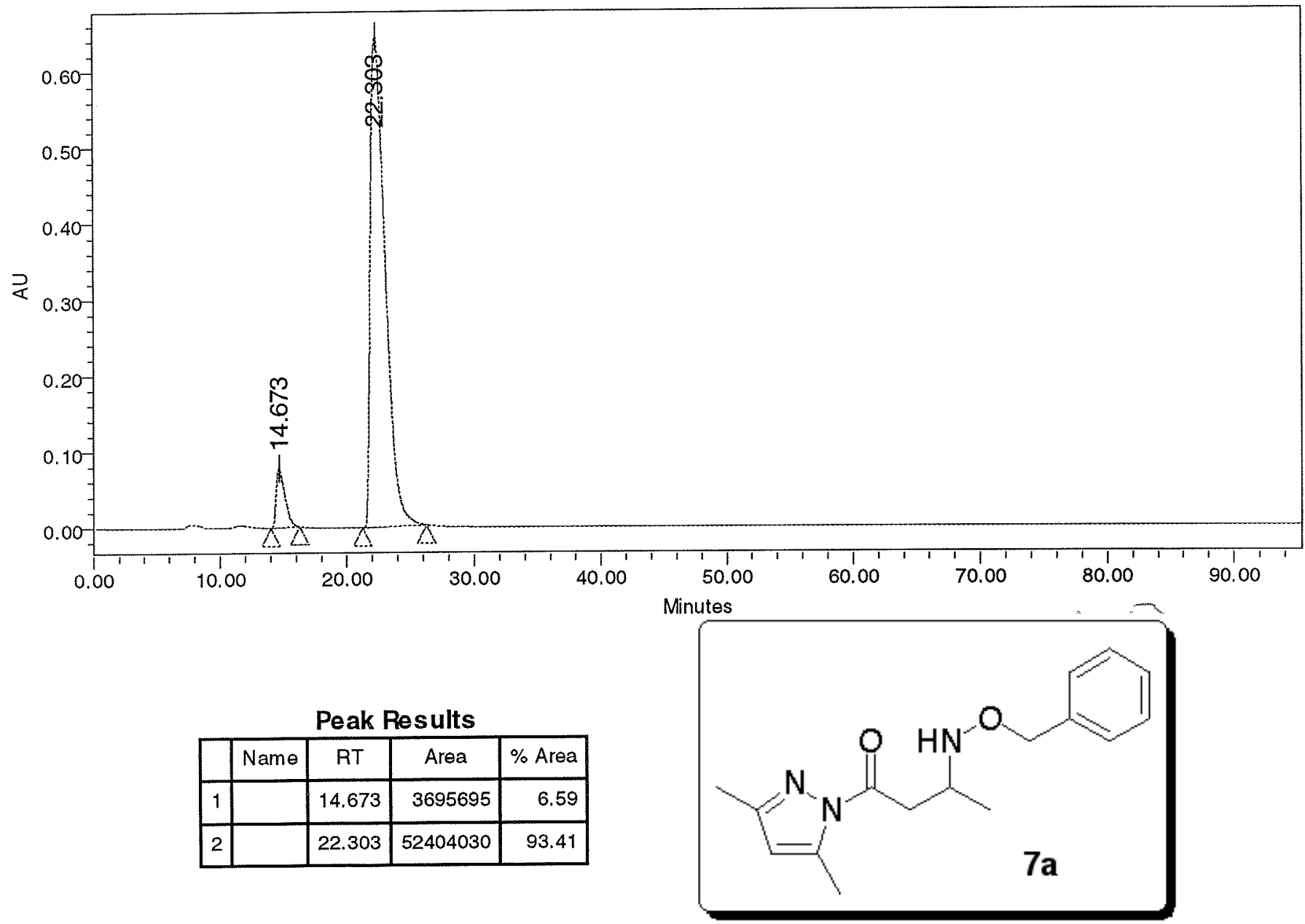



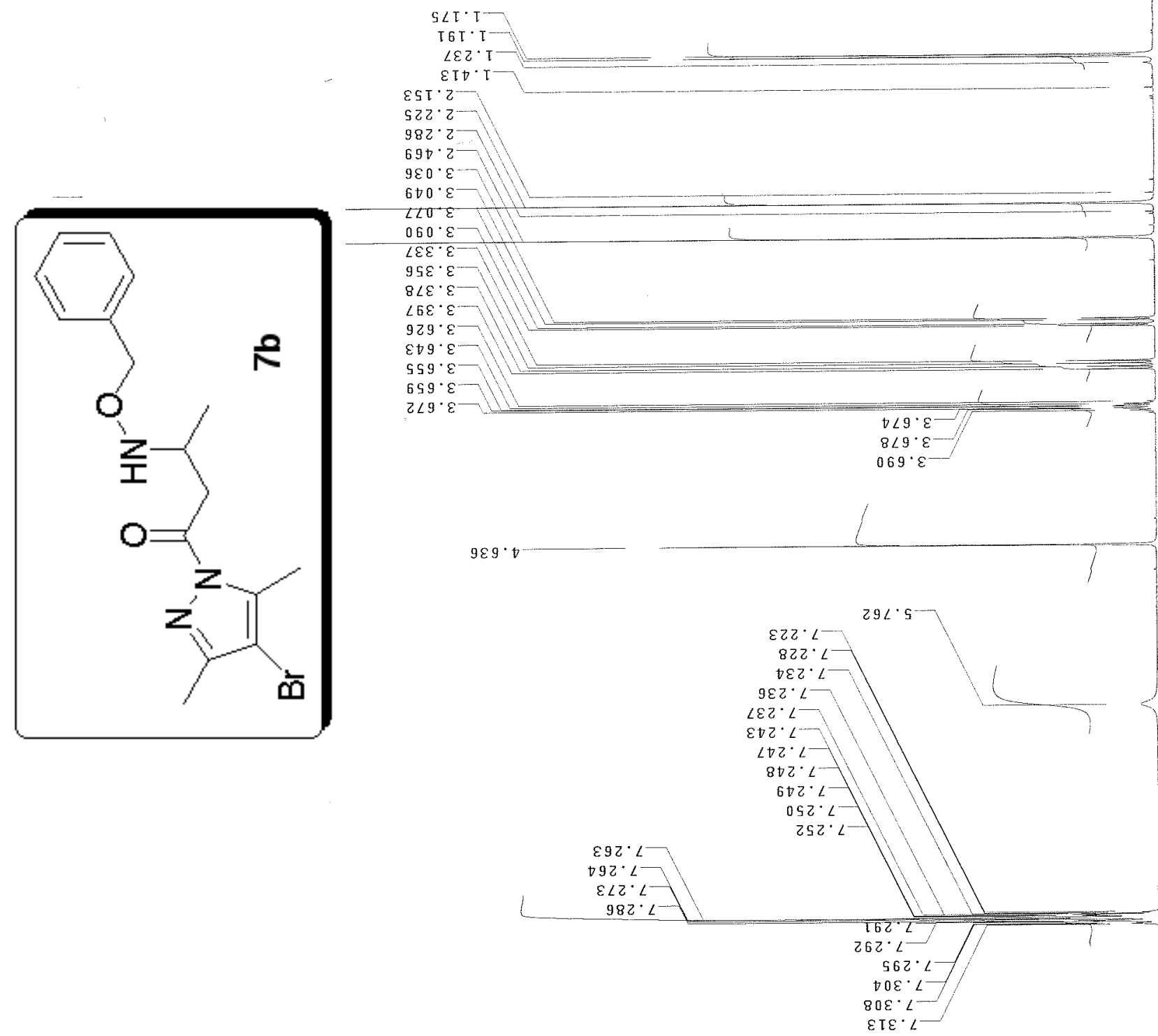

7

$N$

我

$-m$

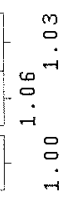

$-\sigma$

울

- n

$\infty$
$\infty$
0
0

$\infty$

N

的

$\infty$

$\pi$ 

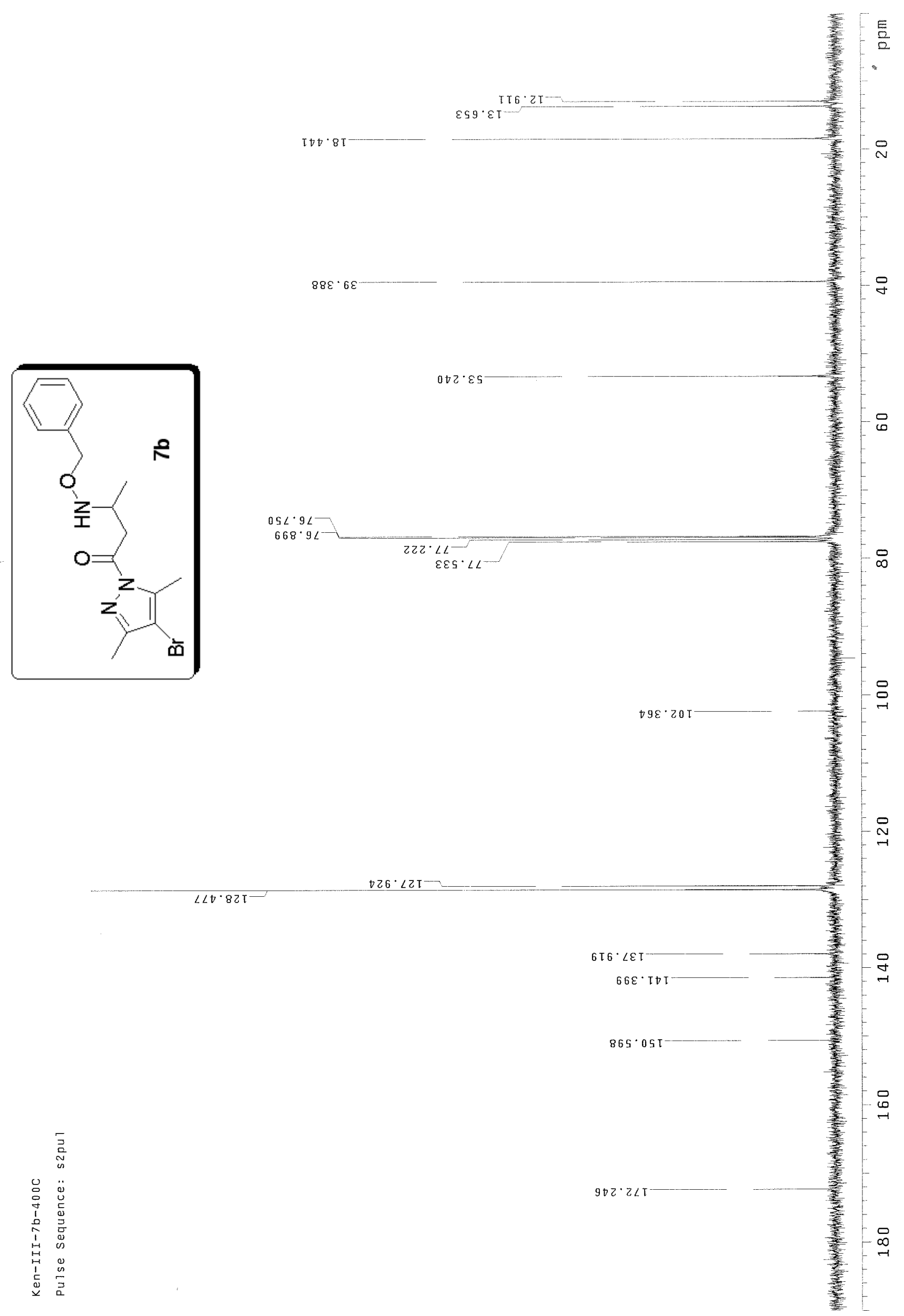


\section{Sample Information}

$\begin{array}{llll}\text { SampleName } & \text { Kll539ODH10\%1m } & \text { Sample Type } & \text { Unknown } \\ \text { Vial } & 1 & \text { Date Acquired } & \text { 9/2/2004 10:33:40 AM } \\ \text { Injection } & 1 & \text { Acq Method Set } & \text { 10\%iPrOH 05mpmH3 } \\ \text { Injection Volume } & 10.00 \mathrm{ul} & \text { Processing Method Kll539ODH10\%1m } \\ \text { Channel } & \text { 2487Channel 1 } & \text { Date Processed } & \text { 9/2/2004 11:03:33 AM } \\ \text { Run Time } & \text { 240.0 Minutes } & & \end{array}$

Auto-Scaled Chromatogram

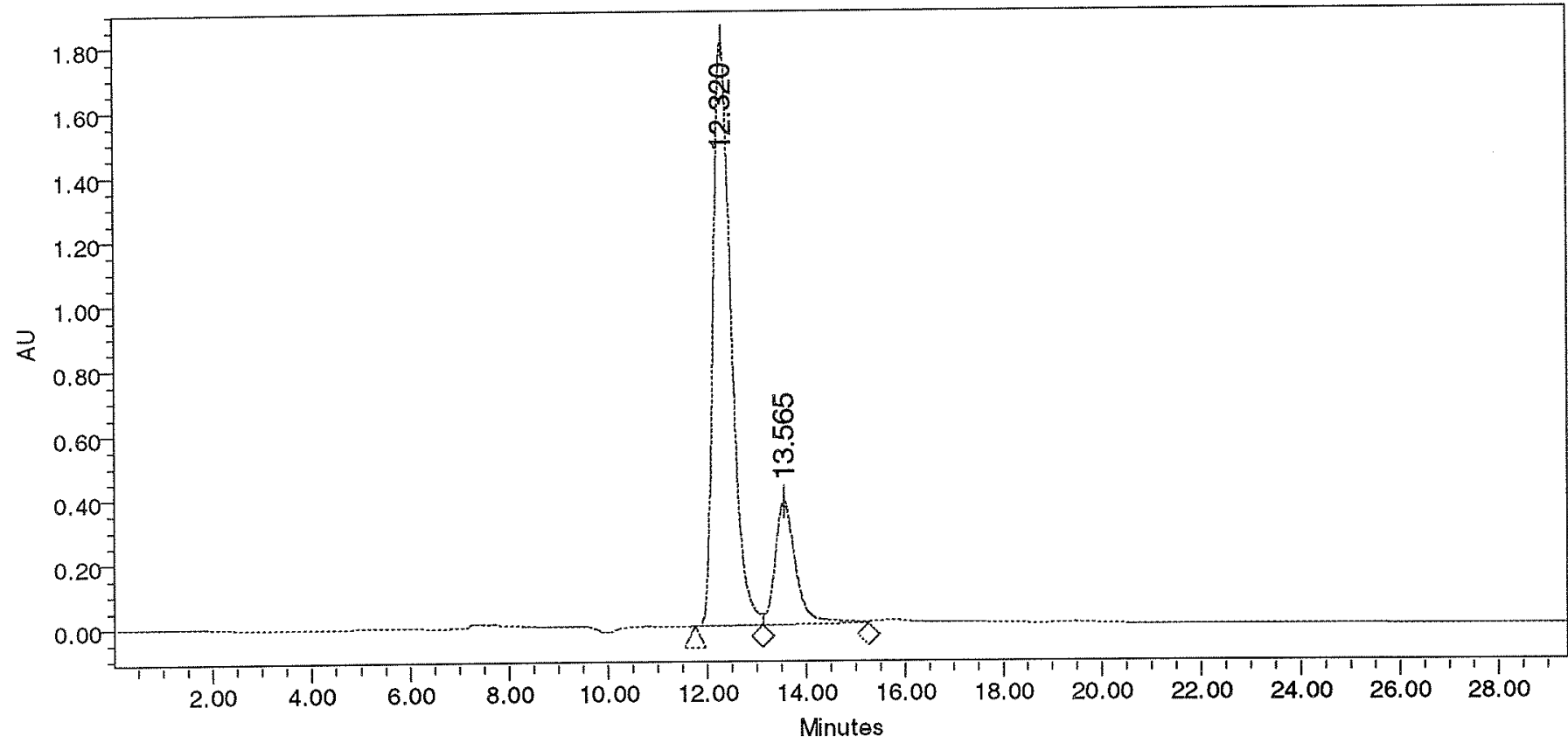

Peak Results

\begin{tabular}{|c|c|c|c|c|}
\hline & Name & RT & Area & $\%$ Area \\
\hline 1 & & 12.320 & 45676348 & 80.36 \\
\hline 2 & & 13.565 & 11165666 & 19.64 \\
\hline
\end{tabular}

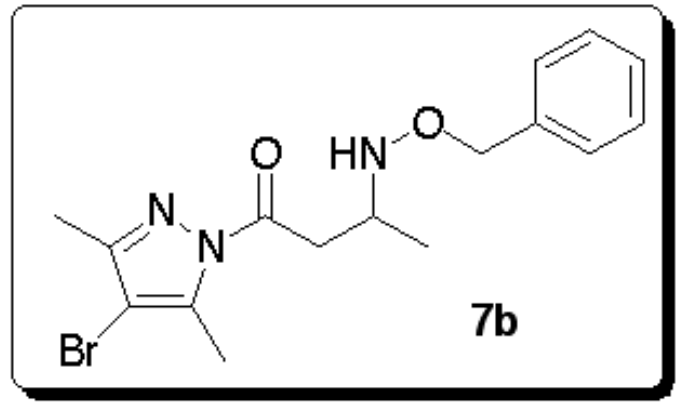



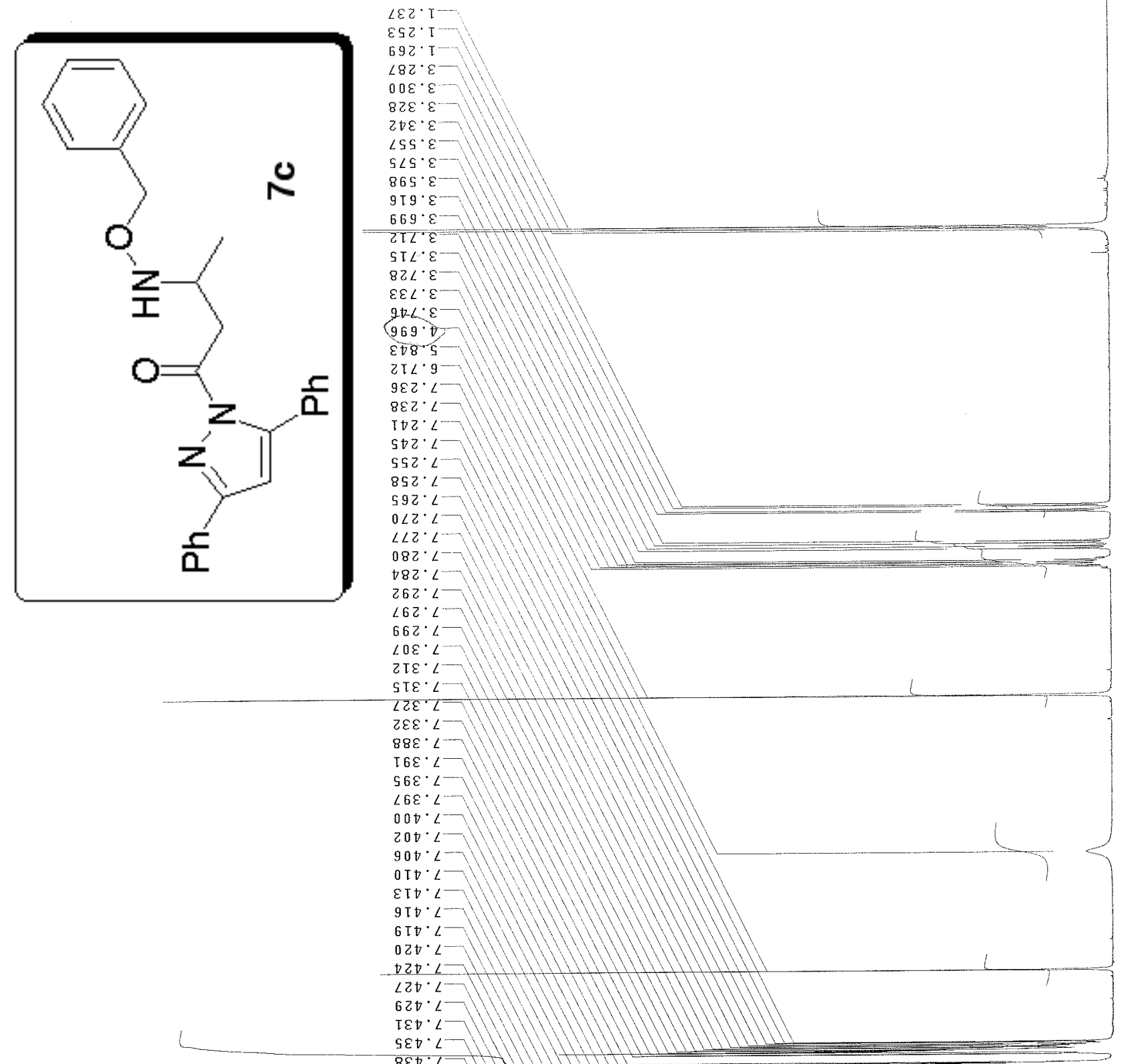

$-1$ में

$\sim$

$m$

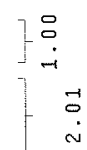

$+$

a
i

เn

0 $] \begin{aligned} & \infty \\ & ? \\ & 0\end{aligned}$

\%े

$N$

]$_{-1}^{0}$

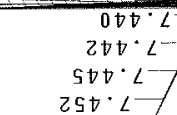

$000 \cdot 2$

$250 .<7$

950 09

$\varepsilon \angle t<$

$\angle \angle 0^{\circ} \angle-$

$280^{\circ} L$

โ $68^{\circ} \angle$

$768^{\circ} \angle$

$868^{\circ} \mathrm{L}$

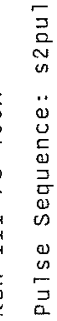

II $6^{\circ}$

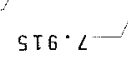

$\infty\rfloor \stackrel{0}{\stackrel{c}{0}}$

$a$

먹 

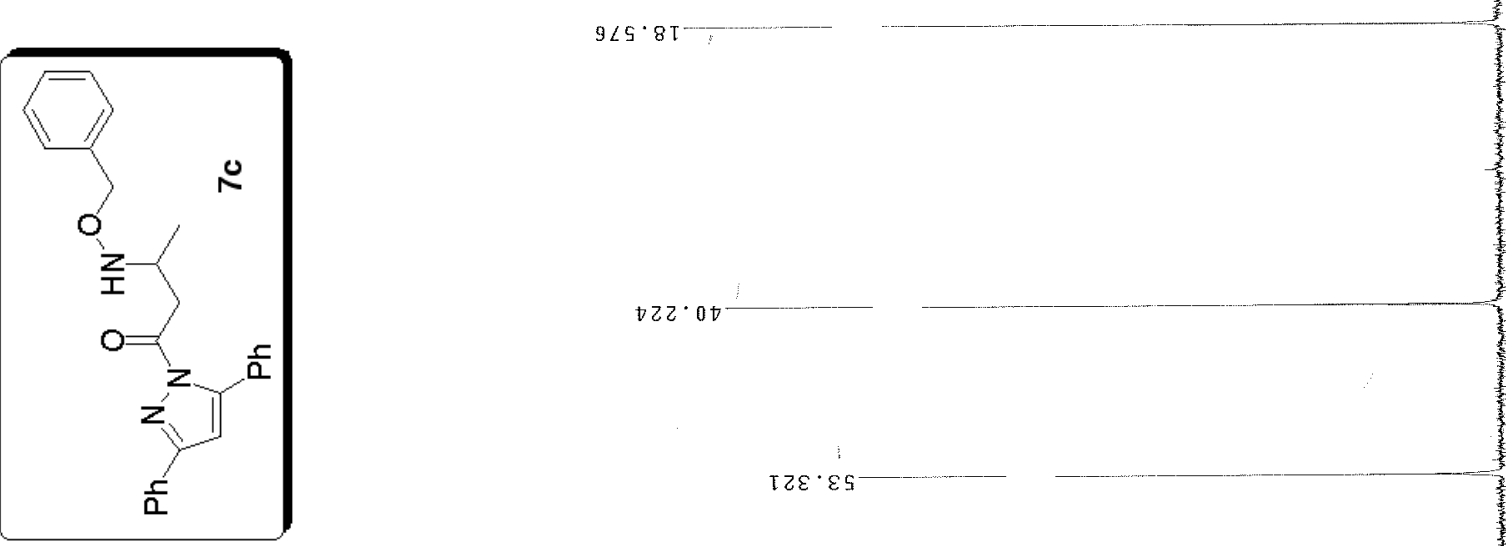

$2 \angle 8^{\circ} 9 \angle \angle$ $\varepsilon 56.9 L$ $\varepsilon 92 \cdot \angle L$ $\angle 85^{\circ} \angle L^{\circ}$

$680^{\circ} 0 \mathrm{~T}$

$560^{\circ} 92 \mathrm{I}$

$156^{\circ} \angle Z T$

Q-1- 82 $8598 \mathrm{ZT}$

$066^{\circ} 82 \mathrm{I}^{-\cdots}$

$26 T^{\circ} 62 \mathrm{~T}$ $807.62 T$

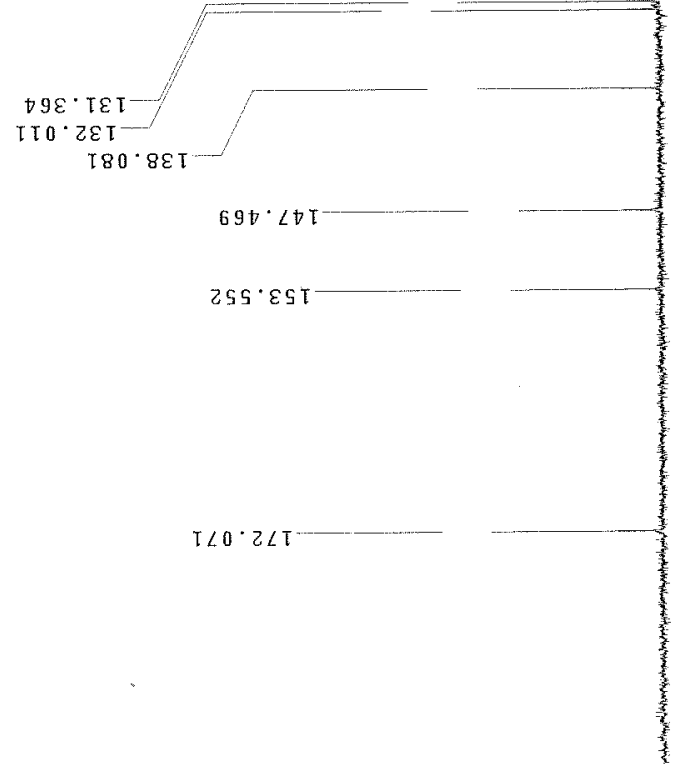




\section{Sample Information}

$\begin{array}{llll}\text { SampleName } & \text { KII547AD10\%1m } & \text { Sample Type } & \text { Unknown } \\ \text { Vial } & 1 & \text { Date Acquired } & \text { 9/4/2004 10:41:41 AM } \\ \text { Injection } & 1 & \text { Acq Method Set } & \text { 10\%iPOH 1mpm } \\ \text { Injection Volume } & 10.00 \text { ul } & \text { Processing Method Kll547AD10\%1m } \\ \text { Channel } & \text { 2487Channel 1 } & \text { Date Processed } & \text { 9/4/2004 11:48:18 AM } \\ \text { Run Time } & \text { 240.0 Minutes } & & \end{array}$

Auto-Scaled Chromatogram

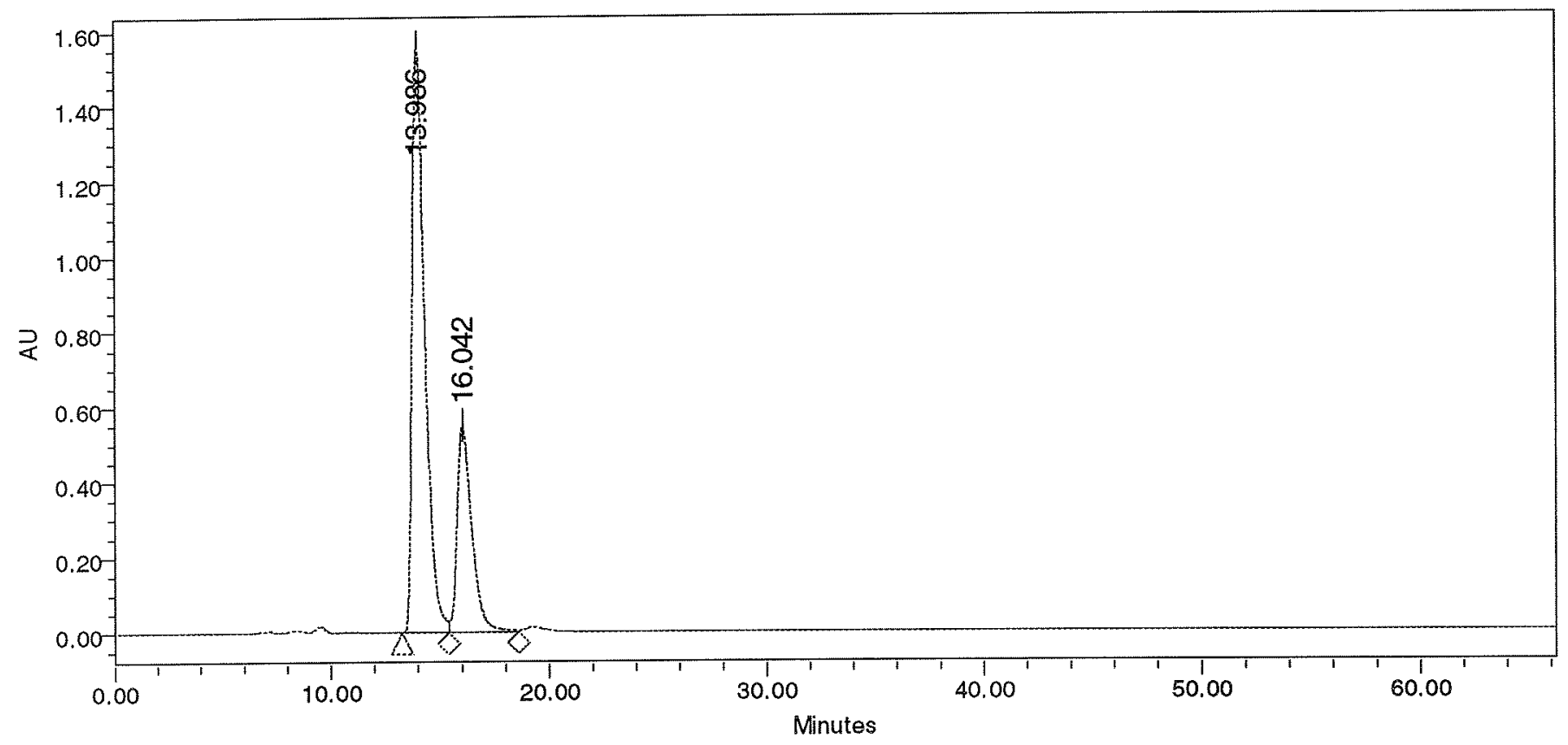

Peak Results
\begin{tabular}{|r|c|c|c|r|}
\hline & Name & RT & Area & $\%$ Area \\
\hline 1 & & 13.986 & 62704242 & 70.95 \\
\hline 2 & & 16.042 & 25679487 & 29.05 \\
\hline
\end{tabular}

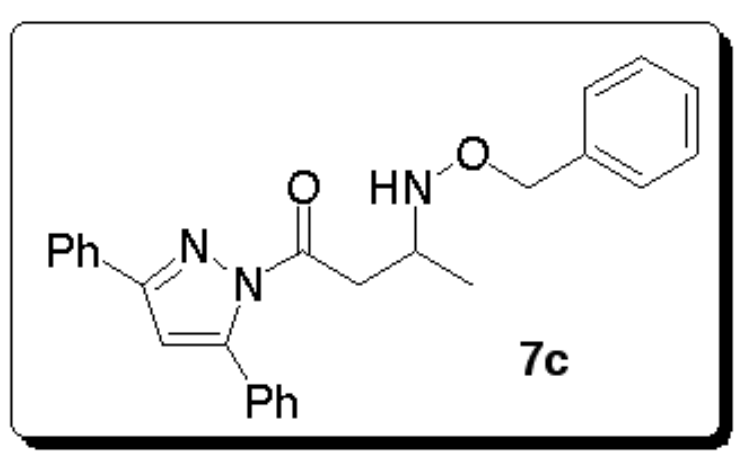




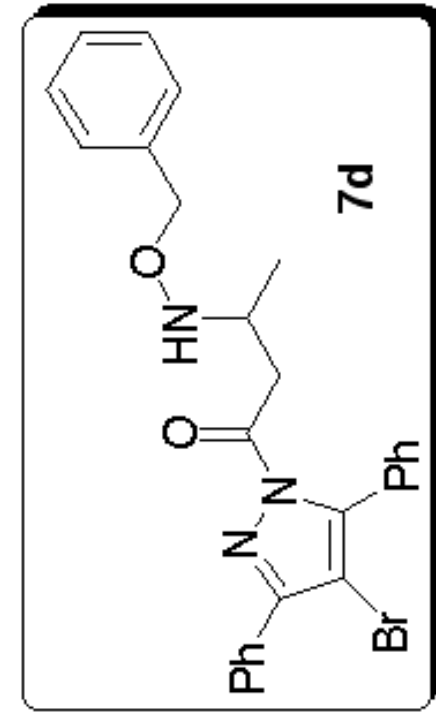

$\frac{E}{2}$

005.1

$215^{\circ}$

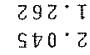

$\angle 02 \cdot \varepsilon^{\circ}$

$022 \cdot \varepsilon$

$8+2 \cdot \varepsilon$

$905 \cdot 8$

$525 \cdot \varepsilon$

$\angle D S^{\circ} \varepsilon$

$999^{\circ} \varepsilon$

$259^{\circ} \varepsilon$

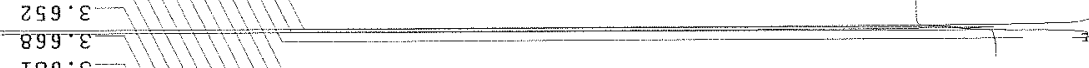

$189^{\circ} \varepsilon^{-}$

$\angle 69 \cdot \varepsilon$

$\varepsilon 0 L^{\circ} \varepsilon^{\prime}$

SIL.

$\frac{199 \cdot t}{\varepsilon 6 \angle \cdot S}$

$5 \varepsilon 2$

$\varepsilon \triangleright z^{\prime} \iota$

$\angle t Z \cdot \angle$

$152 \cdot 2$

$852^{\circ} \angle$

$8 \angle 2^{\circ} \angle$

$882^{\circ}:$

$662^{\circ} \mathrm{L}$

$208^{\circ}$

I $1 \varepsilon \cdot 2$

$\angle \mathrm{T} \varepsilon \cdot \angle-$

T $2 \varepsilon^{\circ} L$

$\varepsilon 2 \varepsilon \cdot L$

$9 z \varepsilon \cdot\llcorner$

$\downarrow \varepsilon \varepsilon^{\circ}$

$8 \varepsilon \varepsilon^{\circ}$

$s+\varepsilon \cdot<-$

$80 \varepsilon^{\circ} \angle$

TรE. $\angle$

SSE 2

$85 \varepsilon^{\circ} /$

$\varepsilon 9 \varepsilon^{\circ} \angle$

$99 \varepsilon \cdot<-$

$698 \cdot<$

$T D D^{\circ} \angle$

$900 \cdot$

ost 2 -

SSt. $\angle$

$850^{\circ}<$
$T 90^{\circ}<$

990.

$\mathrm{r} \angle \mathrm{t} \cdot \mathrm{L}$

$180^{\circ}<$

$580^{\circ}$

5602.2
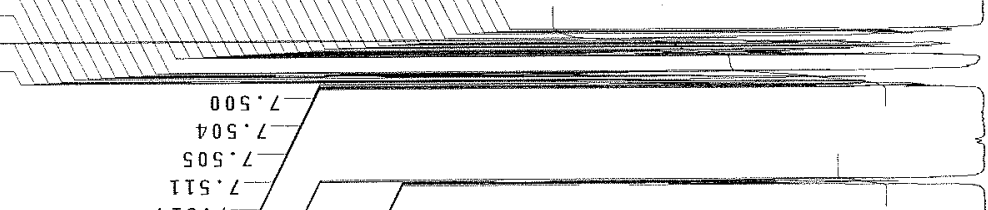

t $\mathrm{TS}^{\circ} \angle$

$815^{\circ} L$

$225^{\circ}$
$925^{\circ}$

$\varepsilon \angle G^{\circ} \angle$

$086^{\circ} \mathrm{L}$
$+86^{\circ} \mathrm{L}$

$786^{\circ} \mathrm{L}$

$\angle 86 \cdot 1$

$686^{\circ} \mathrm{L}$

$\$ 66^{\circ}<$

$\angle 66^{\circ} \angle$

$666^{\circ} \mathrm{L}$

i

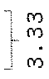

$\sim$

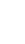

$m$

$+$

$\overrightarrow{\mathrm{n}}$

เ

-

$\infty] \stackrel{\sim}{\circ}$

$\sigma$ 

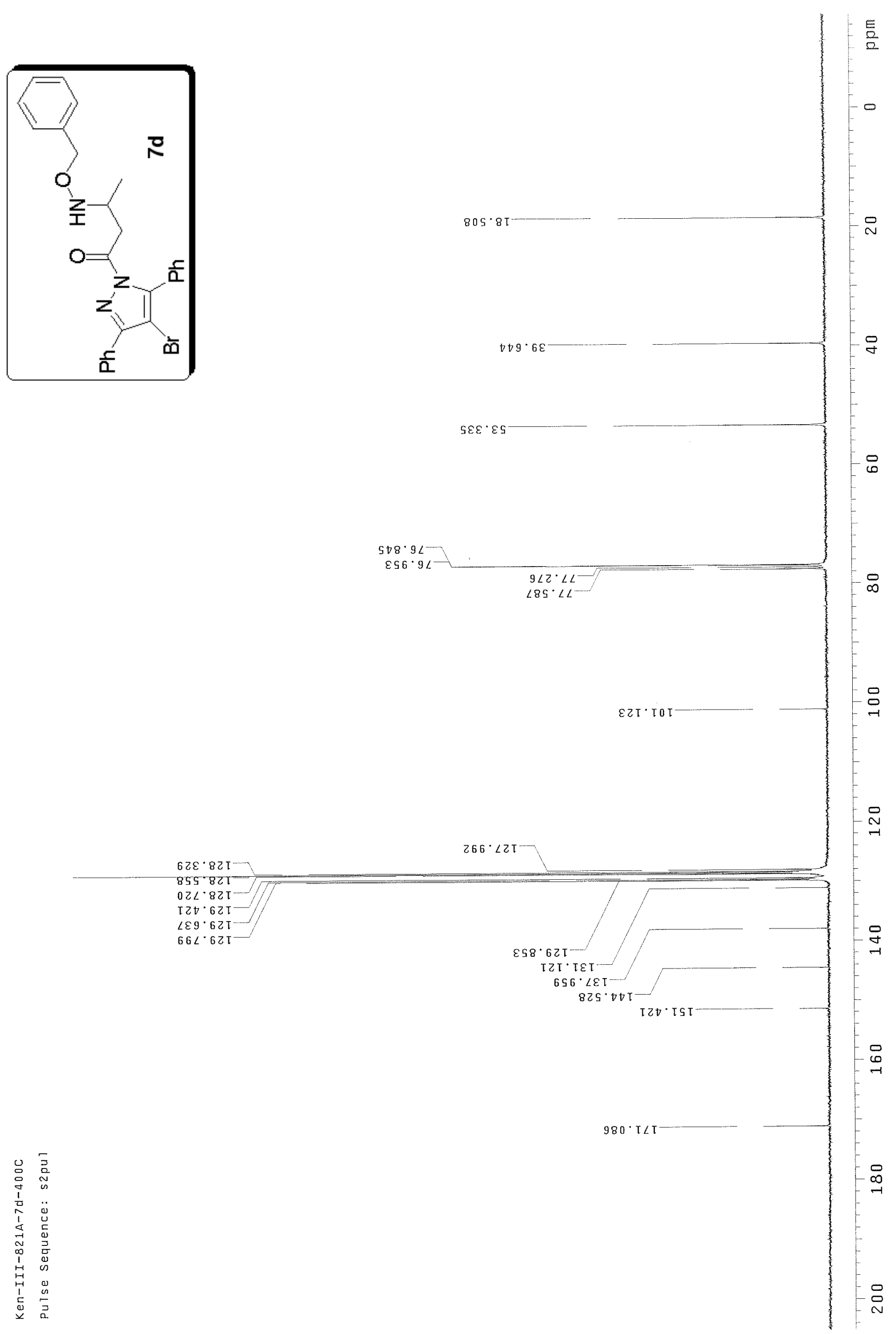


\section{Sample Information}

$\begin{array}{llll}\text { SampleName } & \text { Kll554OJOJ20\%1m } & \text { Sample Type } & \text { Unknown } \\ \text { Vial } & 1 & \text { Date Acquired } & \text { 9/10/2004 10:17:15 AM } \\ \text { Injection } & 2 & \text { Acq Method Set } & \text { 20\%iPOH 1mpm } \\ \text { Injection Volume } & 20.00 \mathrm{ul} & \text { Processing Method Kll554OJOJ20\%1m } \\ \text { Channel } & \text { 2487Channel 1 } & \text { Date Processed } & \text { 9/10/2004 1:53:53 PM } \\ \text { Run Time } & \text { 240.0 Minutes } & & \end{array}$

\section{Auto-Scaled Chromatogram}

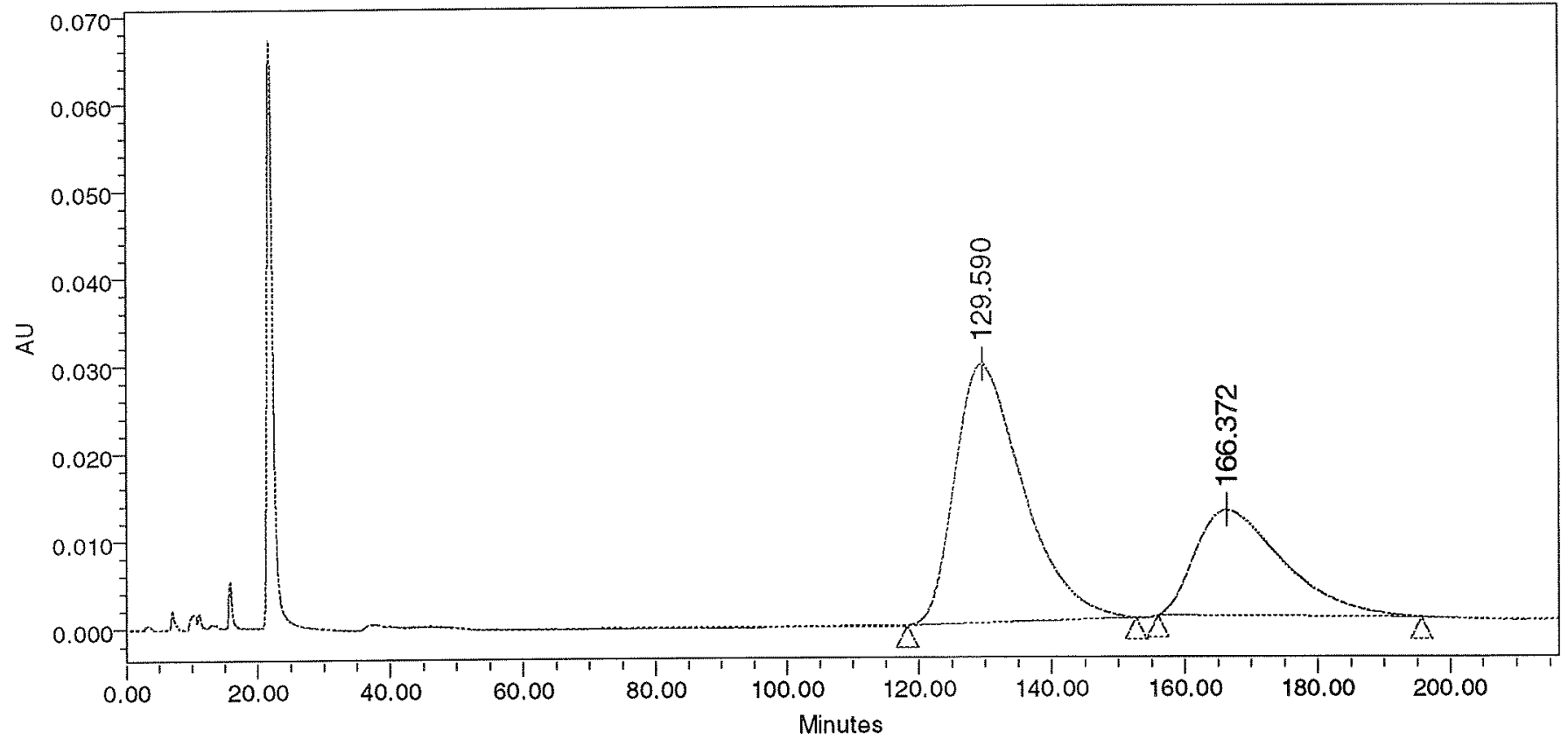

Peak Results
\begin{tabular}{|r|c|c|c|r|}
\hline & Name & RT & Area & $\%$ Area \\
\hline 1 & & 129.590 & 21021500 & 65.71 \\
\hline 2 & & 166.372 & 10971673 & 34.29 \\
\hline
\end{tabular}<smiles>CC(CC(=O)n1nc(-c2ccccc2)c(Br)c1-c1ccccc1)NOCc1ccccc1</smiles> 

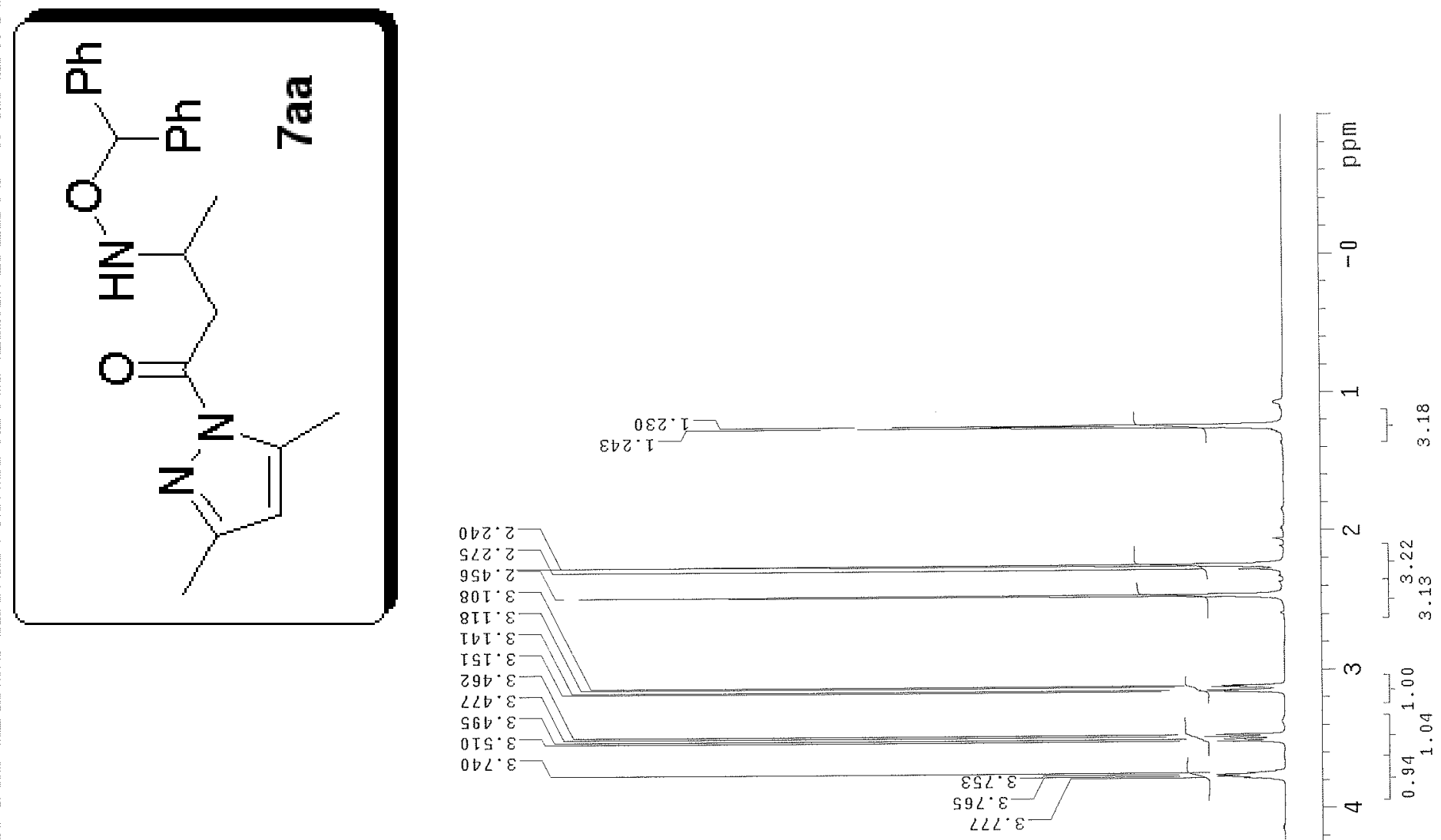

E

1

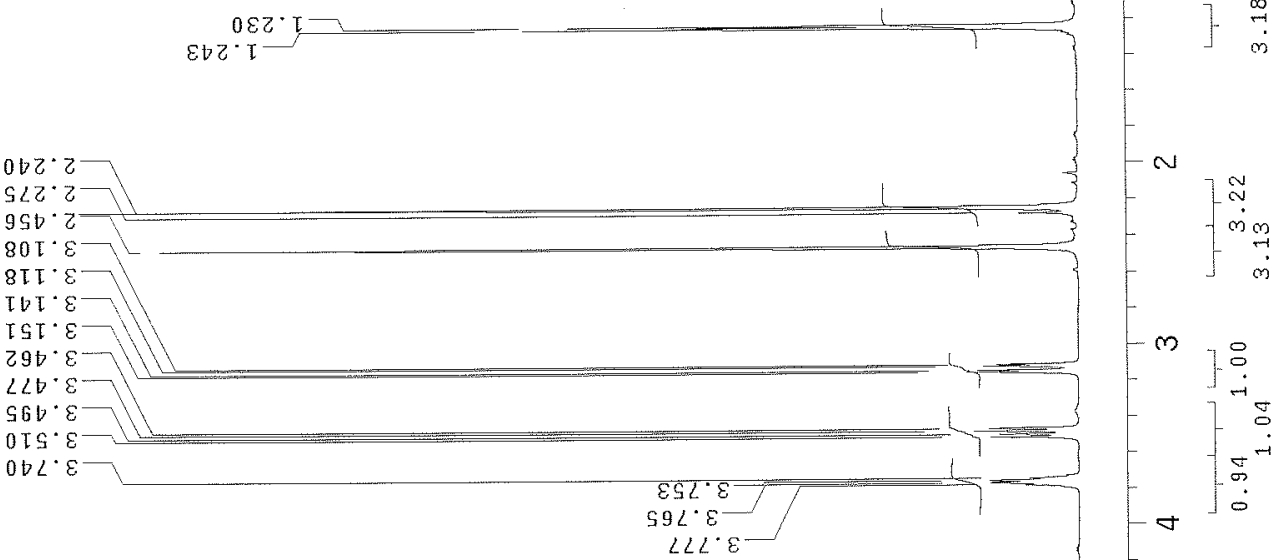

$-r$

$\stackrel{\infty}{\stackrel{\infty}{\hookrightarrow}}$

$-N$

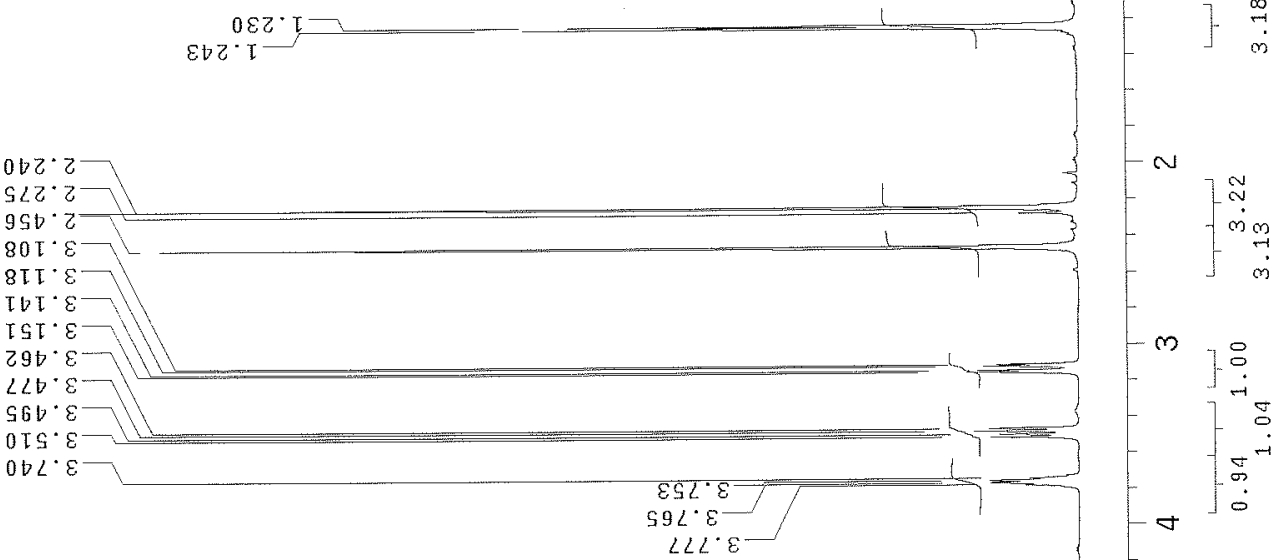

พั

$969^{\circ}$.

$088^{\circ}$

ES8.5

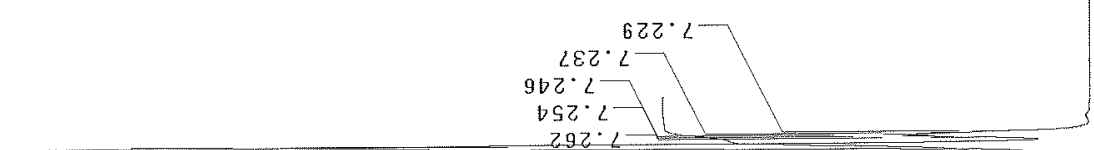

$562 \cdot 2$

So $\varepsilon^{\circ} \angle$

STE. 2
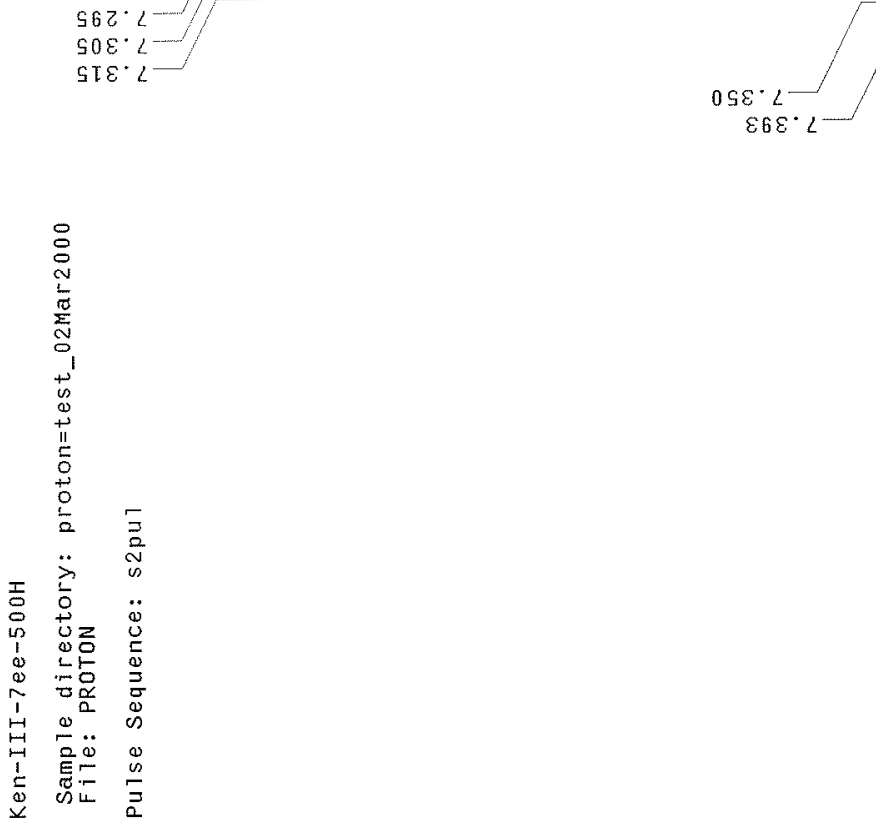

$\infty$ 


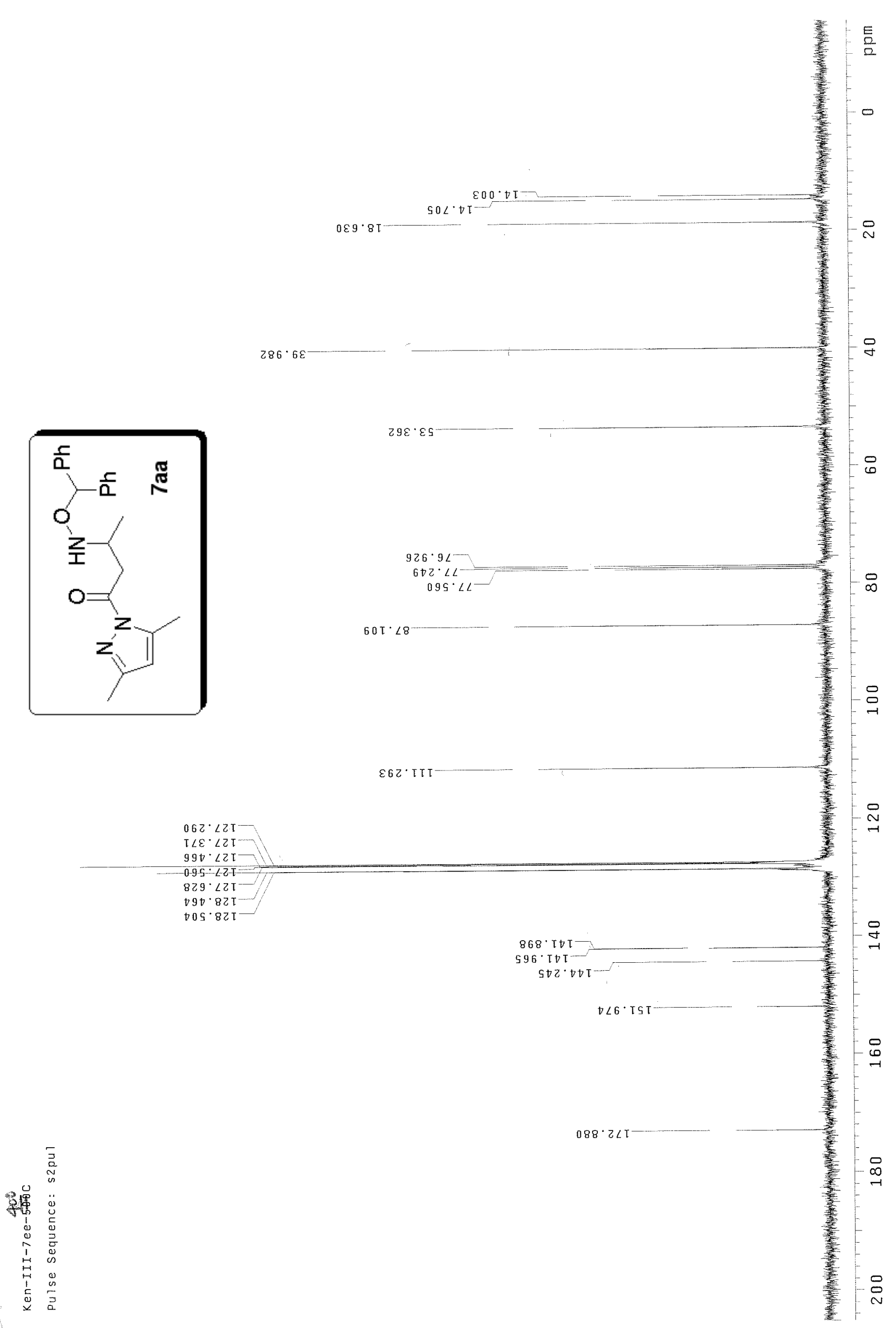




\section{Sample Information}

SampleName KIII1760ODHpi\%05m

Vial 1

Injection 1

Injection Volume $20.00 \mathrm{ul}$

Channel 2487Channel 1

Run Time 240.0 Minutes
Sample Type Unknown

Date Acquired $\quad$ 12/23/2004 1:15:11 PM

Acq Method Set $\quad 1 \%$ iPrOH 05mpm

Processing Method KIIII7600DHp1\%05m

Date Processed 12/23/2004 3:10:21 PM

Auto-Scaled Chromatogram

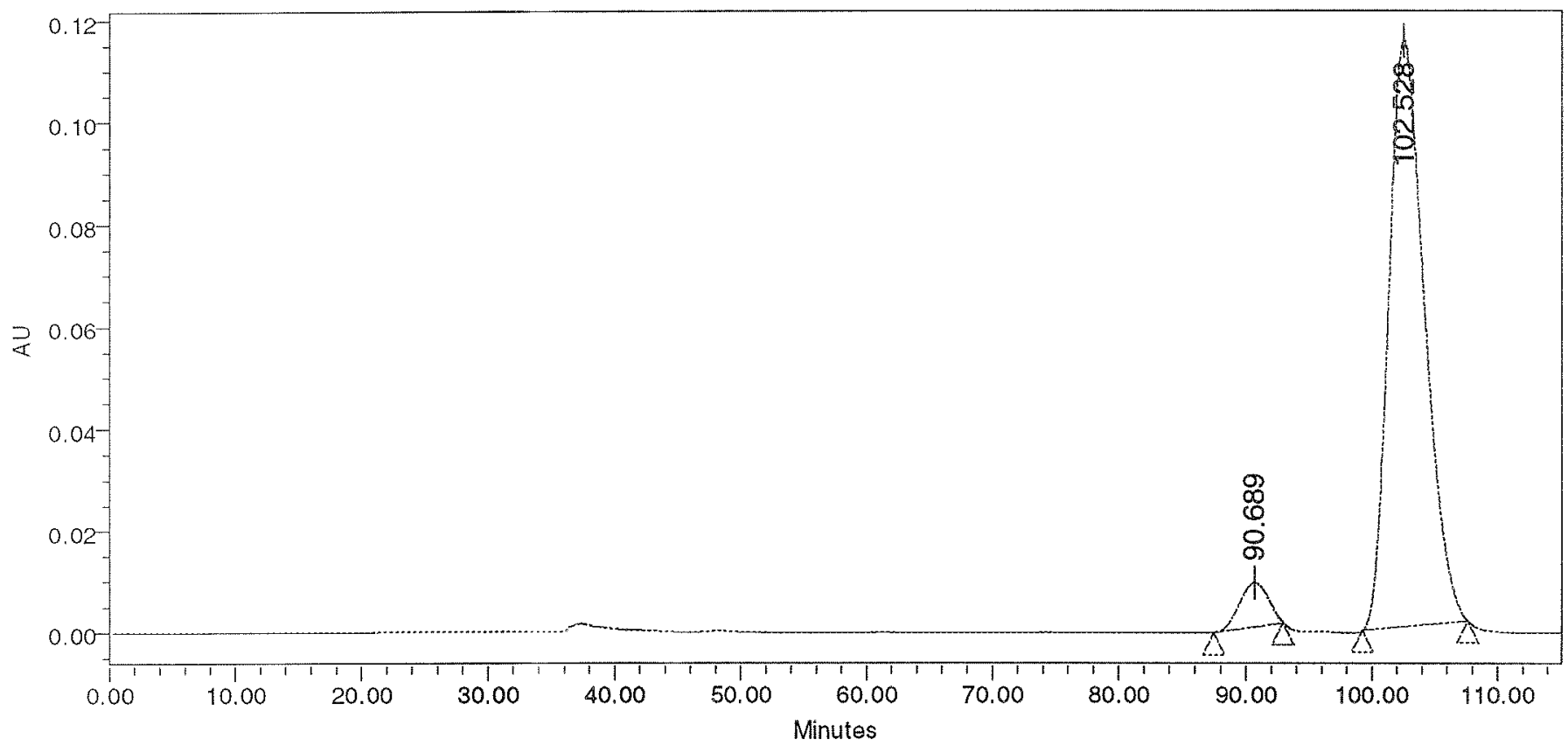

Peak Results

\begin{tabular}{|c|c|c|c|c|}
\hline & Name & RT & Area & $\%$ Area \\
\hline 1 & & 90.689 & 1365567 & 5.62 \\
\hline 2 & & 102.528 & 22932077 & 94.38 \\
\hline
\end{tabular}

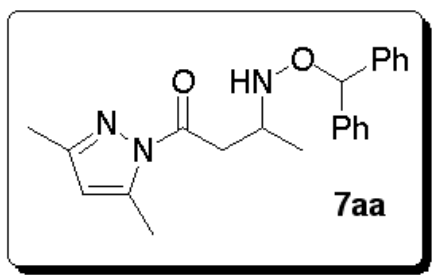



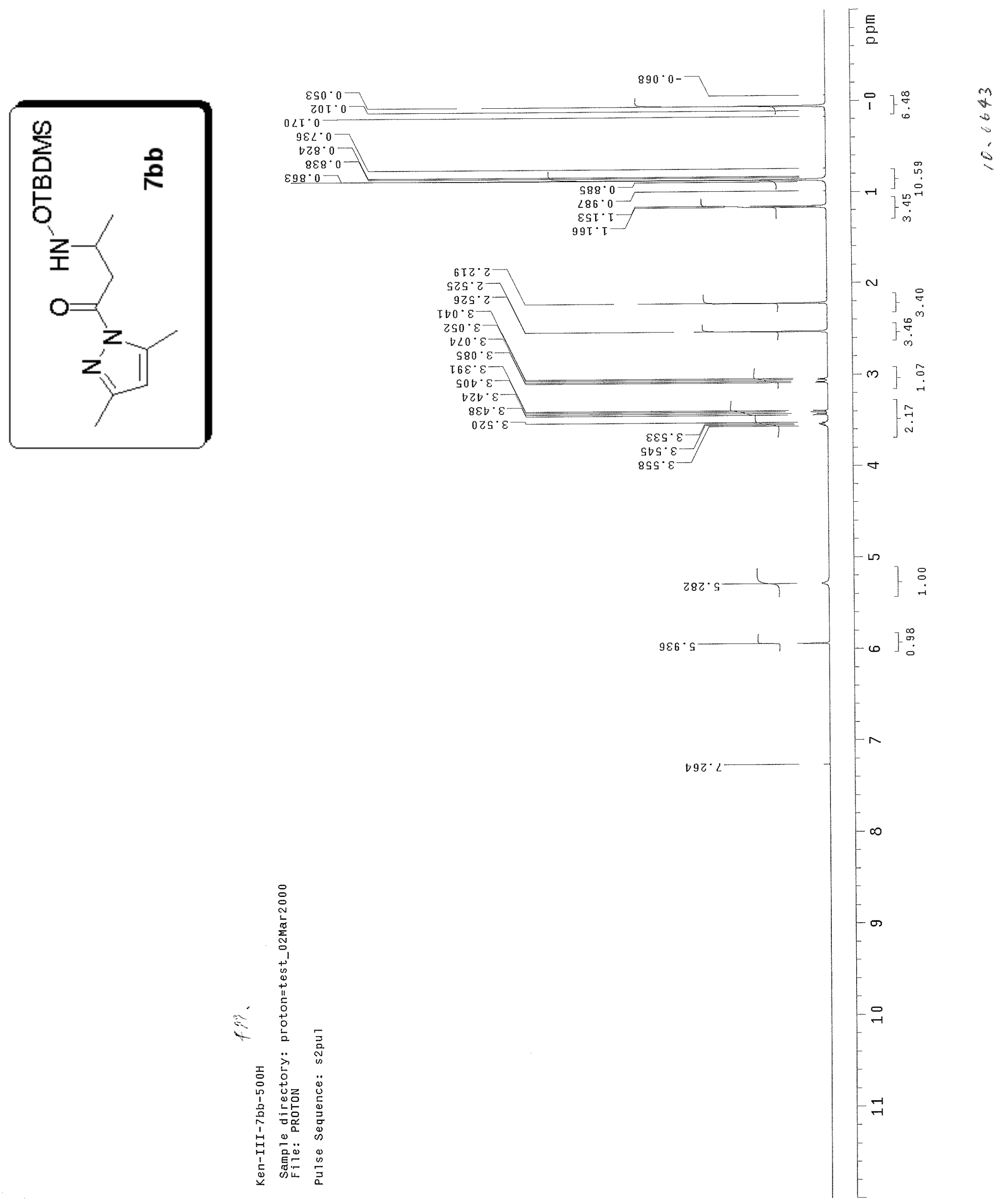


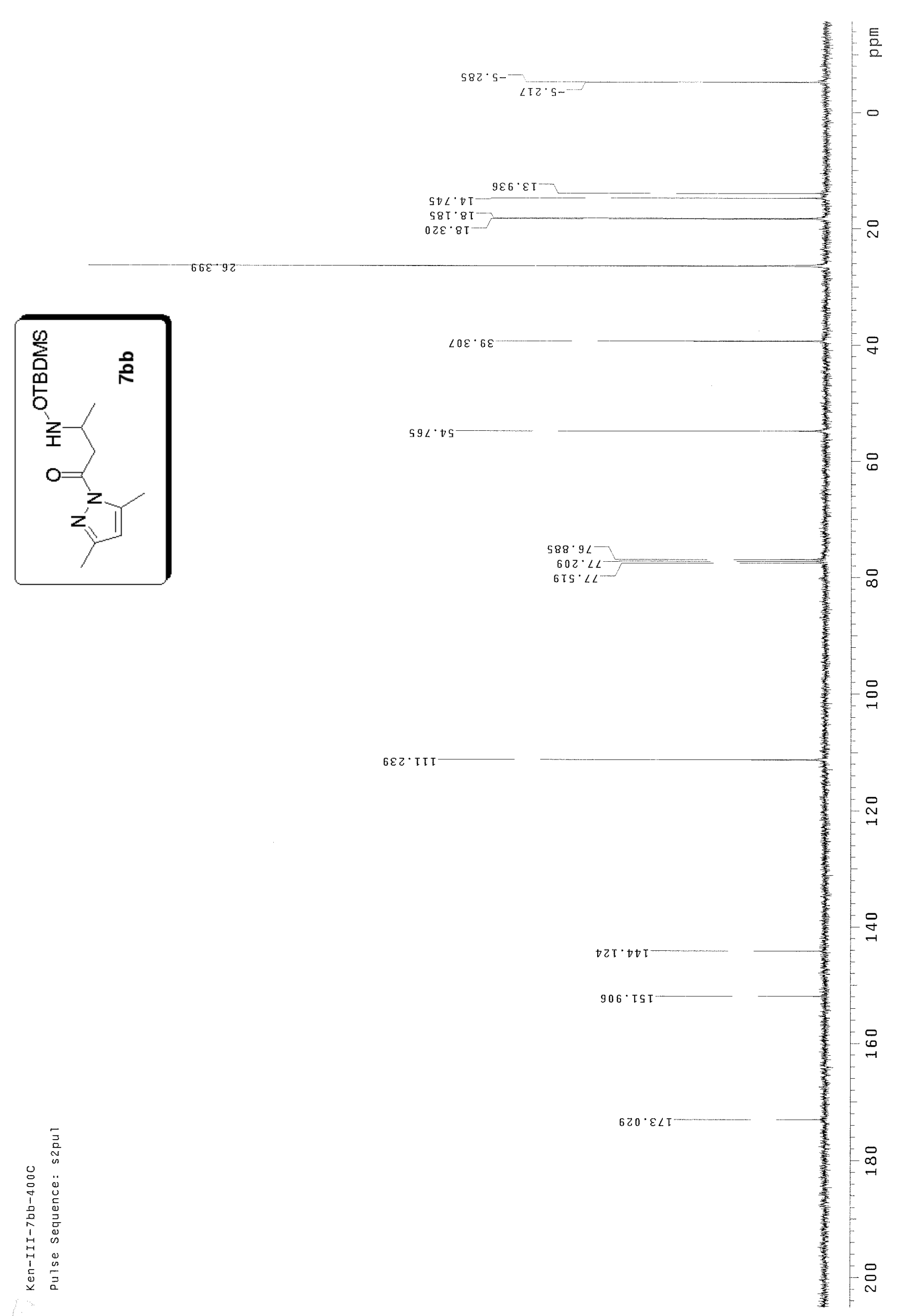

圈 


\section{Sample Information}

$\begin{array}{llll}\text { SampleName } & \text { Kill6910J0J1\%05ml } & \text { Sample Type } & \text { Unknown } \\ \text { Vial } & 1 & \text { Date.Acquired } & \text { 11/12/2004 6:06:28 PM } \\ \text { Injection } & 1 & \text { Acq Method Set } & \text { 1\%iPrOH 05mpm } \\ \text { Injection Volume } & 20.00 \mathrm{ul} & \text { Processing Method KIll/6910JOJ1\%05m } \\ \text { Channel } & \text { 2487Channel 1 } & \text { Date Processed } & \text { 11/12/2004 8:04:00 PM } \\ \text { Run Time } & \text { 240.0 Minutes } & & \end{array}$

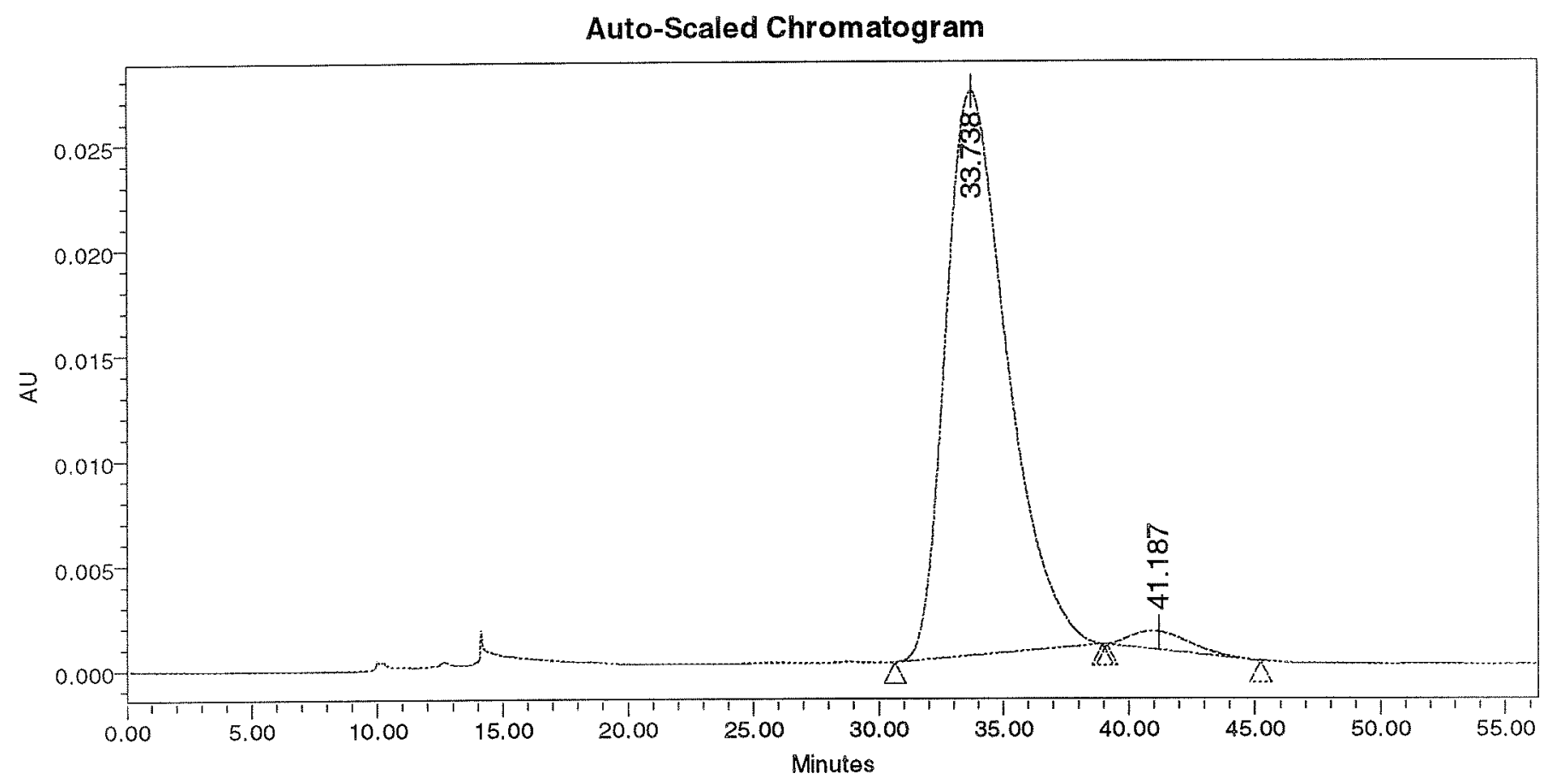

\section{Peak Results}

\begin{tabular}{|c|c|c|c|r|}
\hline & Name & RT & Area & $\%$ Area \\
\hline 1 & & 33.738 & 4630546 & 97.11 \\
\hline 2 & & 41.187 & 138013 & 2.89 \\
\hline
\end{tabular}

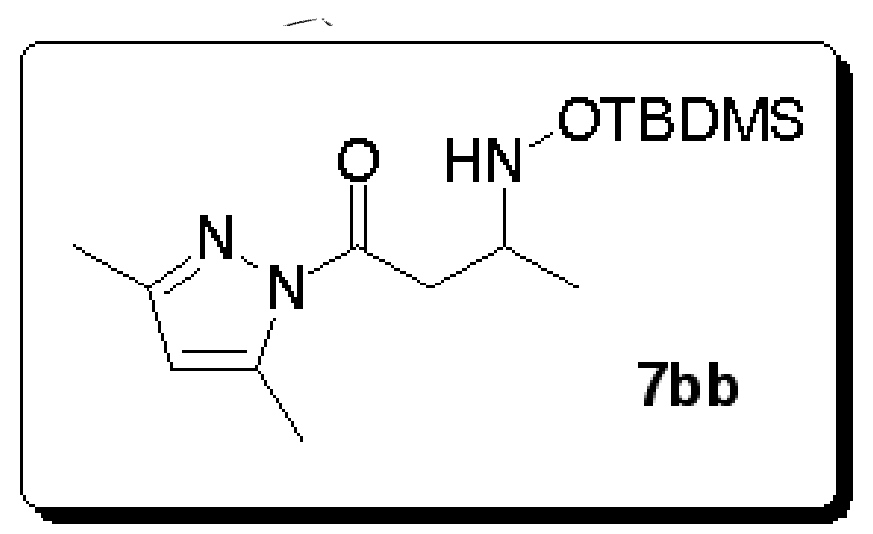



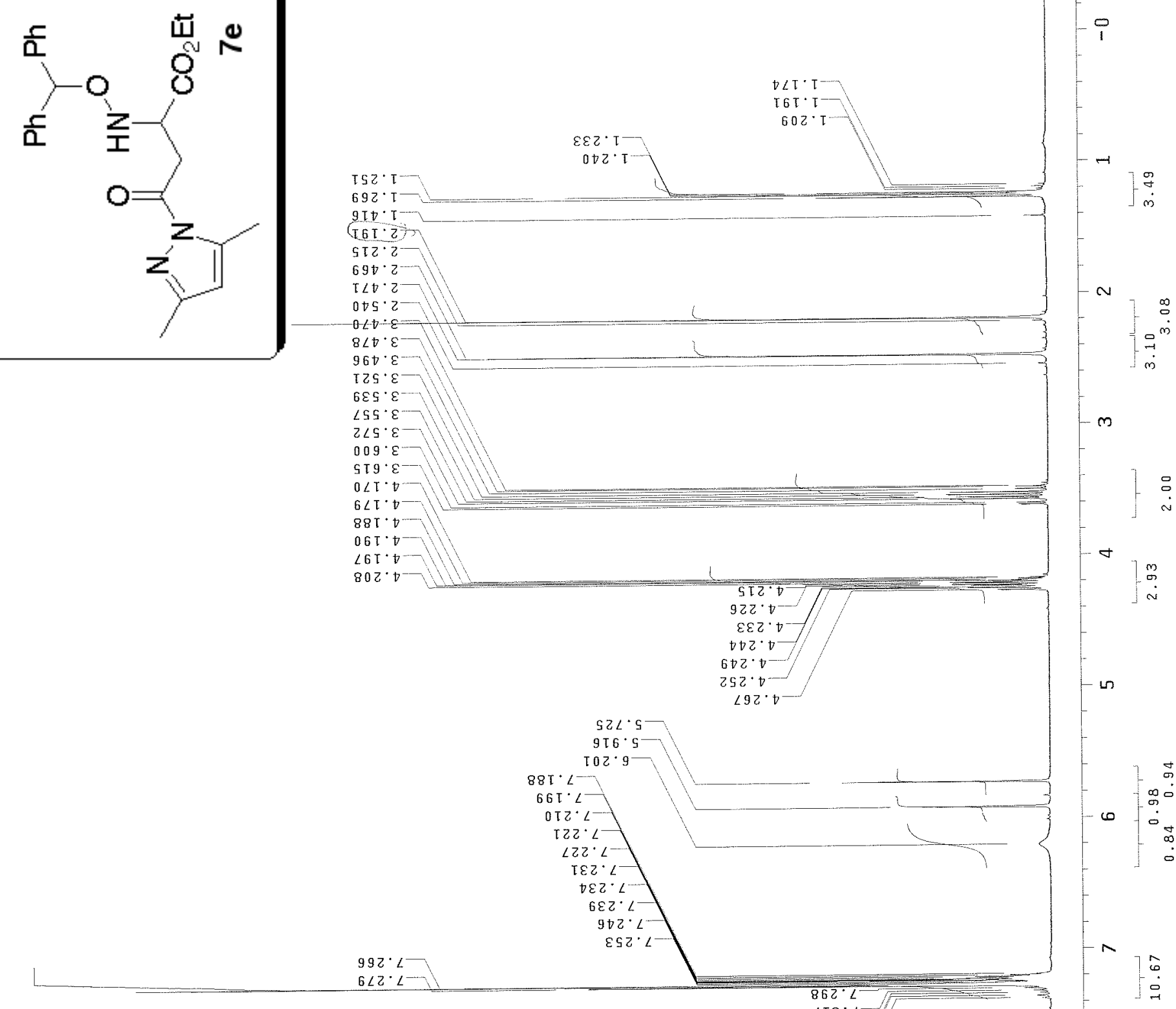

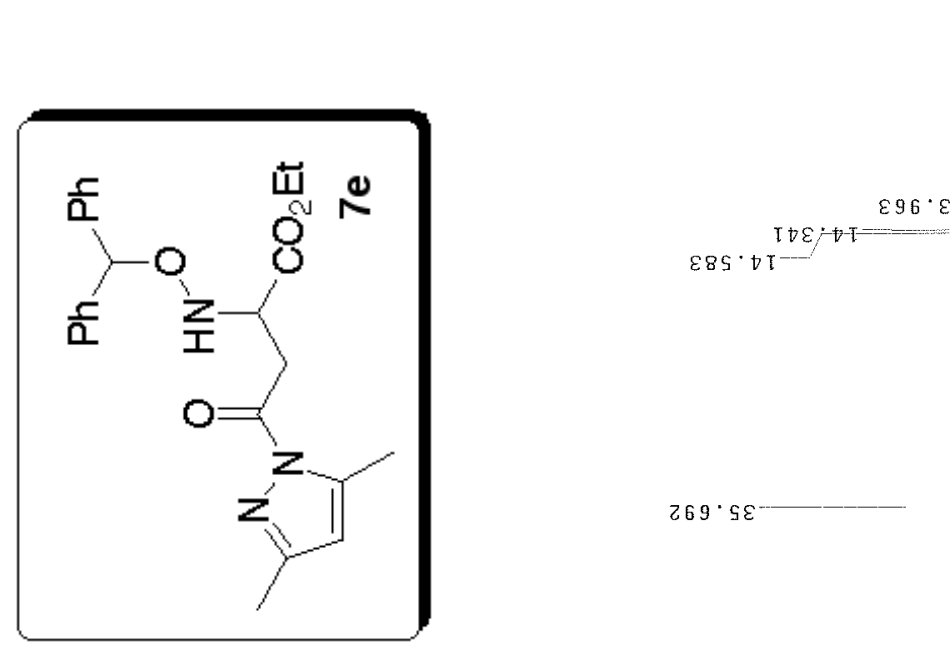

$668 \cdot 9 L$

$\varepsilon \varepsilon \mathrm{S} \cdot \angle L$

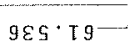

옹

796.98

$90 S^{\circ} \angle Z T$

$89.2 \div 2-1$

$801^{\circ} \angle Z T$

$057 \cdot 82 \mathrm{~T}$

$8 z t \cdot \operatorname{tr}$

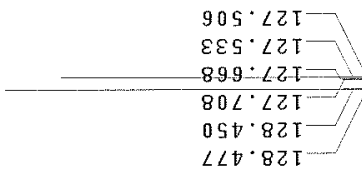

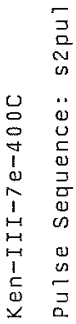

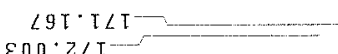




\section{Sample Information}

$\begin{array}{llll}\text { SampleName } & \text { KIIII762ADHp20\%1m } & \text { Sample Type } & \text { Unknown } \\ \text { Vial } & 1 & \text { Date Acquired } & \text { 12/23/2004 12:42:18 PM } \\ \text { Injection } & 1 & \text { Acq Method Set } & \text { 20\%iPrOH 1mpm } \\ \text { Injection Volume } & 20.00 \mathrm{ul} & \text { Processing Method KIIII762ADHp20\%1m } \\ \text { Channel } & \text { 2487Channel 1 } & \text { Date Processed } & \text { 12/23/2004 1:31:49 PM } \\ \text { Run Time } & \text { 240.0 Minutes } & & \end{array}$

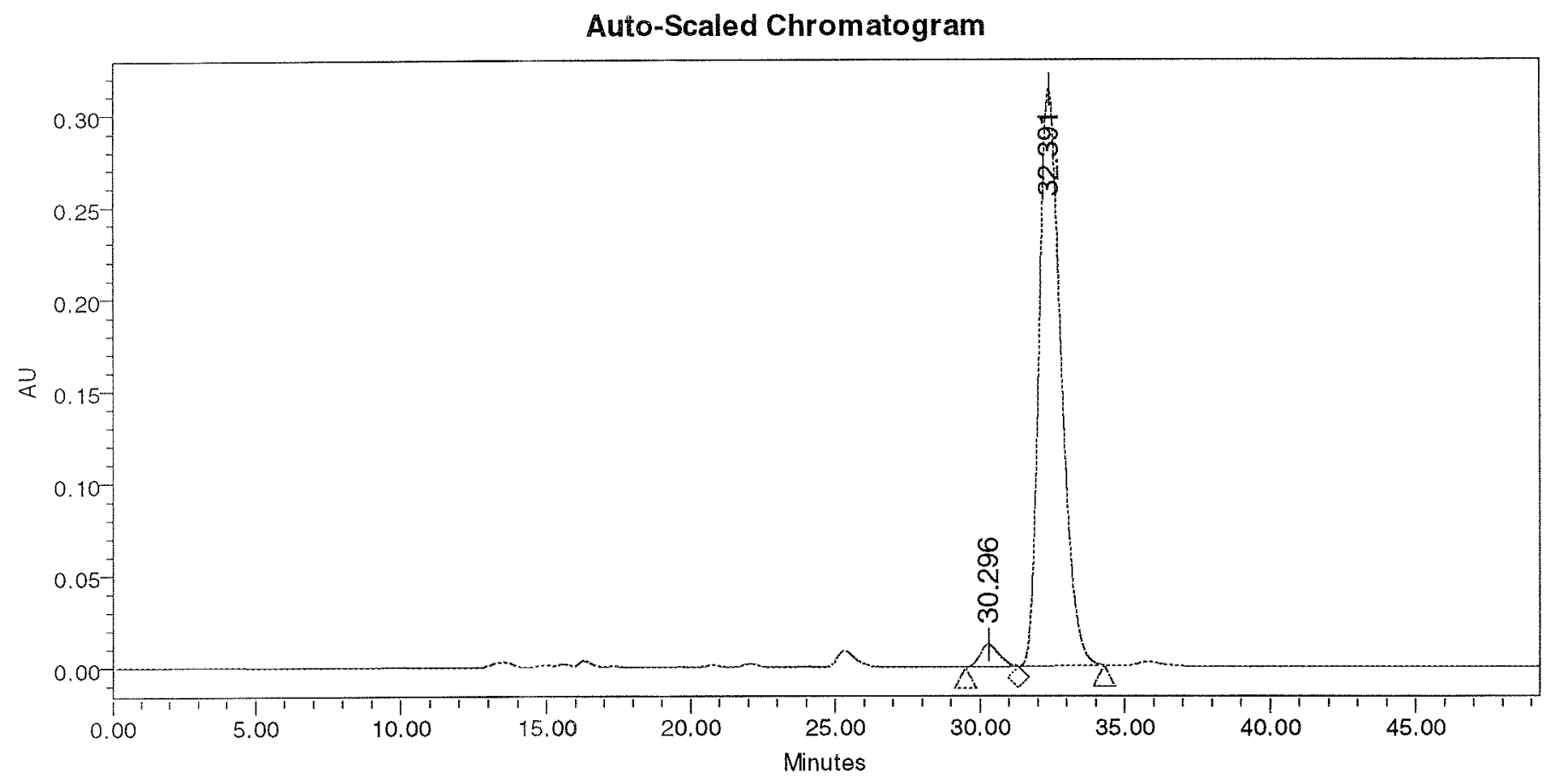

Peak Results

\begin{tabular}{|c|c|c|c|r|}
\hline & Name & RT & Area & $\%$ Area \\
\hline 1 & & 30.296 & 557179 & 3.25 \\
\hline 2 & & 32.391 & 16603257 & 96.75 \\
\hline
\end{tabular}

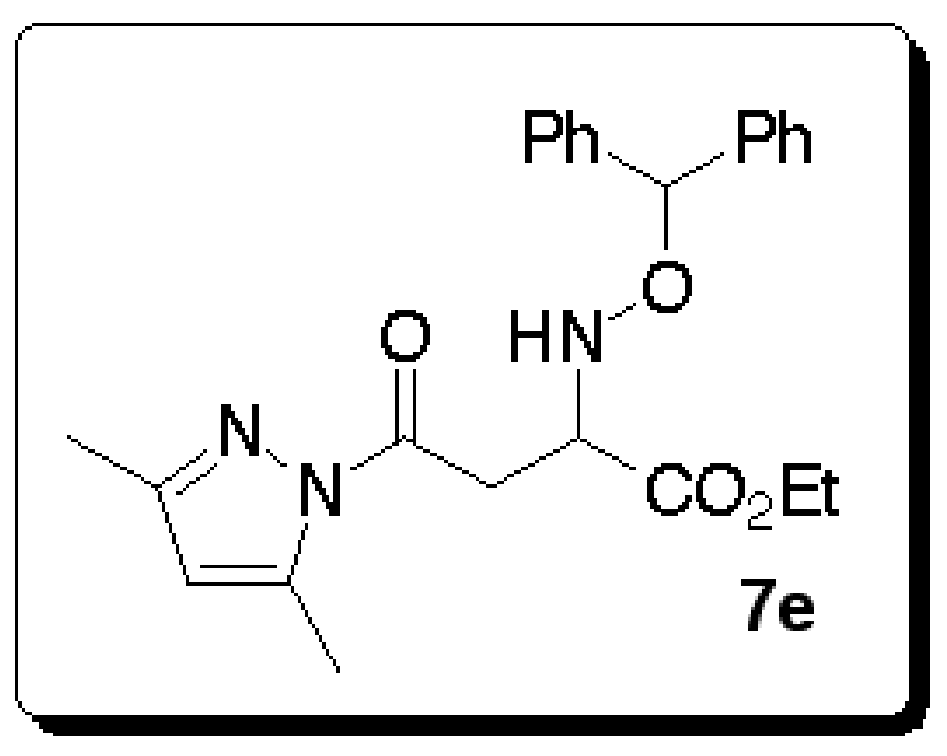




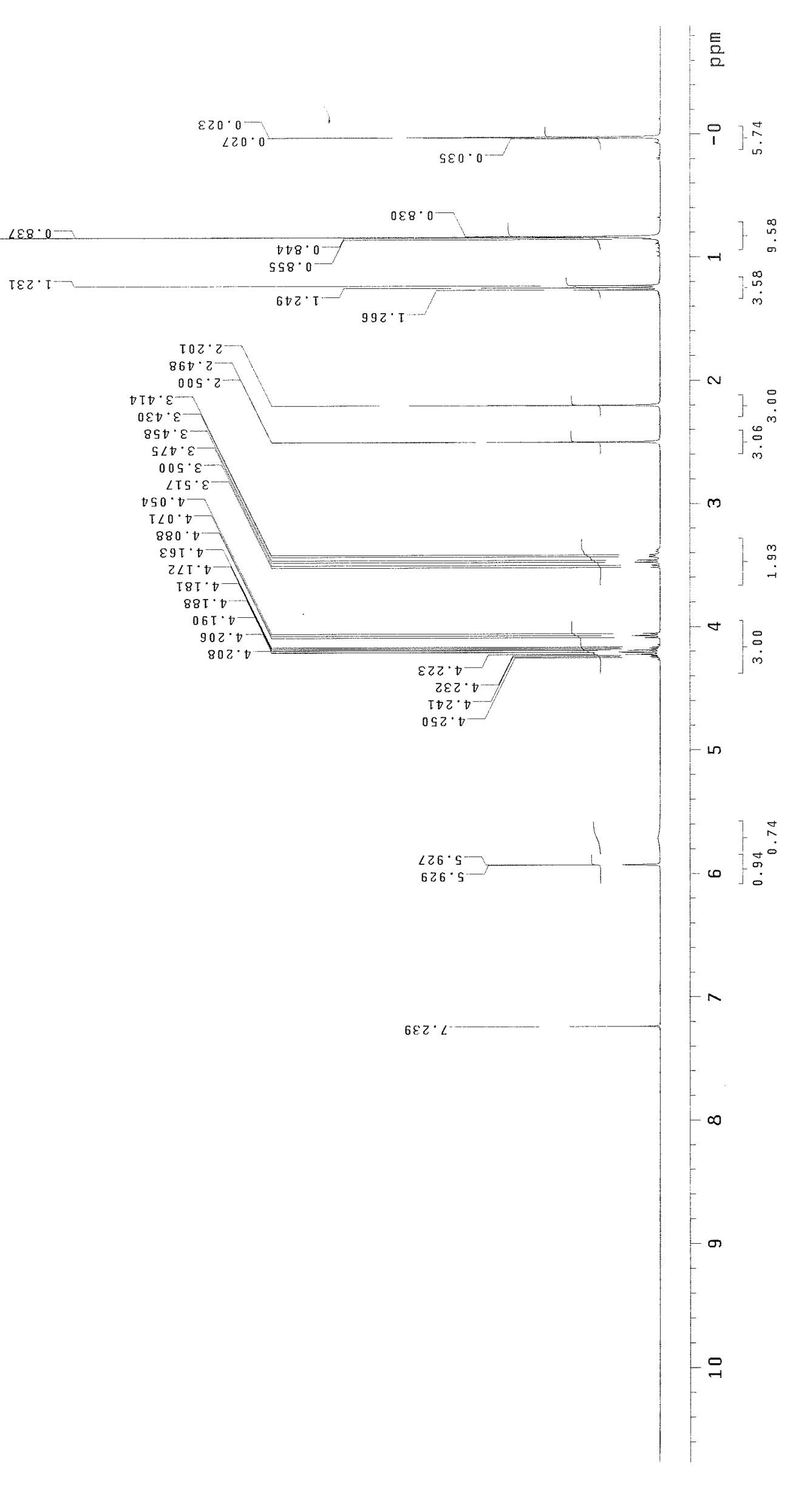




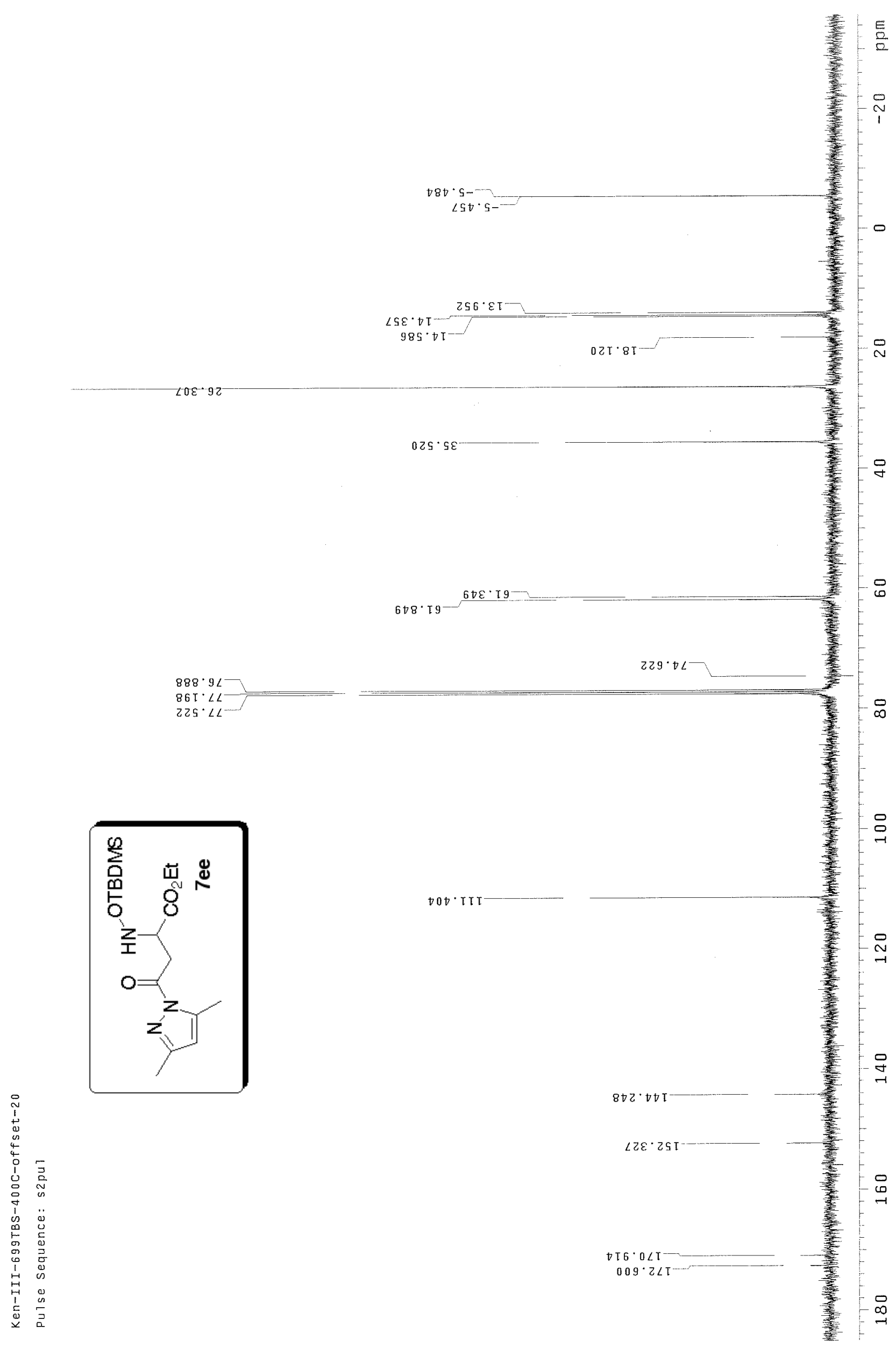




\section{Sample Information}

$\begin{array}{llll}\text { SampleName } & \text { KIII700ODHp1\%05m } & \text { Sample Type } & \text { Unknown } \\ \text { Vial } & 1 & \text { Date Acquired } & \text { 12/2/2004 10:59:48 AM } \\ \text { Injection } & 1 & \text { Acq Method Set } & 1 \% \text { PrOH 05mpm } \\ \text { Injection Volume } & 20.00 \mathrm{ul} & \text { Processing Method KIIII700ODH 1\%05m } \\ \text { Channel } & 2487 \text { Channel 1 } & \text { Date Processed } & \text { 12/2/2004 12:33:28 PM } \\ \text { Run Time } & \text { 240.0 Minutes } & & \end{array}$

Auto-Scaled Chromatogram

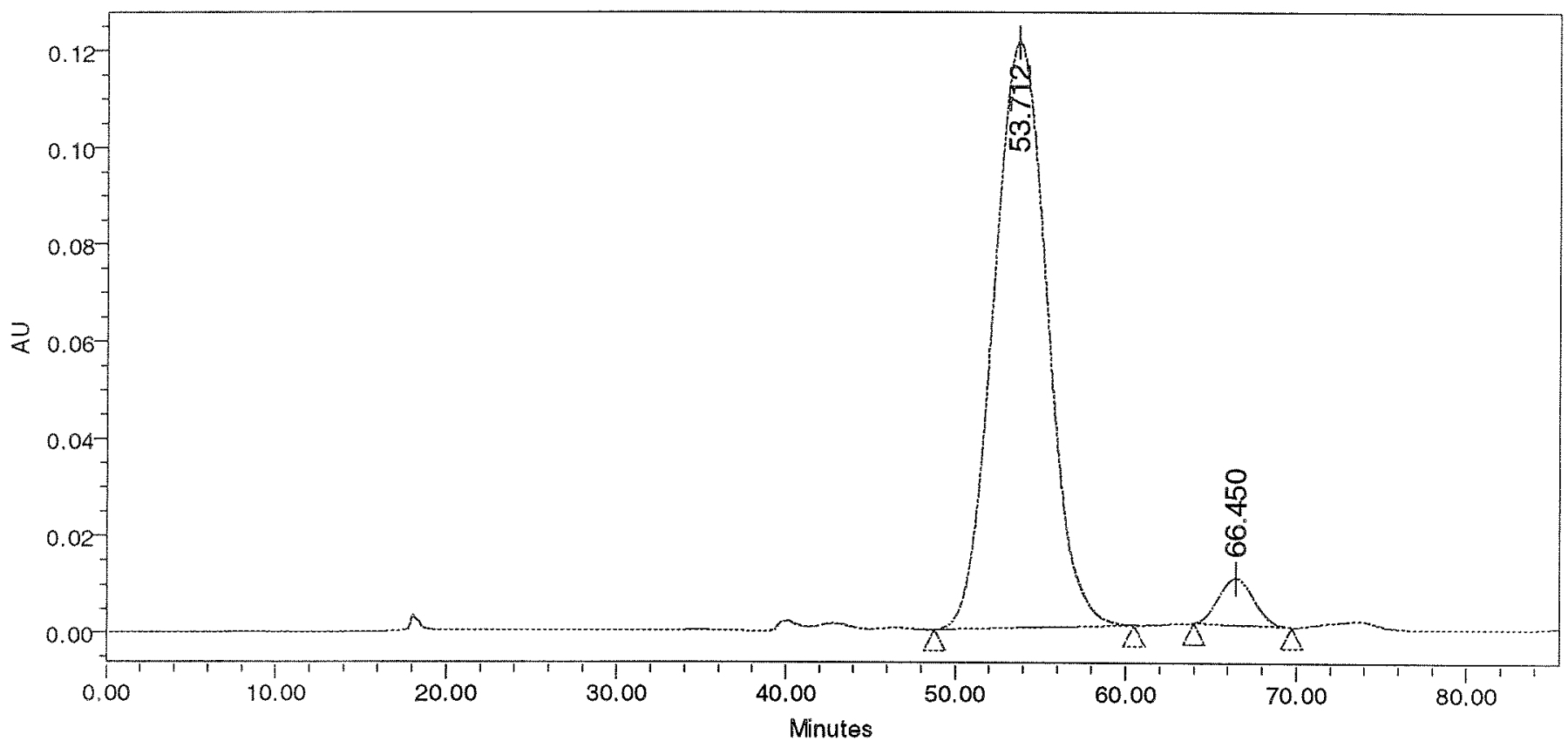

Peak Results

\begin{tabular}{|c|c|c|c|c|}
\hline & Name & RT & Area & $\%$ Area \\
\hline 1 & & 53.712 & 26446894 & 95.03 \\
\hline 2 & & 66.450 & 1383773 & 4.97 \\
\hline
\end{tabular}

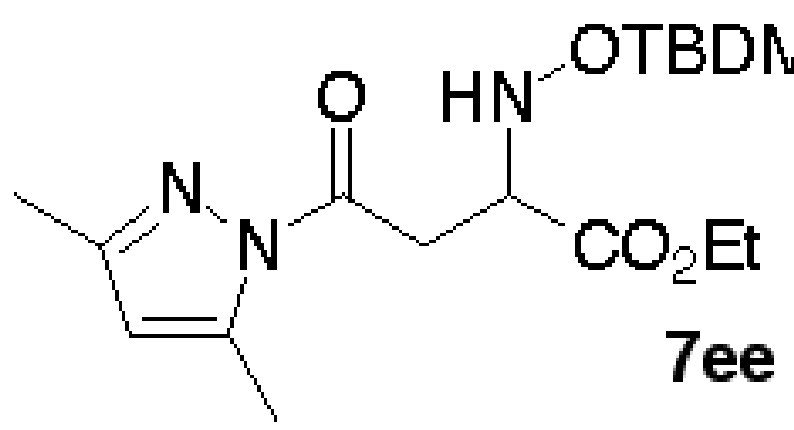




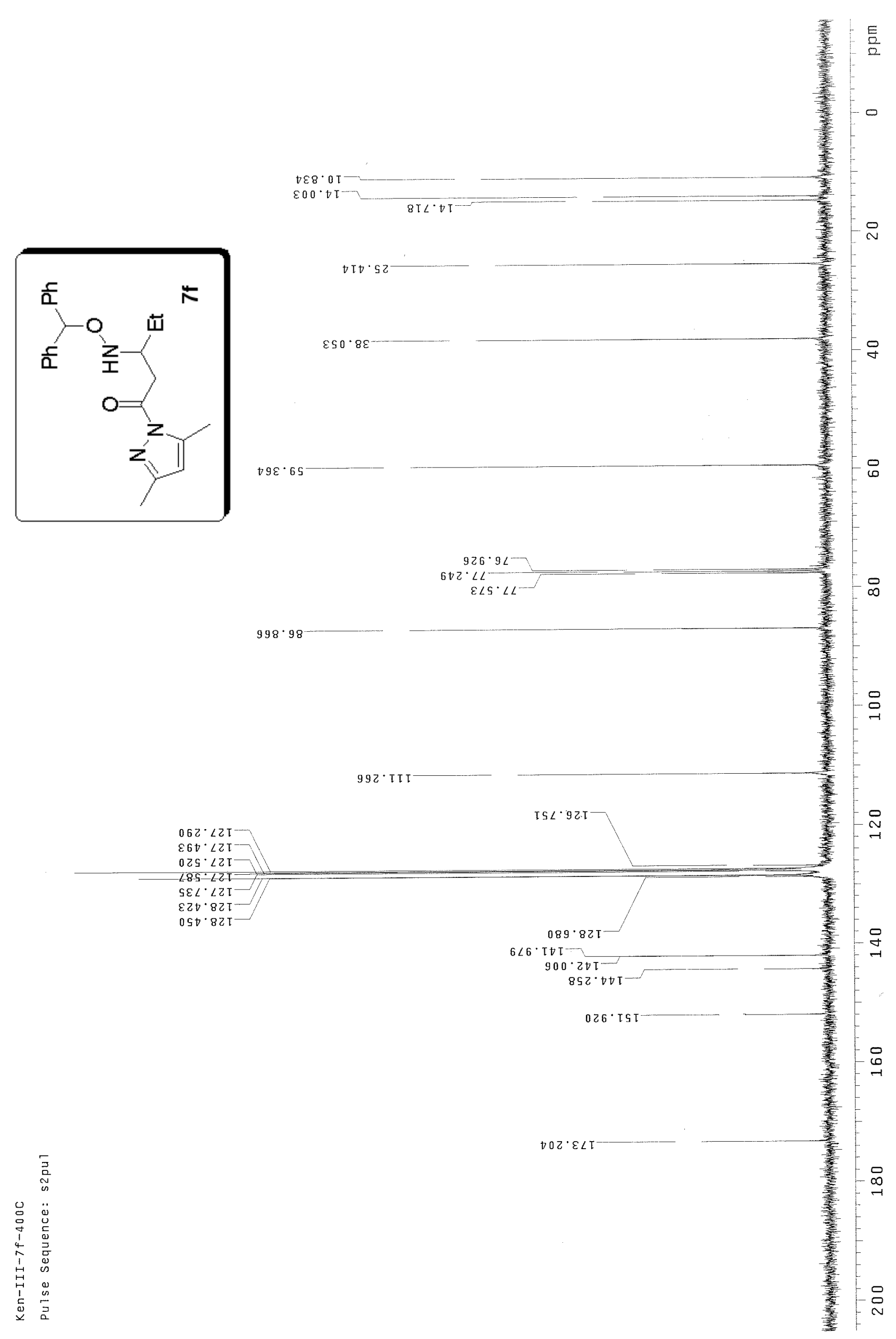




\section{Sample Information}

$\begin{array}{llll}\text { SampleName } & \text { KIIII831ADHp5\%1m } & \text { Sample Type } & \text { Unknown } \\ \text { Vial } & 1 & \text { Date Acquired } & \text { 2/7/2005 10:26:49 AM } \\ \text { Injection } & 1 & \text { Acq Method Set } & 5 \% \text { iPrOH 1mpm } \\ \text { Injection Volume } & 15.00 \mathrm{ul} & \text { Processing Method KIIII831ADHp5\%1m } \\ \text { Channel } & \text { 2487Channel 1 } & \text { Date Processed } & \text { 2/7/2005 11:40:33 AM } \\ \text { Run Time } & \text { 240.0 Minutes } & \end{array}$

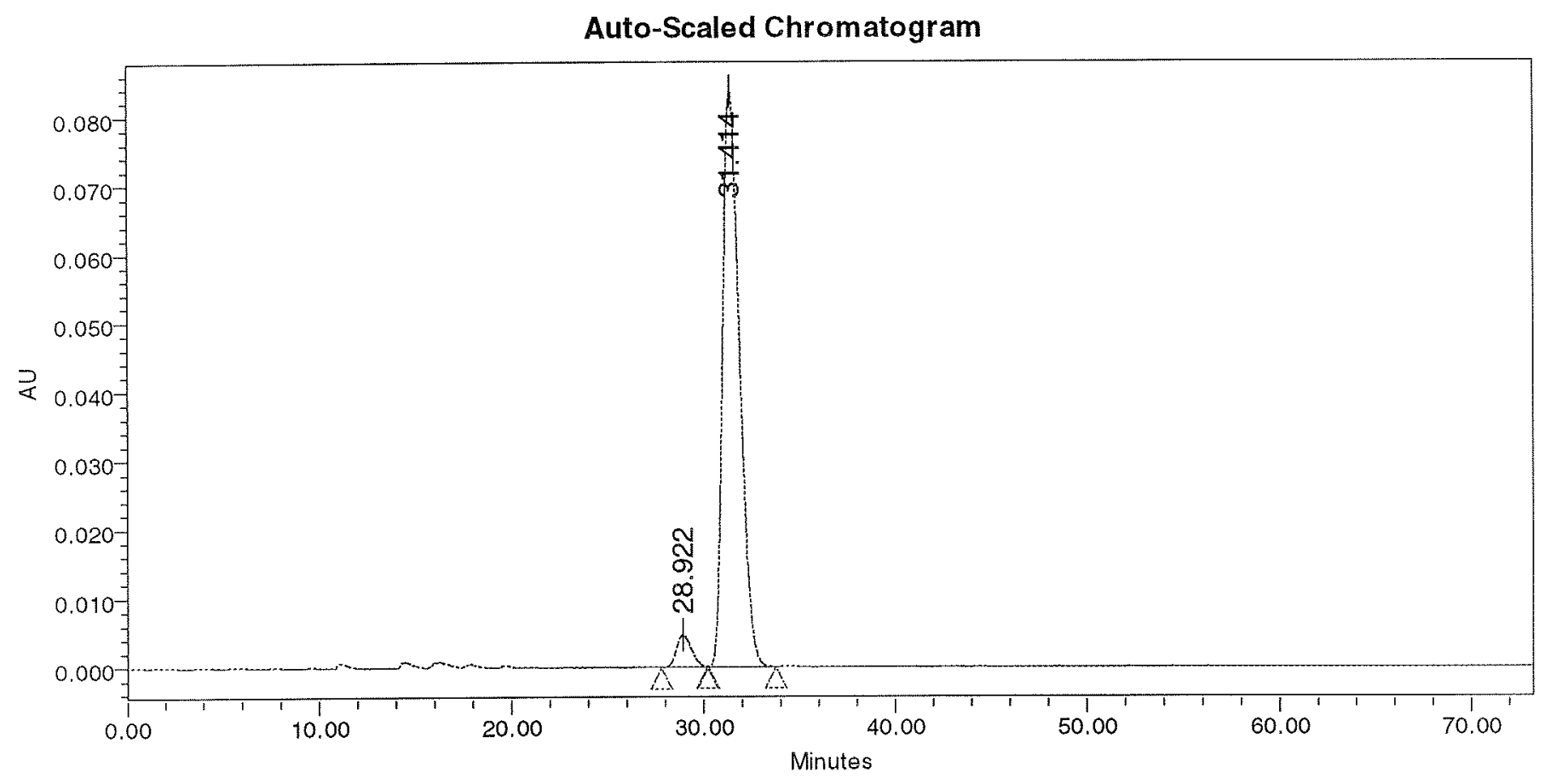

Peak Results

\begin{tabular}{|c|c|c|c|r|}
\hline & Name & RT & Area & $\%$ Area \\
\hline 1 & & 28.922 & 255137 & 4.76 \\
\hline 2 & & 31.414 & 5101015 & 95.24 \\
\hline
\end{tabular}

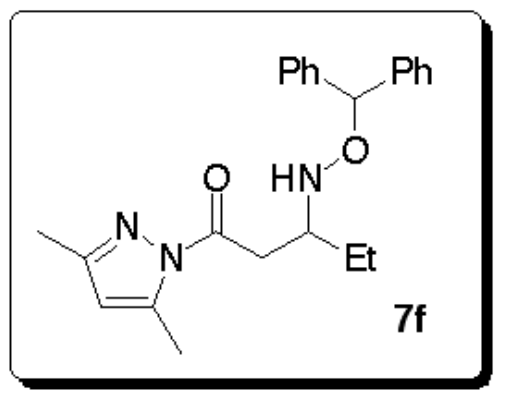


9

a)

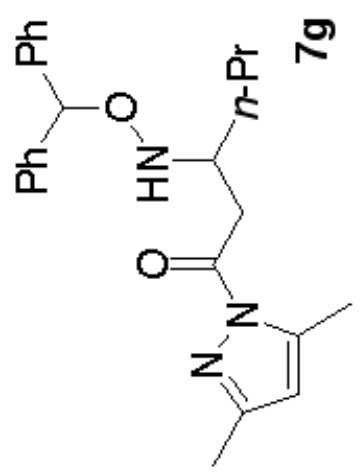

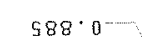

$806^{\circ}$

$\angle Z E^{\circ} T^{\circ}$

เซ $\cdot \mathrm{T}$

$05 \varepsilon^{\circ}$
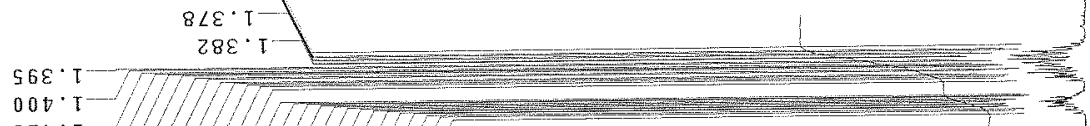

000

ETD'

$\varepsilon Z D^{\circ} T$

Get-T

sto
$095 \cdot-$

$890^{\circ} \mathrm{T}$

$2 \angle 0^{\circ} \mathrm{T}$

$280^{\circ} \mathrm{I}$

867

$855^{\circ}$
$695^{\circ}$

$895^{\circ} \mathrm{T}$

$S \angle S^{\circ} \mathrm{T}$

$185^{\circ} \mathrm{T}$

$\angle 85^{\circ} T$

$009^{\circ} T^{-}$

$709^{\circ} \mathrm{T}$

ZT9. T.

$\angle T \cdot T$

DE9 I

$800^{\circ} z$

$602^{\circ} z^{-}$

$81 \mathrm{t}^{\circ}$

$060^{\circ} \varepsilon$

$00 T^{\circ} \varepsilon$

$0 \varepsilon \mathrm{T} \cdot \varepsilon$

ot ${ }^{\circ} \varepsilon$

$9 \varepsilon \downarrow \cdot \varepsilon$

$\angle S D^{\circ} \varepsilon$

$9 \angle \mathrm{D} \cdot \mathrm{E}$

$96 t^{\circ} \varepsilon^{-}$

डा ${ }^{\circ} \varepsilon$

s2s. $\varepsilon$

$0 \varepsilon s^{*} \varepsilon^{\prime}$

$9 \varepsilon S^{\circ} \varepsilon$

$0 \nabla s \cdot \varepsilon$

sts $\varepsilon$

os $\varepsilon^{-}$

$\angle 55^{\circ} \varepsilon$

$\varepsilon 9 S^{\circ} \varepsilon$

$\angle \angle S^{\circ} \varepsilon^{\circ}$

०ह9.

218.5

$128 \cdot 5$

$5 \angle 1^{\circ} L^{-}$

$6 \angle \mathrm{T}^{\circ} \angle$

$98 \mathrm{I}^{\circ} \mathrm{L}$

$68 \mathrm{~T}^{\circ} \mathrm{L}^{-}$

$96 \mathrm{~T}^{\circ} \mathrm{L}$

$\operatorname{tod} L$

$\triangle T Z^{\circ} L$

$8 T 2 \cdot \angle$

$\mathrm{s} 2 \mathrm{C}^{\circ} \angle$

$9 \varepsilon Z \cdot \angle$

$202 \cdot L^{-}$

$\angle D Z Z^{\circ} \angle$

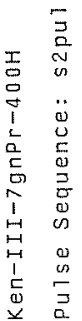

$658^{\circ} 0^{-}$

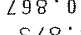

$\angle 9 \varepsilon$.

$8 \angle \varepsilon^{\circ} \tau$

틀

$N$

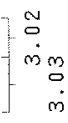

$m$

$\mid \begin{aligned} & -1 \\ & \infty \\ & 0 \\ & 0 \\ & 0 \\ & -1\end{aligned}$

$\checkmark$

in

$0 \int_{-1}^{-9}$

$-N$

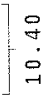

$-\infty$

$\sigma$

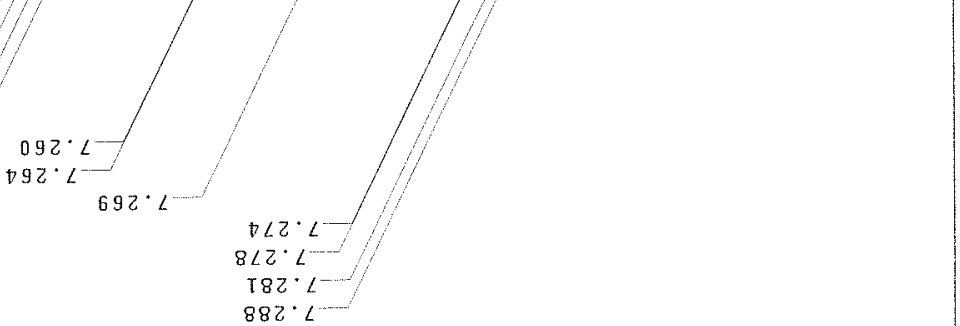

車 


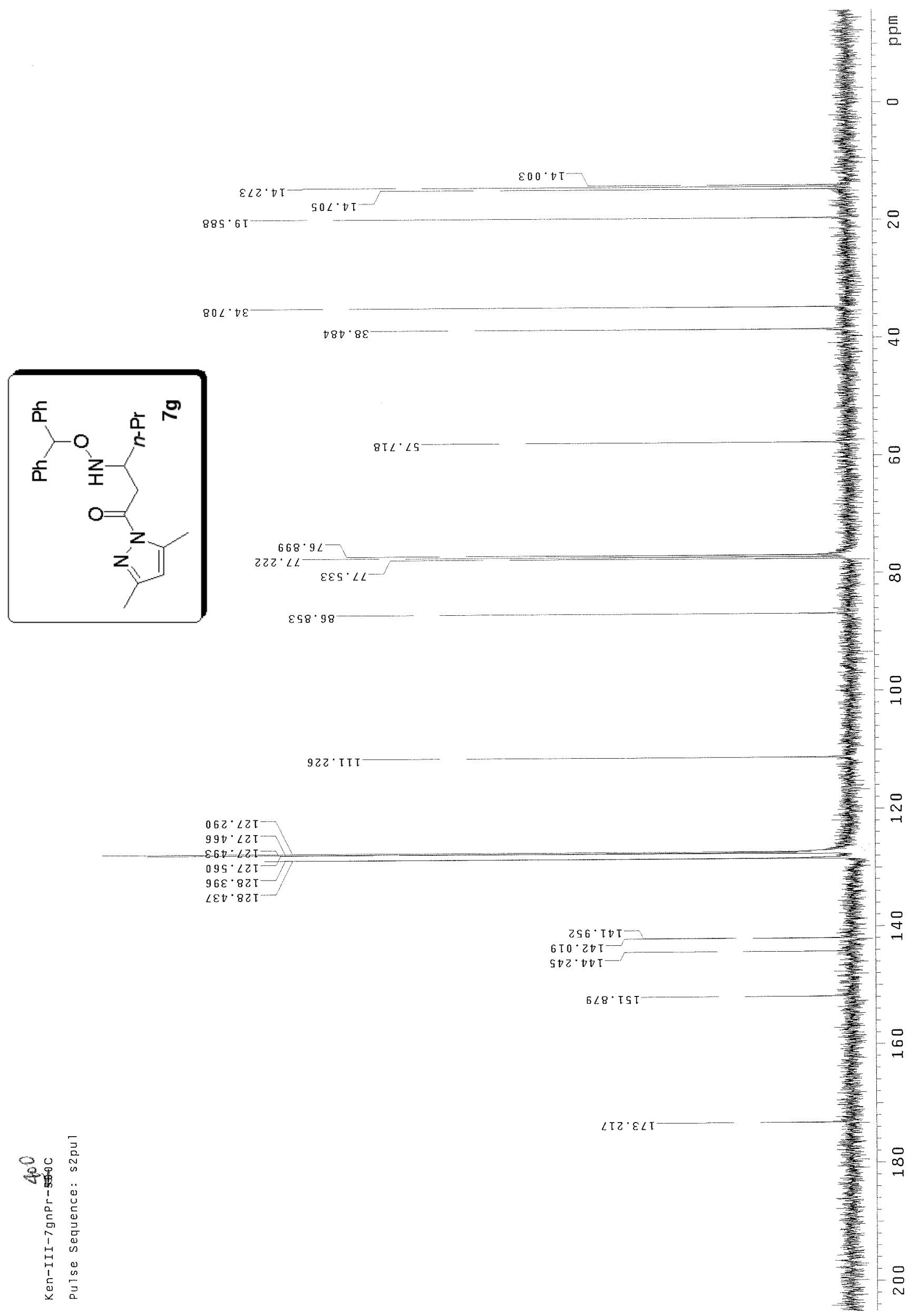




\section{Sample Information}

SampleName KIIII832ADHp5\%1ml

Vial 1

Injection

Injection Volume

Channel

Run Time
$15.00 \mathrm{ul}$

2487Channel 1

240.0 Minutes
Sample Type Unknown

Date Acquired $\quad$ 2/7/2005 11:44:33 AM

Acq Method Set $5 \%$ iPrOH 1 mpm

Processing Method Klll1832ADHp5\%1m

Date Processed 2/7/2005 12:31:54 PM

Auto-Scaled Chromatogram

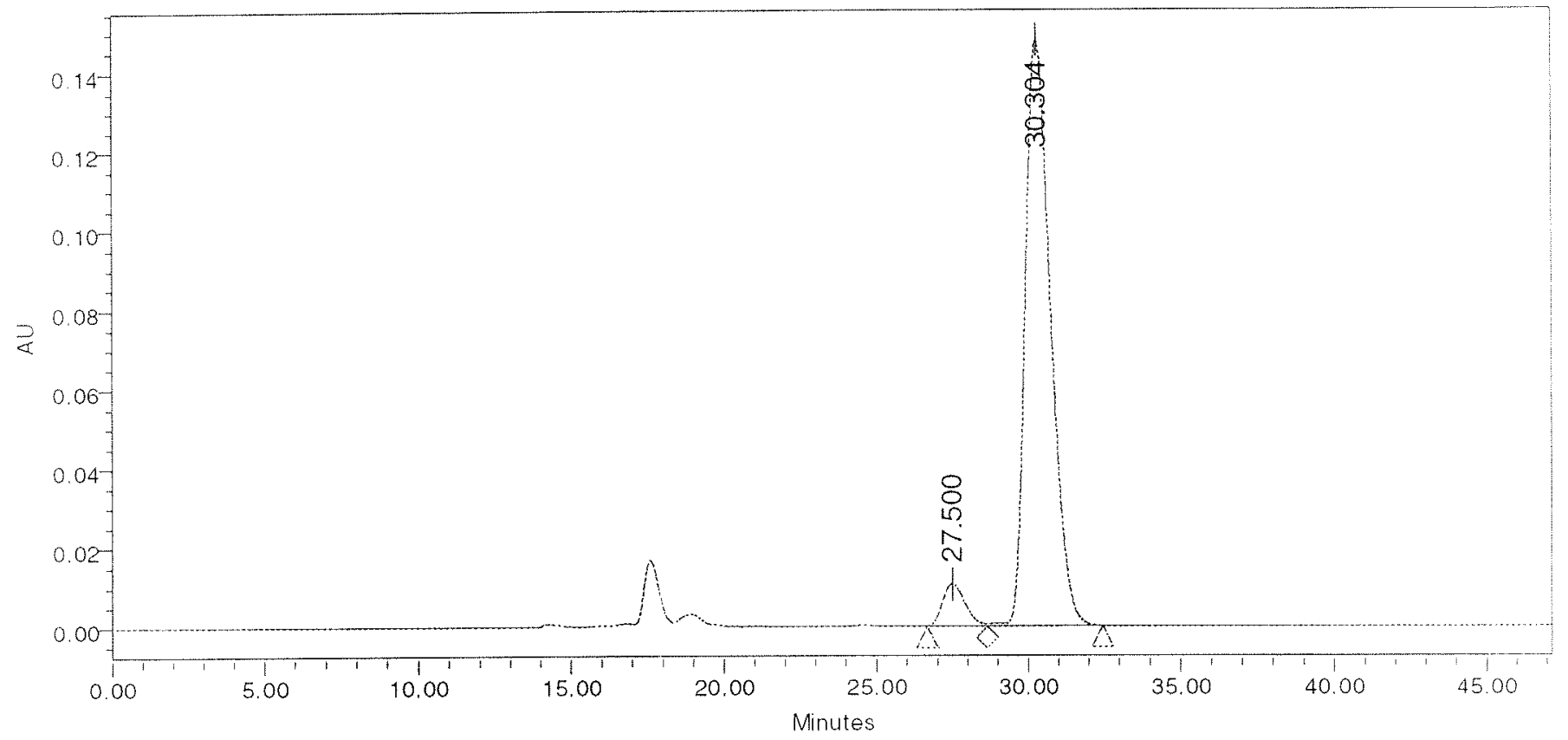

Peak Results

\begin{tabular}{|r|c|c|r|r|}
\hline & Name & RT & Area & $\%$ Area \\
\hline 1 & & 27.500 & 549149 & 6.07 \\
\hline 2 & & 30.304 & 8500766 & 93.93 \\
\hline
\end{tabular}

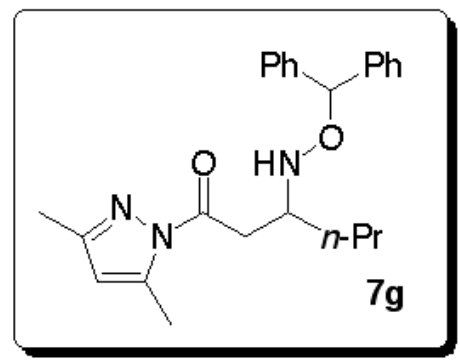



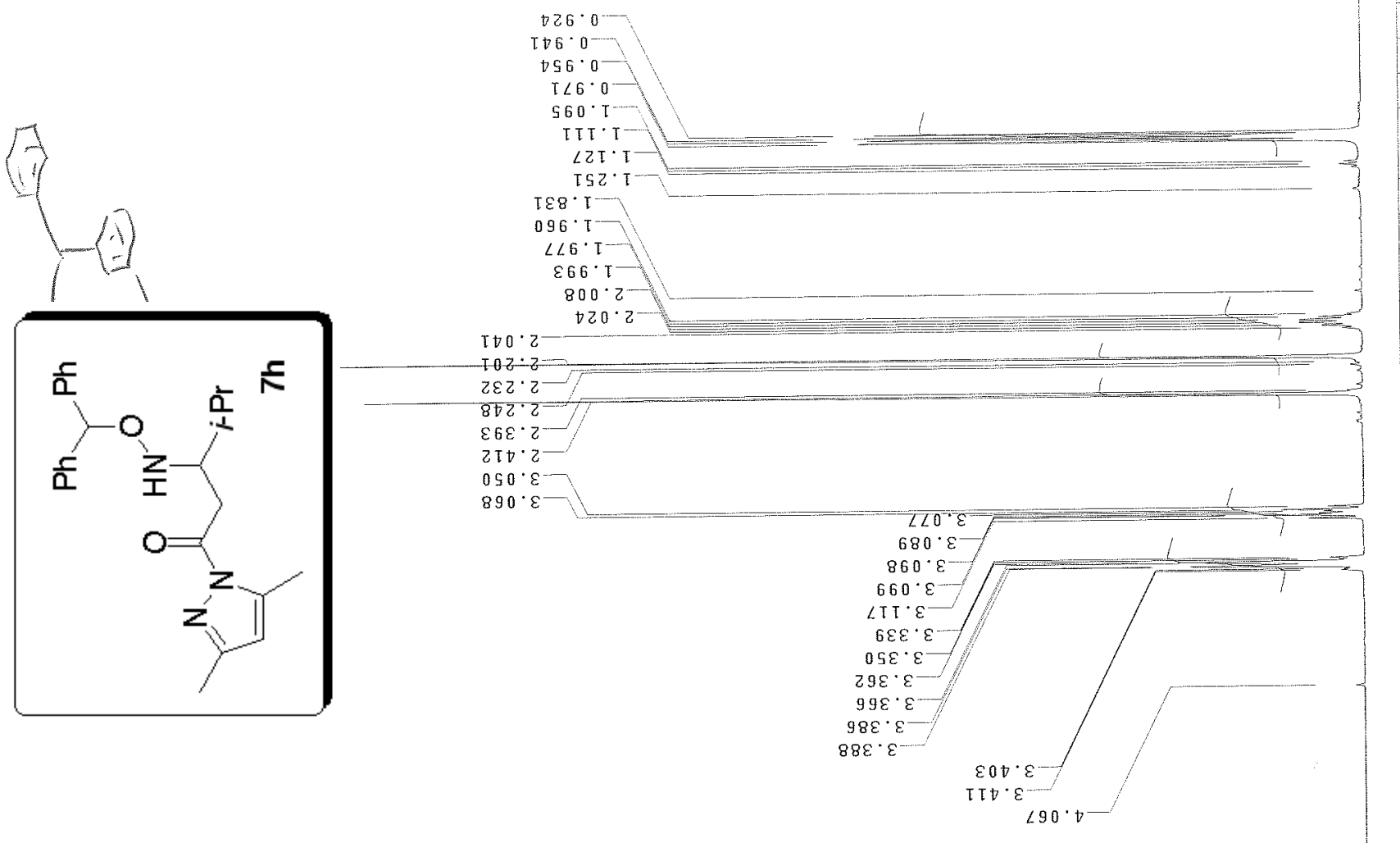

$-1 \stackrel{\infty}{N}$

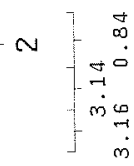

$m$

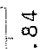
0
0
0
0
$n$

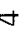

ก

0
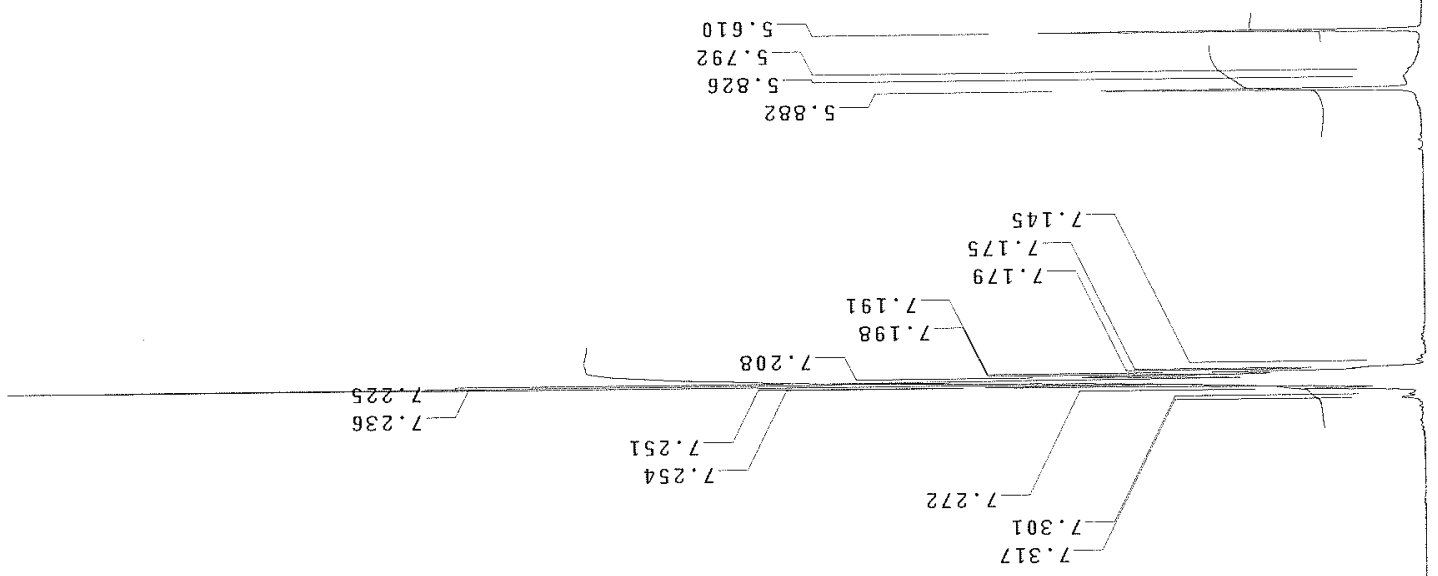

$288 \cdot 5$

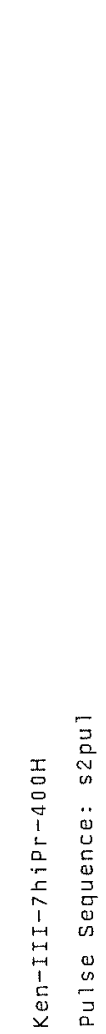

$\angle T E \cdot \angle$

$\infty$

$\pi$ 


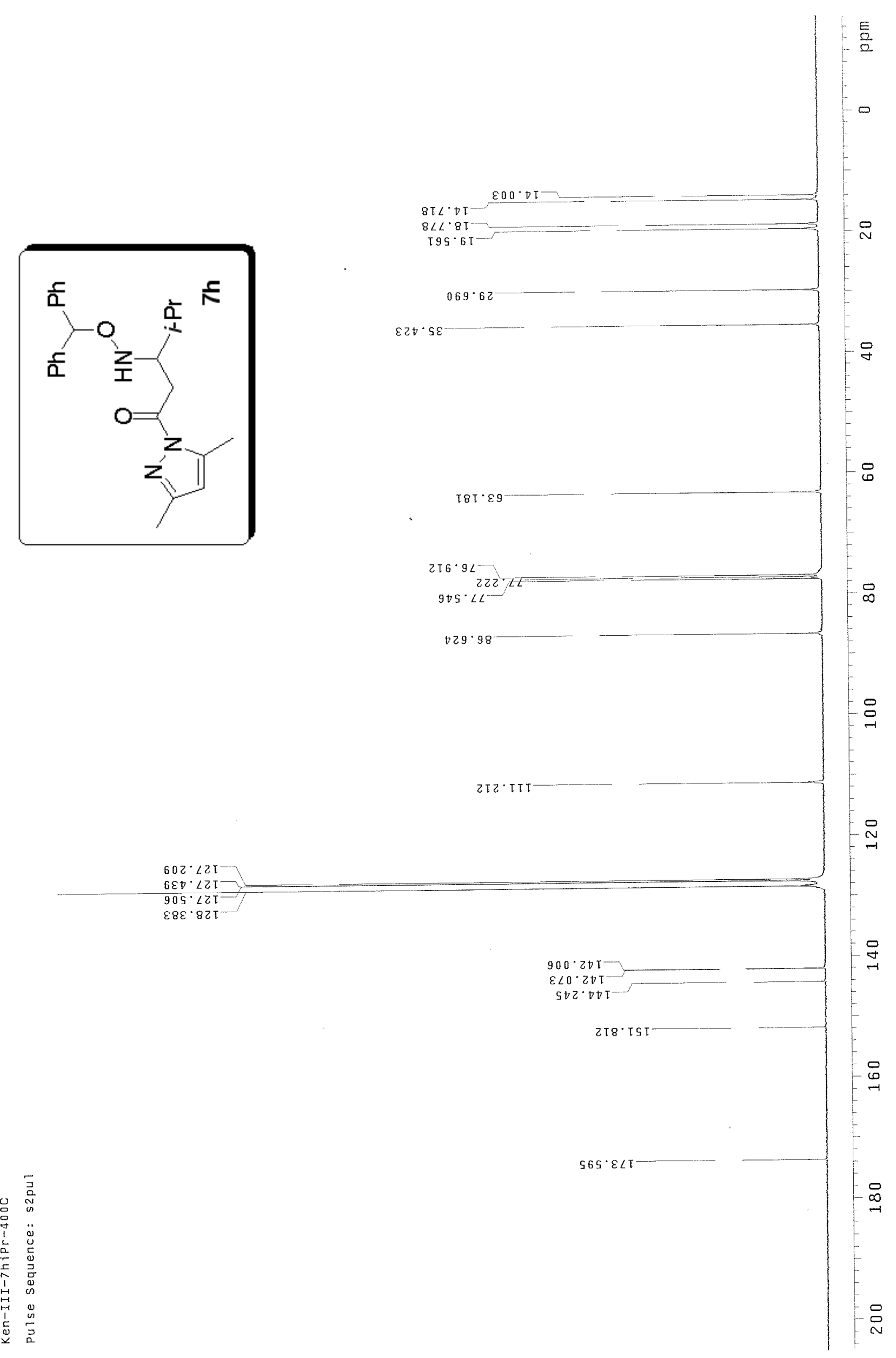




\section{Sample Information}

$\begin{array}{ll}\text { SampleName } & \text { KIII833ADHp5\%1m } \\ \text { Vial } & 1 \\ \text { Injection } & 3 \\ \text { Injection Volume } & 15.00 \text { ul } \\ \text { Channel } & 2487 \text { Channel } 1 \\ \text { Run Time } & 240.0 \text { Minutes }\end{array}$

Sample Type Unknown

Date Acquired $\quad$ 2/7/2005 12:36:19 PM

Acq Method Set $5 \% \mathrm{PrOH} 1 \mathrm{mpm}$

Processing Method KIIII833ADHp5\%1m

Date Processed 2/7/2005 1:56:48 PM

Run Time $\quad 240.0$ Minutes

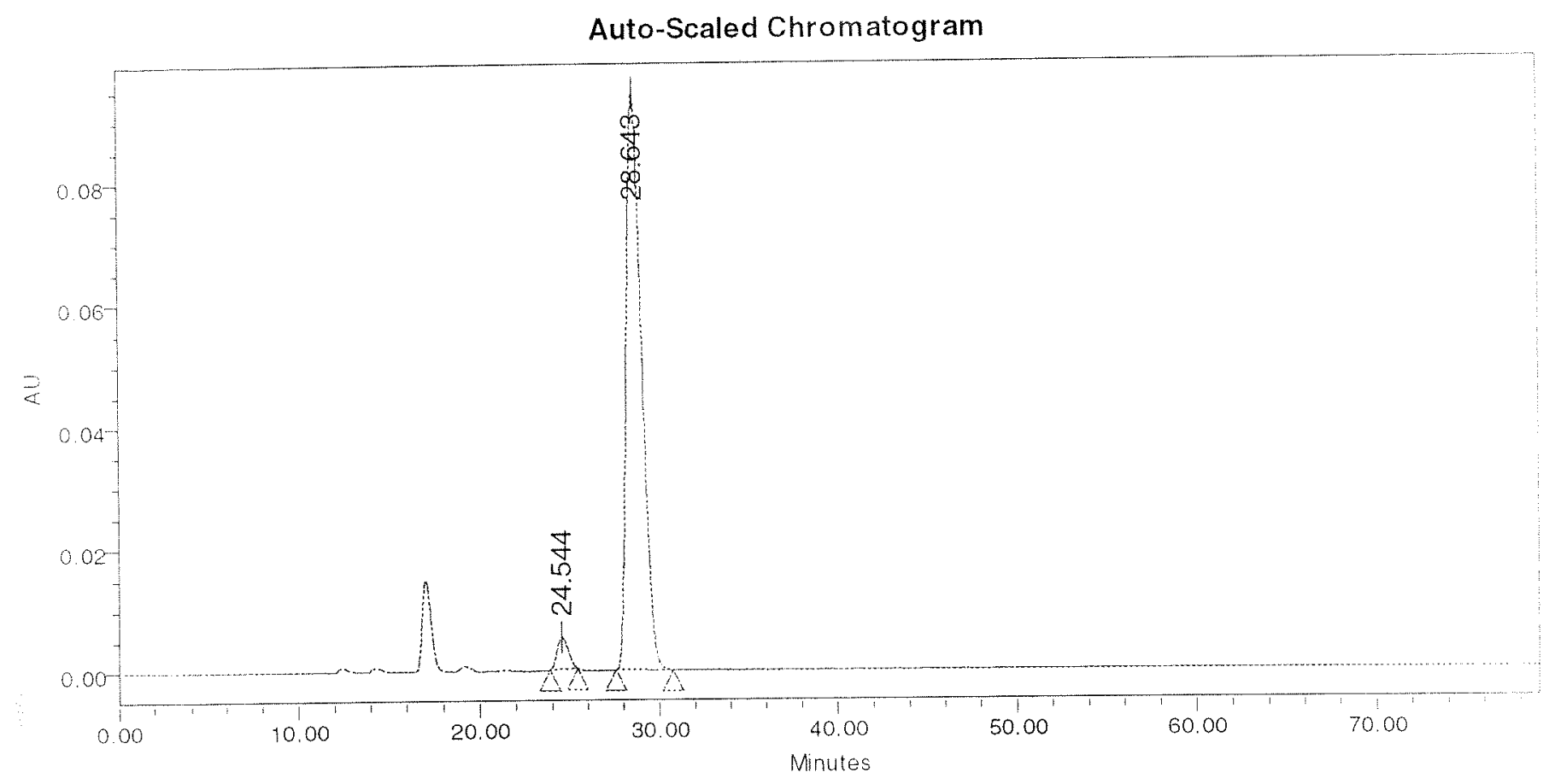

\begin{tabular}{|r|c|c|c|r|}
\multicolumn{5}{|c|}{ Peak Results } \\
\hline & Name & RT & Area & $\%$ Area \\
\hline 1 & & 24.544 & 230988 & 3.95 \\
\hline 2 & & 28.643 & 5611272 & 96.05 \\
\hline
\end{tabular}

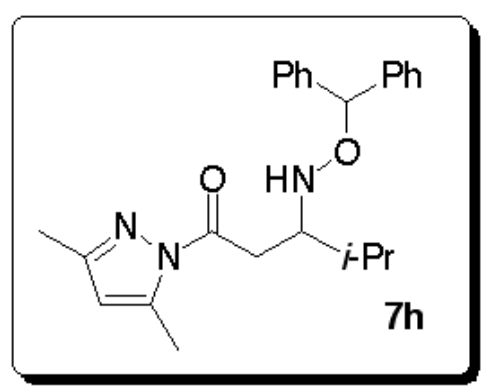


$\sqrt{11}$

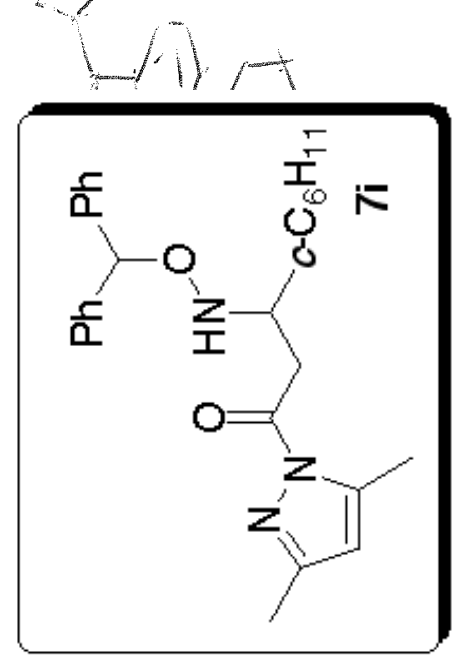

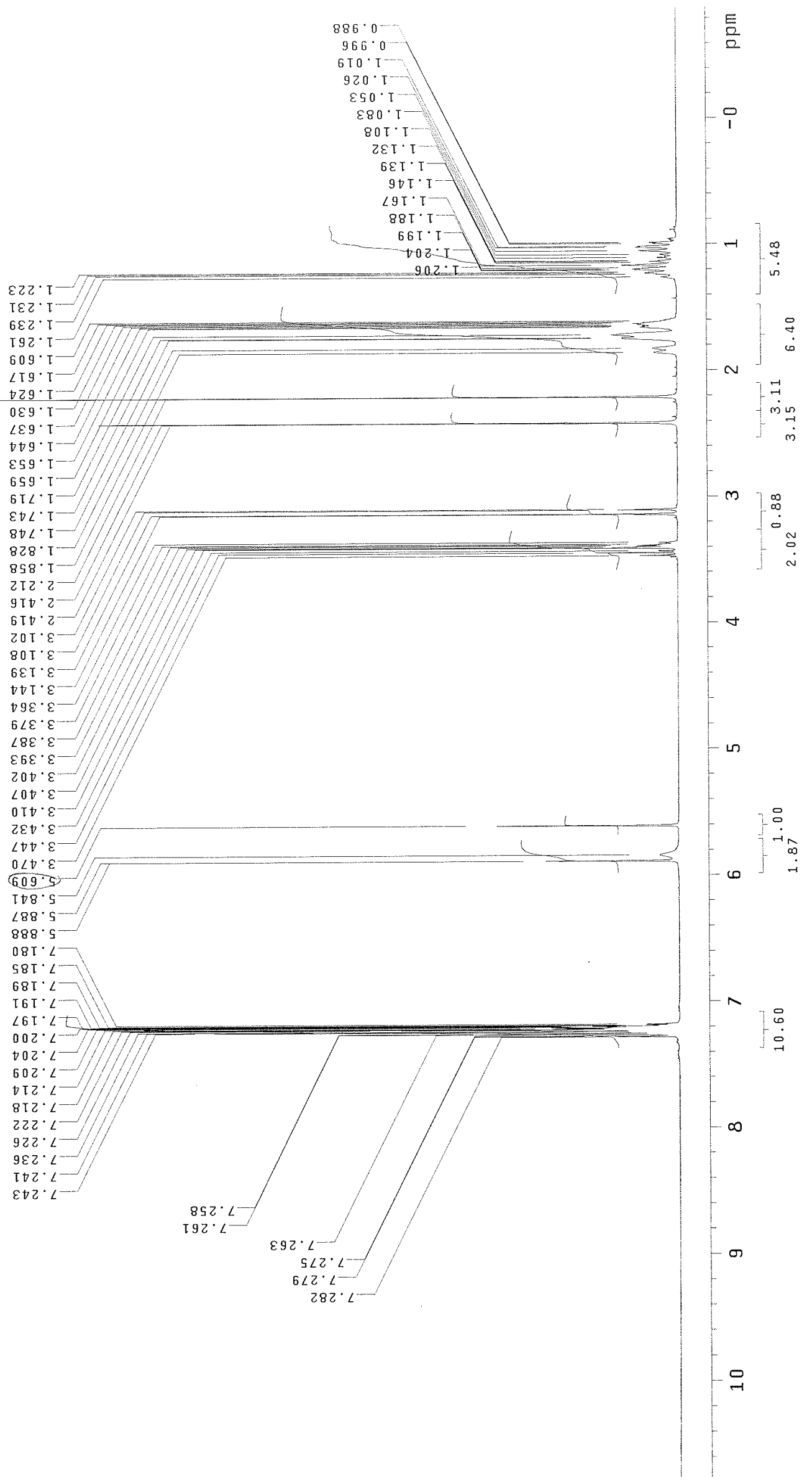




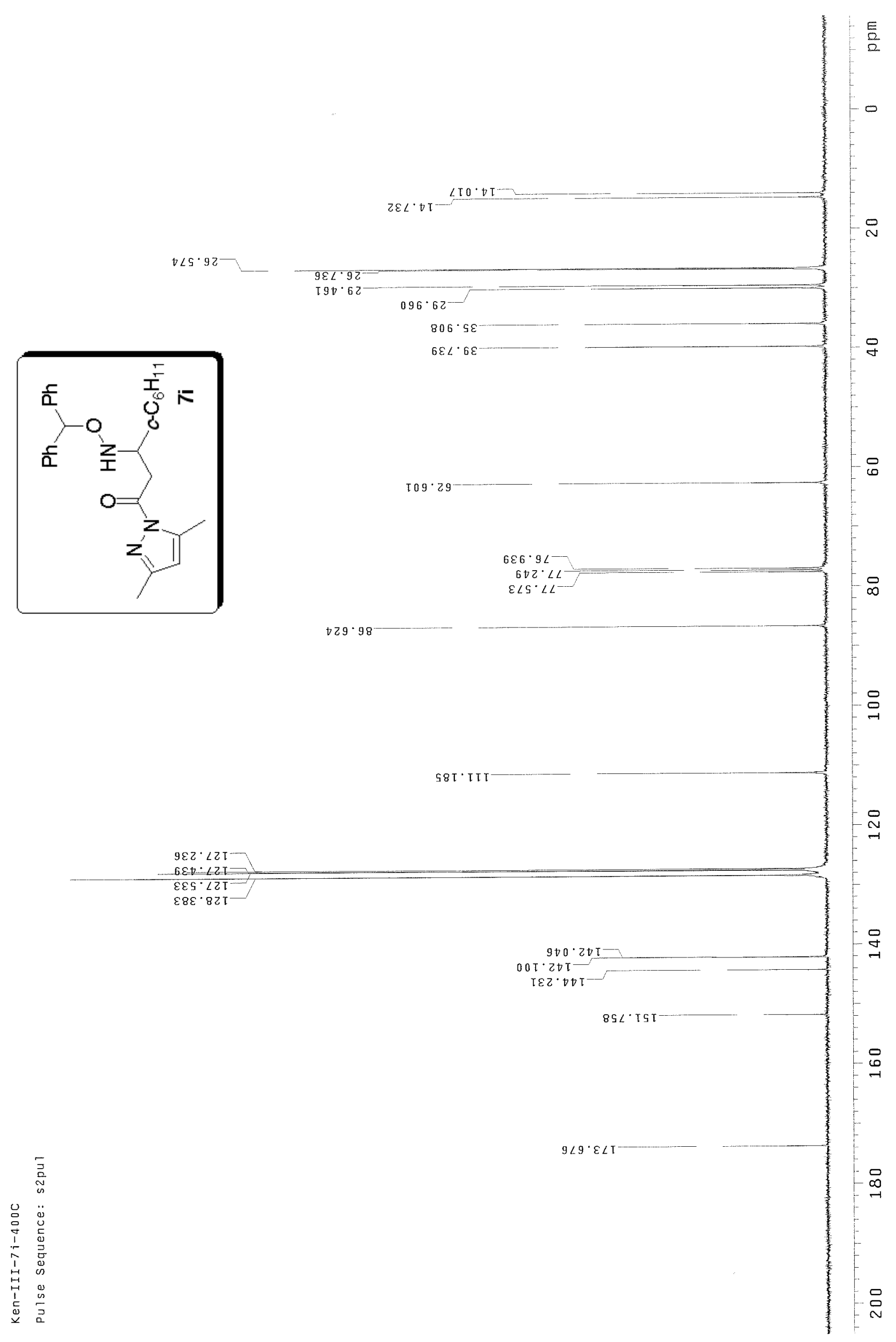




\section{Sample Information}

$\begin{array}{ll}\text { SampleName } & \text { KIlll834ADHp5\%1m } \\ \text { Vial } & 1 \\ \text { Injection } & 4 \\ \text { Injection Volume } & 15.00 \mathrm{ul} \\ \text { Channel } & \text { 2487Channel 1 } \\ \text { Run Time } & \text { 240.0 Minutes }\end{array}$

Sample Type Unknown

Date Acquired $\quad$ 2/7/2005 2:00:12 PM

Acq Method Set $5 \%$ iPrOH $1 \mathrm{mpm}$

Processing Method KIIII834ADHp5\%1m

Date Processed ～2/7/2005 2:56:37 PM

Run Time 240.0 Minutes

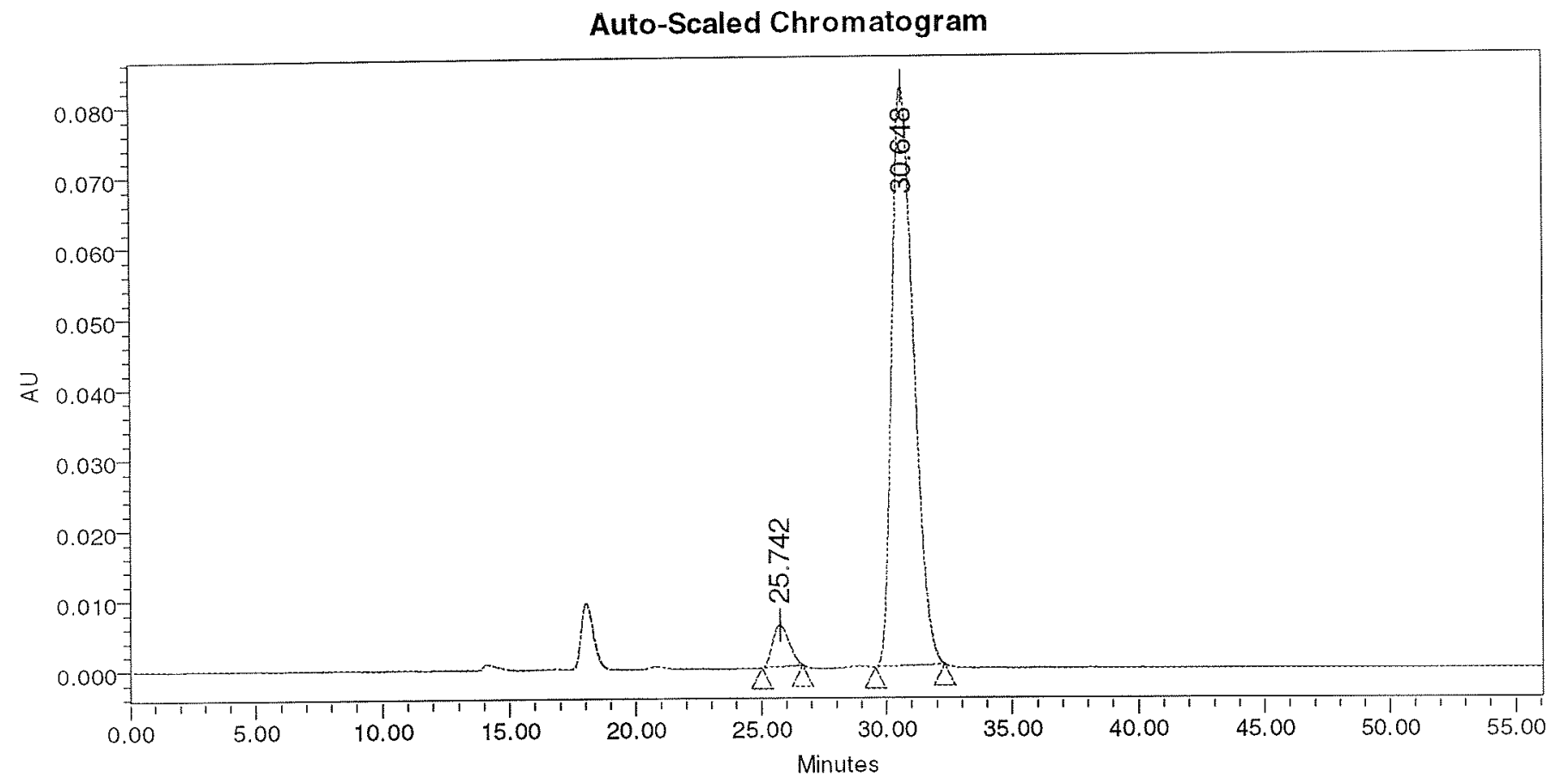

Peak Results

\begin{tabular}{|l|c|c|c|r|}
\hline & Name & RT & Area & $\%$ Area \\
\hline 1 & & 25.742 & 271813 & 5.25 \\
\hline 2 & & 30.648 & 4904586 & 94.75 \\
\hline
\end{tabular}

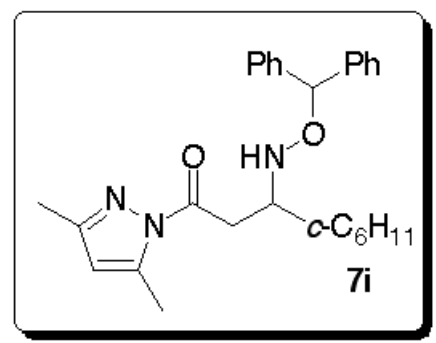




\section{Sample Information}

$\begin{array}{llll}\text { SampleName } & \text { KIIII789ADH20\%1m } & \text { Sample Type } & \text { Unknown } \\ \text { Vial } & 1 & \text { Date Acquired } & \text { 1/3/2005 8:48:04 PM } \\ \text { Injection } & 1 & \text { Acq Method Set } & \text { 20\%iPrOH 1mpm } \\ \text { Injection Volume } & 20.00 \mathrm{ul} & \text { Processing Method KIII789ADH20\%1m } \\ \text { Channel } & 2487 \text { Channel 1 } & \text { Date Processed } & \text { 1/3/2005 10:17:55 PM } \\ \text { Run Time } & \text { 240.0 Minutes } & \end{array}$

Auto-Scaled Chromatogram

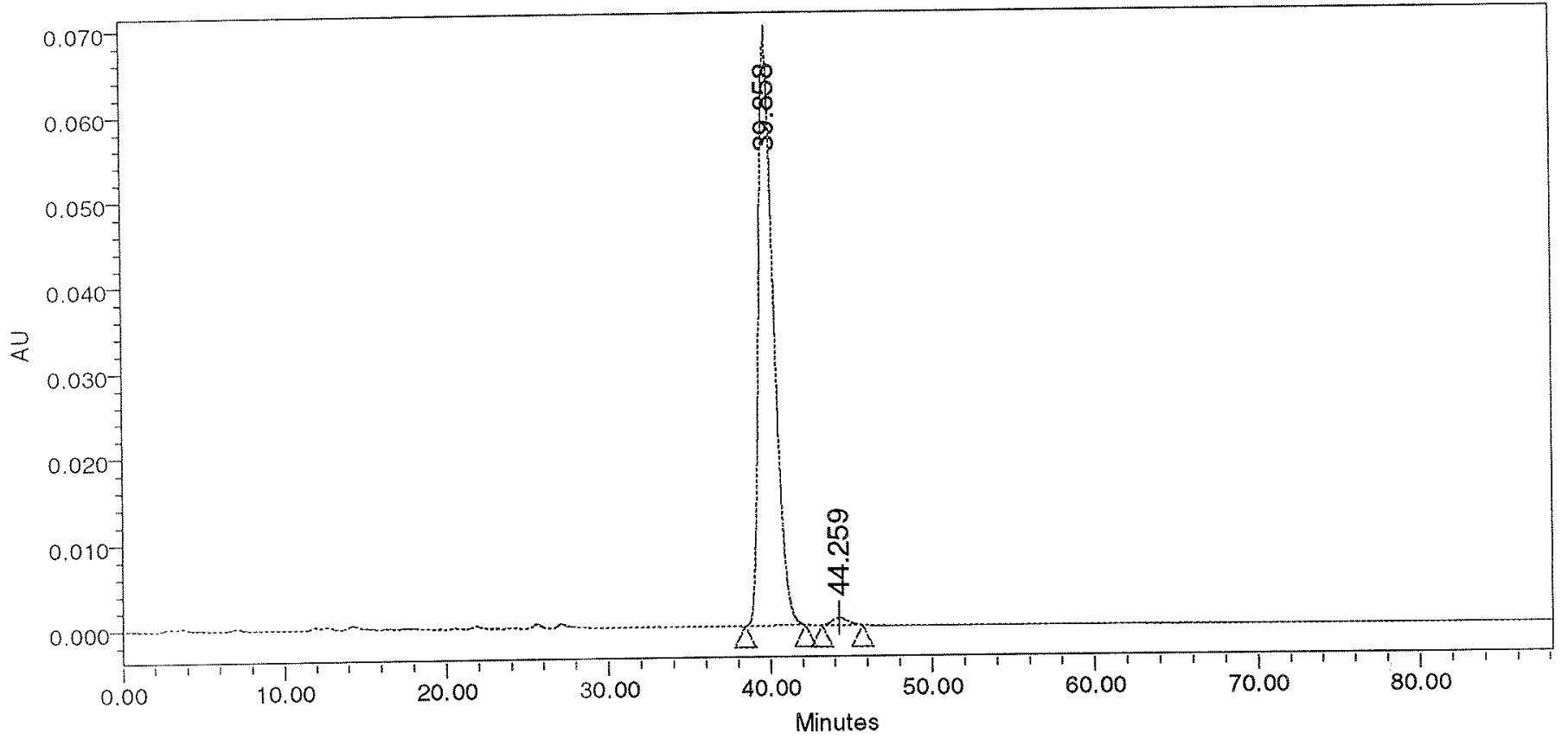

Peak Results

\begin{tabular}{|c|c|c|c|r|}
\hline & Name & RT & Area & $\%$ Area \\
\hline 1 & & 39.858 & 4497386 & 98.72 \\
\hline 2 & & 44.259 & 58323 & 1.28 \\
\hline
\end{tabular}

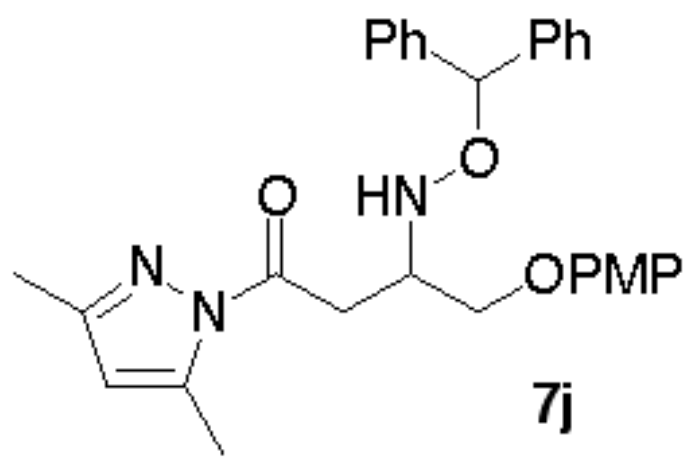




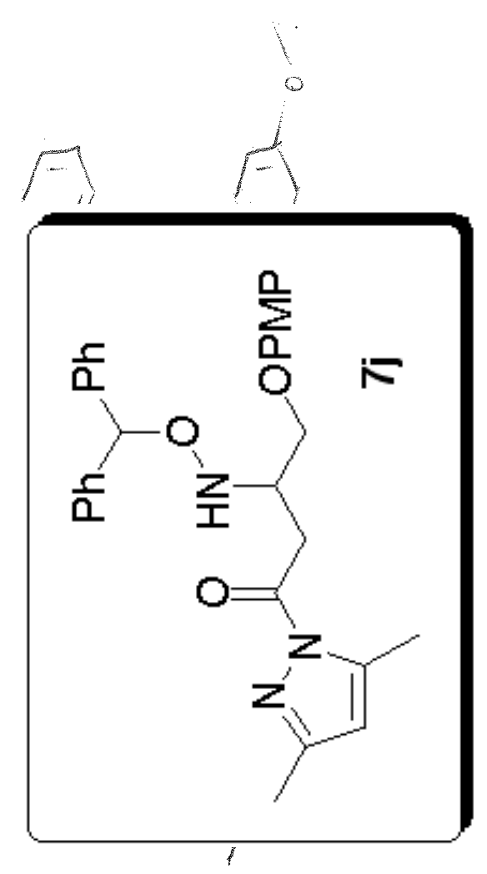

틀

$6.12 \cdot 2$

$\frac{612: 2}{980.2}$

$880^{\circ} \cdot$

$\varsigma \varepsilon \varepsilon \cdot \varepsilon$

$60 \varepsilon \cdot \varepsilon^{\circ}$

$8 \angle \varepsilon^{\circ} \varepsilon^{-}$

โ $6 \varepsilon \cdot \varepsilon$

$\nabla \varepsilon \varsigma^{\circ} \varepsilon$

$\varepsilon \varsigma \varsigma^{\prime} \varepsilon$

$9 / 5^{\circ} \varepsilon$

$565 \cdot 8$

$2 S \angle \varepsilon^{\circ}$

$85 \angle \circ$

$296 \cdot \varepsilon$

$\angle 96^{\circ} \cdot$
$T \angle 6^{\circ}$

$\mathrm{T} \angle 6^{\circ} \cdot \varepsilon^{\circ}$
$5 \angle 6$

पर००

$066^{\circ} \varepsilon$
$290^{\circ}$

$\checkmark \angle 0^{\circ} \circ$

$580^{\circ} \mathrm{b}$

$\angle 60^{\circ} \circ$

$80 T^{\circ}$

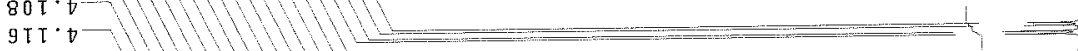

$\left.\varepsilon \tau^{\prime} \cdot \nabla^{-}\right)+\ldots$

T $\varepsilon$ T' $\cdot$ 十

$\varepsilon \mathcal{E} \cdot t=$

$90 \pi^{\circ} .00+2=$

$602.9 \mathrm{COUC=}=$

20.

826.9

$66 \angle \cdot 9$

โ08.9.

$808 \cdot 9$

Eโ8. 9

$\varepsilon 28.9$

$928 \cdot 9-$

$9 \varepsilon 8 \cdot 9$

TD $8 \cdot 9$

$808 \cdot 9$

$512 \cdot 2$

$812 \cdot 4$

$612 .<$

โ $22^{\circ}$

$\nabla z z \cdot \angle$

$822 \cdot 2$

$0 \varepsilon z \cdot\llcorner$

$\varepsilon \varepsilon \tau^{\circ} \cdot$

$s \varepsilon z \cdot L$

$8 \varepsilon z \cdot \angle$

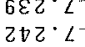

$202 \cdot 2$
$092 \cdot L$

TSZ $\angle$

$892^{\circ} /$

$992 \cdot L$

$892 \cdot 1$

$0 \angle 2 \cdot \angle$

$\angle \angle 2^{\circ} \angle$

$982^{\circ} \mathrm{L}$

$062 \%$

$\angle 62{ }^{\circ} \angle$ $i$

$-$

N

is

m

$\therefore$

-iक

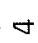

$\dot{m}$

J

$\dot{m}$

เ

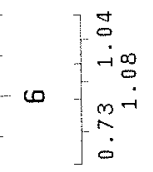

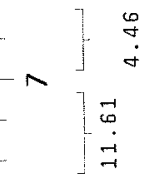

$\infty$

$a$

$80 \varepsilon: L$
$01 \varepsilon \angle$

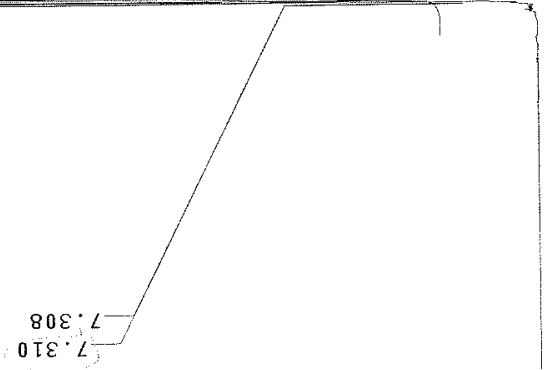

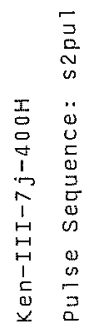

묵 

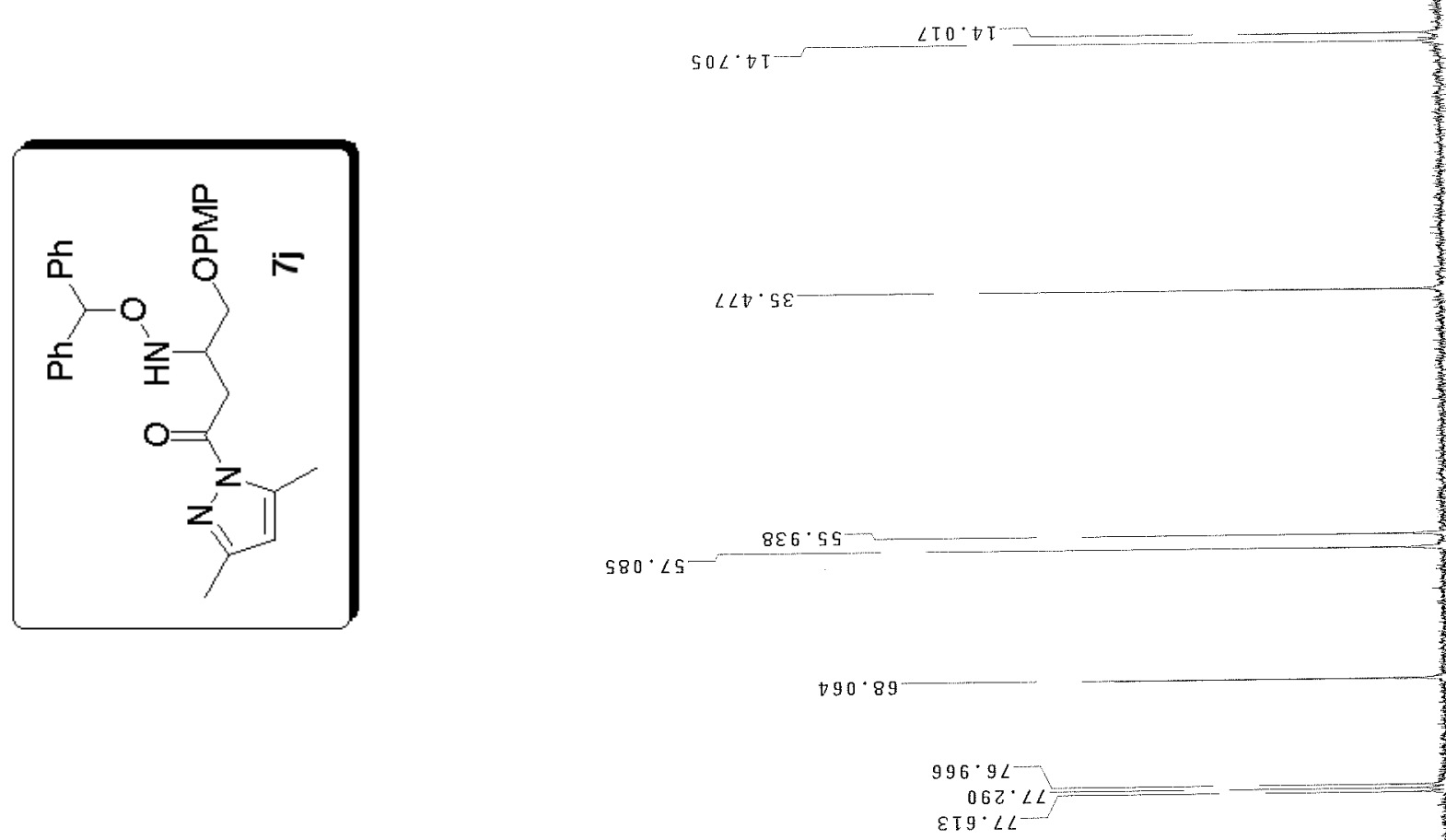

$270 \cdot \angle 8$

$88 \varepsilon^{\circ} \cdot I I$ $\frac{T \neq 8^{\circ}+t T}{\mathrm{SZ2} \cdot \mathrm{TT}}$

$86 \varepsilon^{\circ} \angle Z I$

$\varepsilon \varepsilon S^{\circ} \angle Z T-$

$189^{\circ} \angle 2 \mathrm{~T}$
$8 \mathrm{~T}$

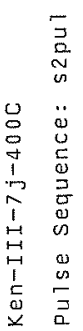

$\angle 2 \varepsilon \cdot 2 \angle r$

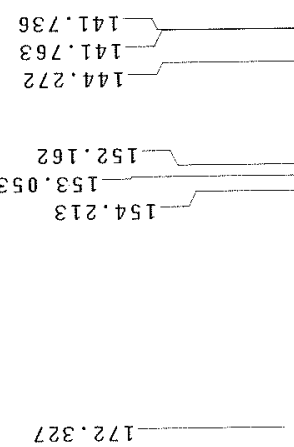

$\stackrel{0}{\stackrel{9}{+}}$

$\stackrel{2}{N}$ 

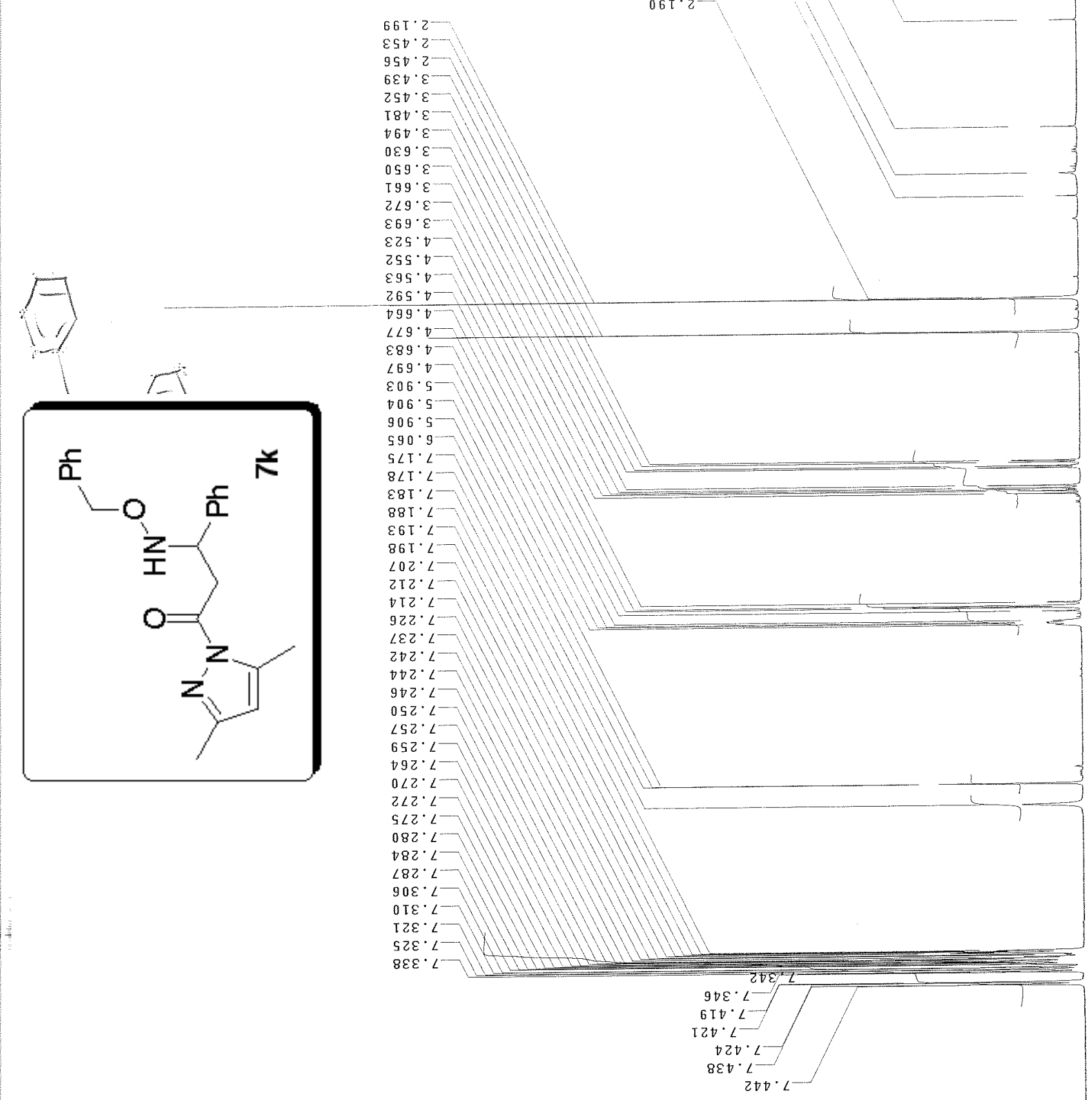

$N$

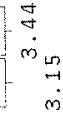

$m$

웅.

L

$\infty \frac{0}{0}$

$N$

$\therefore$

$\infty$ 

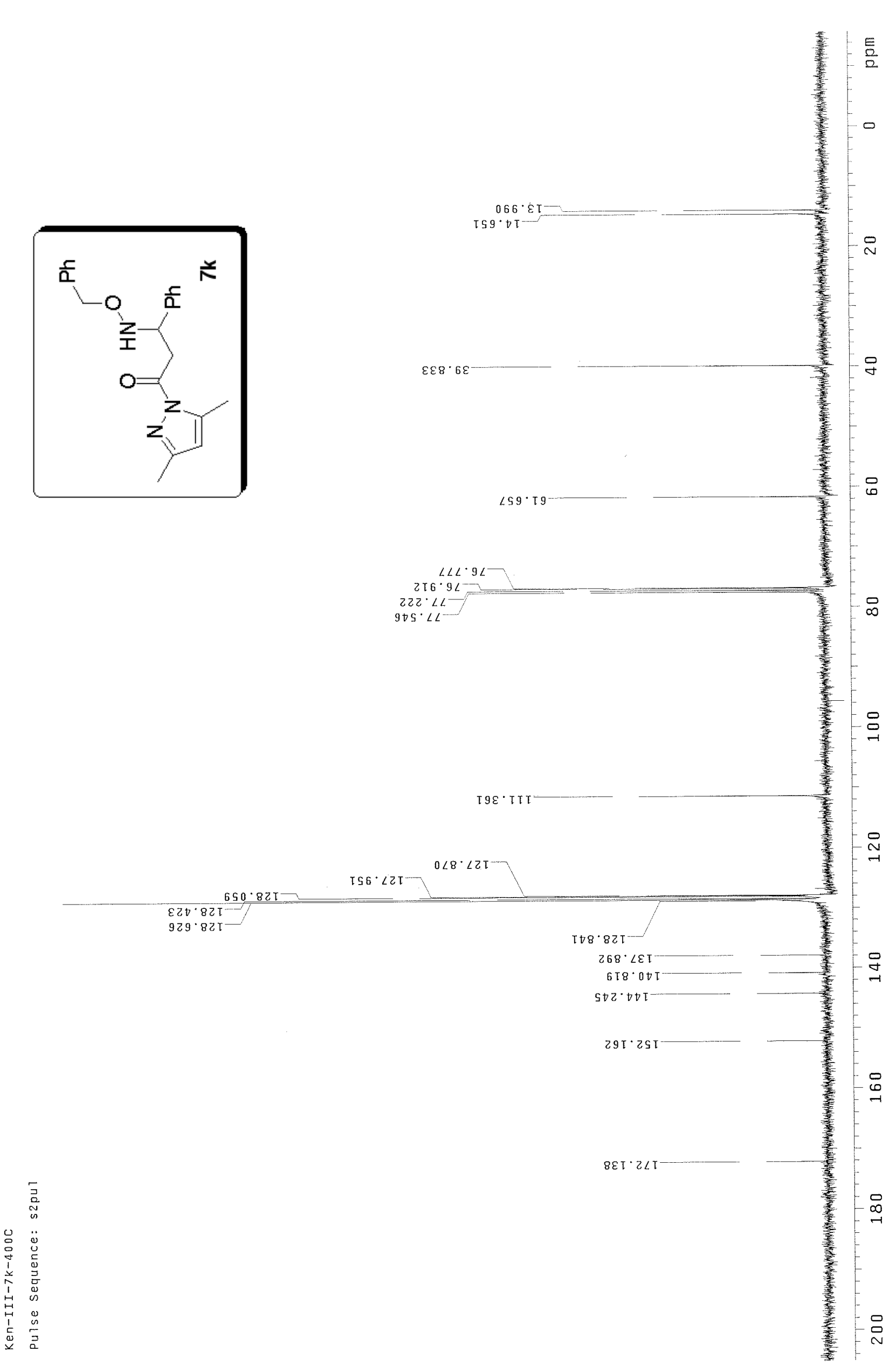


\section{Sample Information}

$\begin{array}{llll}\text { SampleName } & \text { Kll552OJ10\%1m } & \text { Sample Type } & \text { Unknown } \\ \text { Vial } & 1 & \text { Date Acquired } & \text { 9/8/2004 5:37:14 PM } \\ \text { Injection } & 2 & \text { Acq Method Set } & \text { 10\%iPrOH impm } \\ \text { Injection Volume } & 10.00 \mathrm{ul} & \text { Processing Method KIl552OJ10\%1m } \\ \text { Channel } & \text { 2487Channel 1 } & \text { Date Processed } & \text { 9/8/2004 6:29:41 PM } \\ \text { Run Time } & \text { 240.0 Minutes } & \end{array}$

Auto-Scaled Chromatogram

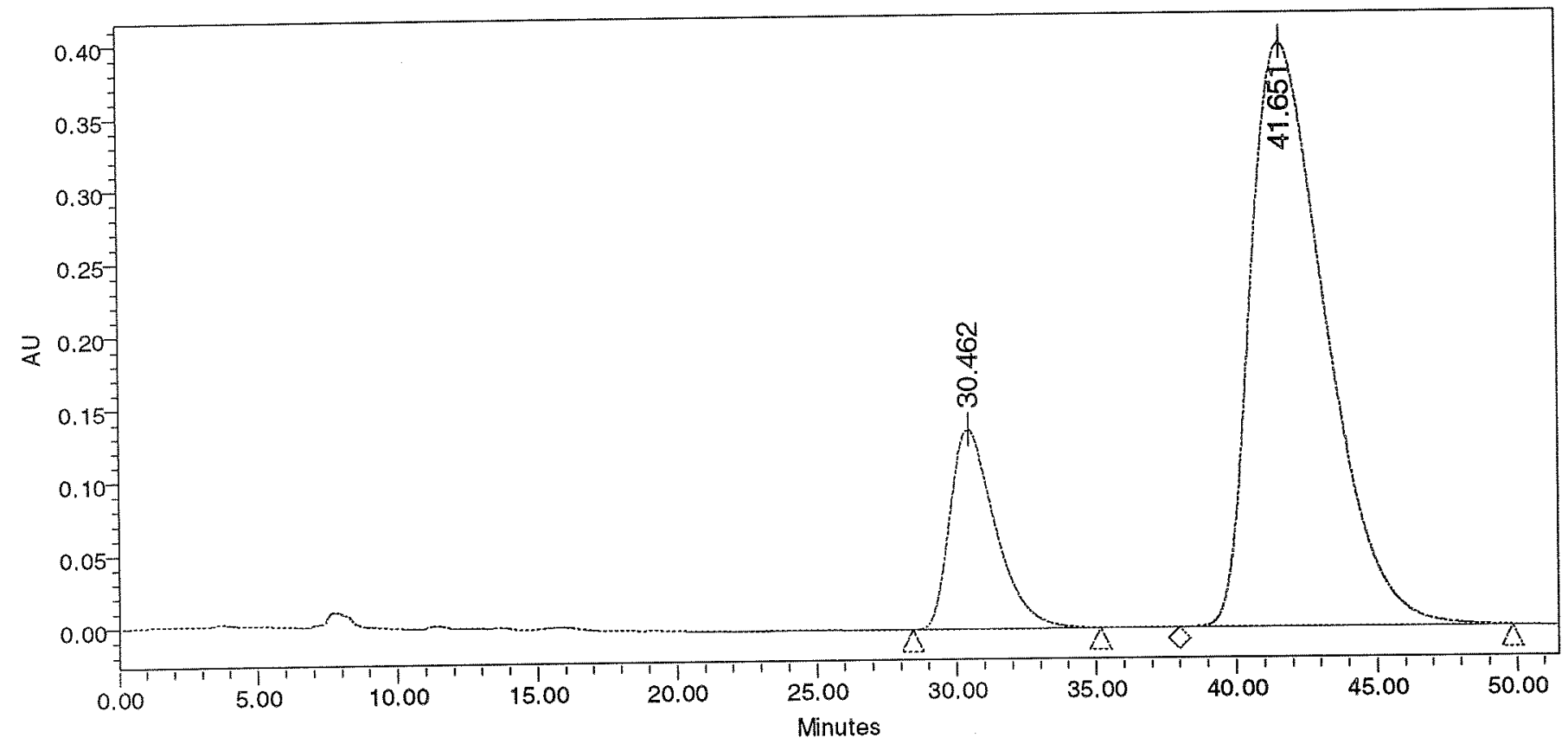

Peak Results

\begin{tabular}{|c|c|c|c|c|}
\hline & Name & RT & Area & $\%$ Area \\
\hline 1 & & 30.462 & 15492255 & 17.37 \\
\hline 2 & & 41.651 & 73675816 & 82.63 \\
\hline
\end{tabular}

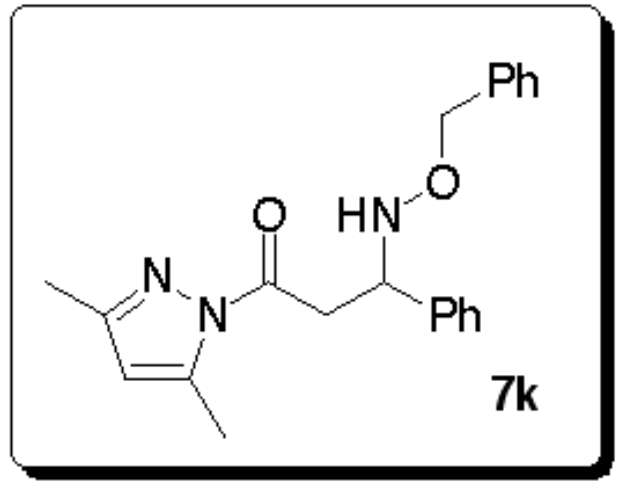

